九州大学学術情報リポジトリ

Kyushu University Institutional Repository

\title{
Unified approach to the classification of actions of discrete amenable groups on injective factors
}

Masuda, Toshihiko

Graduate School of Mathematics, Kyushu University

http://hdl. handle. net/2324/4485865

出版情報: Journal für die reine und angewandte Mathematik. 683, pp.1-47, 2012-03-10. De Gruyter

バージョン :

権利関係 : ๑ De Gruyter 2013 


\title{
Unified approach to the classification of actions of discrete amenable groups on injective factors
}

\author{
By Toshihiko Masuda at Fukuoka
}

\begin{abstract}
We present a simple unified proof of the classification of discrete amenable group actions on injective factors. Our argument does not depend on the types of factors, and is based on the technique of Evans and Kishimoto.
\end{abstract}

\section{Introduction}

In the theory of operator algebras, the study of automorphism groups and group actions is one of the most important subjects. Especially, since Connes' classification of automorphisms of the injective factor of type $\mathrm{II}_{1}$ up to outer conjugacy in [6] and [3], classification of discrete amenable group actions on injective factors has been developed by many hands. Namely, in the type II case, V. F. R. Jones classified finite group actions in [17], and A. Ocneanu developed the method of Connes and Jones, and classified general discrete amenable group actions in [33]. In the type $\mathrm{III}_{\lambda}(\lambda \neq 1)$ case, C. E. Sutherland and M. Takesaki achieved the classification in $[40,41]$. In the type III $_{1}$ case, classification was obtained by Y. Kawahigashi, Sutherland and Takesaki for finite or abelian group actions [23], and finally by Y. Katayama, Sutherland and Takesaki for arbitrary discrete amenable group actions [20].

Although the classification theorem can be stated in a unified way as in [20] (see Theorem 2.3 for the statement), the proofs presented in the above cited papers heavily depend on the type of factors. Indeed, in the type III cases, they reduce the problem to that of the type II case by means of the structure theorem of type III factors. In this approach, the type $\mathrm{III}_{1}$ case is extremely hard because of the lack of classification of $\mathbb{R}$-actions. This is the reason why type $\mathrm{III}_{\lambda}(\lambda \neq 1)$ and $\mathrm{III}_{1}$ cases are treated separately.

In [30], by developing the Evans-Kishimoto intertwining argument [10], we presented the classification of centrally free actions of discrete amenable groups on injective factors, whose proof is independent from the types of factors. So it is a natural question if one can give a unified approach for classification of general actions by a similar technique. Indeed, for two given actions of a discrete amenable group $G$ with same invariants, if $G$ is a semidirect product $N \rtimes Q$, where $N$ is a centrally trivial part, then we can show the cocycle conjugacy of two actions by using [30].

Supported by the Grand-in-Aid for Young Scientists (B), JSPS. 
In this paper, we extend the method in [30] and present a unified proof of the classification of all actions of discrete amenable groups on injective factors. Our idea for the classification is the following: we first divide a given action to a centrally trivial part and a centrally free part. It is rather easy to classify centrally trivial parts. By applying the intertwining argument, we classify centrally free parts, and combine centrally free parts and centrally trivial parts. A similar idea has already appeared in [19] and [40] for the classification of groupoid actions, where the authors split actions into "group"-parts and "principal-groupoid"-parts, classify and combine them. One of the main difficulties in $[19,40]$ is that cohomological obstruction appears in the combination of two parts. The authors used a special model action to absorb obstructions. A remarkable fact is that no obstruction arises in our argument due to the property of the characteristic invariant and hence we do not need such model actions. Another important point in our approach is that the role of the characteristic invariant in the classification becomes clearer than a model action splitting argument. See $\$ 3.1$ for more detailed explanation on this and the previous paragraph. Our method can be generalized for the classification of $G$-kernels, or outer actions in the sense of [21]. We will discuss this elsewhere.

One difficulty in our argument is that a centrally free part does not give an action in the usual sense because we can not embed a quotient group into a original group as a subgroup, and this makes our argument more difficult. So if we take this fact into account, it is more convenient to handle cocycle crossed actions. Thus we also need to show the second cohomology vanishing. Therefore our argument looks like the mixture of the second cohomology vanishing argument presented in [31] and the intertwining argument. Here we emphasize that our argument is based on the Rohlin type theorem [33] and the characterization of approximately inner automorphisms and centrally trivial automorphisms [5,23].

This paper is organized as follows. In $\S 2$, we recall the definition of invariants of actions and state the main theorem and its corollaries. In $\S 3$, we first classify centrally trivial parts of actions. To treat centrally free parts of actions, we introduce the notion of quasi cocycle crossed actions, and show elementary properties. In $\S 4$, by using ultraproducts, we show that we can approximate a quasi cocycle crossed action by a unitary perturbation of another quasi cocycle crossed action. We also show the approximate one cohomology vanishing, which is the main tool for the intertwining argument. The key ingredient is the Rohlin type theorem. The heart of this paper is $\S 5$, where we classify quasi cocycle crossed actions, and show the main theorem by applying the extended intertwining argument. In $\S 6$, we discuss the construction of model actions. By using Falcone-Takesaki's theory [11], we associate a model action for each fixed faithful normal semifinite weight, and clarify their relation. In §7, we treat group actions on subfactors of type $\mathrm{II}_{1}$ with finite index. By a suitable modification, we can apply the extended intertwining argument in this case. In the appendix, for the readers' convenience, we present proofs of the second cohomology vanishing theorem, the Rohlin theorem and the existence of the extension of automorphisms to a twisted crossed product von Neumann algebra.

Acknowledgement. The author is much indebted and expresses his gratitude to Professor Katayama for improving our argument. He also thanks Professor Takesaki and Professor Ueda for useful comments on this paper. 


\section{Preliminaries and main results}

Our standard reference for the theory of operator algebras is [43]. We assume that every von Neumann algebra has a separable predual except for an ultraproduct algebra, and that every discrete group is at most countable.

2.1. Invariants of actions. First we quickly review the construction of the core covariant system, or the noncommutative flow of weights of a von Neumann algebra, which is necessary to describe invariants of actions.

Let $\mathcal{M}$ be a von Neumann algebra, and $\mathfrak{W}(\mathcal{M})$ be a set of all faithful normal semifinite weights on $\mathcal{M}$. According to $[11,20]$, we associate the core covariant system, or the noncommutative flow of weights $(\tilde{\mathcal{M}}, \mathbb{R}, \theta, \tau)$ with $\mathcal{M}$, where $\tilde{\mathcal{M}}$ is a semifinite von Neumann algebra with a faithful normal semifinite tracial weight $\tau$, and $\theta$ is a one-parameter automorphism group of $\tilde{\mathcal{M}}$ satisfying

$$
\tilde{\mathcal{M}}^{\theta}=\mathcal{M}, \quad \tau \circ \theta_{t}=e^{-t} \tau .
$$

Namely $\tilde{\mathcal{M}}$ is generated by $\mathcal{M}$ and $\left\{\varphi^{i t} \mid \varphi \in \mathfrak{W}(\mathcal{M})\right\}$ with the following relations:

$$
\begin{gathered}
\varphi^{i t} x \varphi^{-i t}=\sigma_{t}^{\varphi}(x), \quad x \in \mathcal{M}, \\
\varphi^{i(t+s)}=\varphi^{i t} \varphi^{i s}, \quad\left(\varphi^{i t}\right)^{*}=\varphi^{-i t}, \quad \varphi^{i t}=[D \varphi: D \psi]_{t} \psi^{i t} .
\end{gathered}
$$

The action $\theta$ is defined as follows:

$$
\theta_{s}\left(x \varphi^{i t}\right)=e^{-i s t} x \varphi^{i t}
$$

Denote $\varphi \circ \alpha^{-1}=\alpha(\varphi)$ for $\alpha \in \operatorname{Aut}(\mathcal{M})$ and $\varphi \in \mathcal{M}_{*}$. By definition, a net of automorphisms $\left(\alpha_{i}\right)_{i} \subset \operatorname{Aut}(\mathcal{M})$ converges to $\alpha$ in the $u$-topology if

$$
\lim _{i}\left\|\alpha_{i}(\varphi)-\alpha(\varphi)\right\|=0
$$

for all $\varphi \in \mathcal{M}_{*}$. The automorphism group $\operatorname{Aut}(\mathcal{M})$ of $\mathcal{M}$ becomes a Polish group with respect to the $u$-topology.

Let $\tilde{\alpha} \in \operatorname{Aut}(\tilde{\mathcal{M}})$ be the canonical extension of $\alpha \in \operatorname{Aut}(\mathcal{M})$; cf. [14]. Namely $\tilde{\alpha}$ is given by

$$
\tilde{\alpha}\left(x \varphi^{i t}\right)=\alpha(x)(\alpha(\varphi))^{i t}=\alpha(x)\left(\varphi \circ \alpha^{-1}\right)^{i t}=\alpha(x)\left[D \varphi \circ \alpha^{-1}: D \varphi\right]_{t} \varphi^{i t} .
$$

Let

$$
\operatorname{Aut}_{\tau, \theta}(\mathcal{M}):=\left\{\sigma \in \operatorname{Aut}(\tilde{\mathcal{M}}) \mid \tau \circ \sigma=\tau, \sigma \circ \theta_{t}=\theta_{t} \circ \sigma, t \in \mathbb{R}\right\} .
$$

Then the canonical extension gives a continuous isomorphism

$$
\alpha \in \operatorname{Aut}(\mathcal{M}) \rightarrow \tilde{\alpha} \in \operatorname{Aut}_{\tau, \theta}(\mathcal{M})
$$

with respect to the $u$-topology.

Denote the flow of weights of $\mathcal{M}$ by $\left(\mathcal{C}, \theta_{t}\right):=\left(Z(\tilde{\mathcal{M}}), \theta_{t}\right)$, and set

$$
\operatorname{Aut}_{\theta}(\mathcal{C})=\left\{\sigma \in \operatorname{Aut}(\mathcal{C}) \mid \sigma \circ \theta_{t}=\theta_{t} \circ \sigma \text { for all } t \in \mathbb{R}\right\}
$$

By the Connes-Takesaki relative commutant theorem [8], we have $\mathcal{M}^{\prime} \cap \tilde{\mathcal{M}}=\mathcal{C}$. If $\mathcal{M}$ is a factor, then $\left(\mathcal{C}, \theta_{t}\right)$ is an ergodic flow. 
The Connes-Takesaki module of $\alpha$ is defined by (see $[8,11,14]$ )

$$
\bmod (\alpha)=\left.\tilde{\alpha}\right|_{e} \in \operatorname{Aut}_{\theta}(e)
$$

Let $\tilde{U}(\mathcal{M})$ be the normalizer unitary group for $\mathcal{M} \subset \tilde{\mathcal{M}}$, which is called the extended unitary group of $\mathcal{M}$. Set

$$
\operatorname{Cnt}_{r}(\mathcal{M})=\{\sigma \in \operatorname{Aut}(\mathcal{M}) \mid \tilde{\sigma} \in \operatorname{Int}(\tilde{\mathcal{M}})\}=\left\{\left.\operatorname{Ad}(u)\right|_{\mathcal{M}} \mid u \in \tilde{U}(\mathcal{M})\right\} .
$$

We have the following identification of the core covariant system with a usual crossed product construction. Take $\psi \in \mathfrak{W}(\mathcal{M})$. Then we can identify $\left(\tilde{\mathcal{M}}, \mathbb{R}, \theta_{t}\right)$ with the crossed product $\left(M \rtimes_{\sigma \psi} \mathbb{R}, \mathbb{R}, \widehat{\sigma}_{t}\right)$ in the canonical way. Let $\lambda^{\psi}(t)$ be the implementing unitary. Via the above identification, $\tilde{\alpha}$ is given by

$$
\left.\tilde{\alpha}\right|_{\mathcal{M}}=\alpha, \quad \tilde{\alpha}\left(\lambda^{\psi}(t)\right)=\left[D \psi \circ \alpha^{-1}: D \psi\right]_{t} \lambda^{\psi}(t) .
$$

See [11] for more on the functorial property of $\left(\tilde{\mathcal{M}}, \mathbb{R}, \theta_{t}, \tau\right)$.

If $\mathcal{M}$ is an injective factor, the Connes-Takesaki module map is surjective. Moreover the following exact sequence splits [42, Corollary 1.3]:

$$
1 \longrightarrow \operatorname{Ker}(\bmod ) \longrightarrow \operatorname{Aut}(\mathcal{M}) \stackrel{\bmod }{\longrightarrow} \operatorname{Aut}_{\theta}(\mathcal{C}) \longrightarrow 1 \text {. }
$$

Now, we will give the definition of cocycle crossed actions and their cocycle conjugacy relation. For groups $G_{i}(1 \leq i \leq n)$ and $H$, let

$$
\begin{aligned}
\operatorname{Map}_{0}\left(G_{1} \times G_{2}\right. & \left.\times \cdots \times G_{n}, H\right) \\
& =\left\{f: G_{1} \times \cdots \times G_{n} \rightarrow H \mid f\left(g_{1}, g_{2}, \ldots, g_{n}\right)=1 \text { if any of } g_{i} \text { is } 1\right\} .
\end{aligned}
$$

When involved groups are Polish, maps are assumed to be Borel.

Definition 2.1. Let $\mathcal{M}$ be a von Neumann algebra, and $G$ a locally compact group.

(1) A cocycle crossed action of $G$ on $\mathcal{M}$ is a pair of maps

$$
(\alpha, a) \in \operatorname{Map}_{0}(G, \operatorname{Aut}(\mathcal{M})) \times \operatorname{Map}_{0}(G \times G, \mathcal{U}(\mathcal{M}))
$$

such that

$$
\alpha_{g} \circ \alpha_{h}=\operatorname{Ad}(a(g, h)) \circ \alpha_{g h}, \quad a(g, h) a(g h, k)=\alpha_{g}(a(h, k)) a(g, h k) .
$$

The latter identity is called the 2-cocycle identity, and we say that $a(\cdot, \cdot)$ is a 2-cocycle for $\alpha$. If $a(\cdot, \cdot) \equiv 1$, then $\left(\alpha_{g}, 1\right)$ is an action of $G$.

(2) Let $\left(\alpha_{g}, a\right)$ be a cocycle crossed action of $G$ on $\mathcal{M}$. For $w \in \operatorname{Map}_{0}(G, \mathcal{U}(\mathcal{M}))$, define $\left({ }_{w} \alpha, w_{w} a\right) \in \operatorname{Map}_{0}(G, \operatorname{Aut}(M)) \times \operatorname{Map}_{0}(G \times G, \mathcal{U}(\mathcal{M}))$ by

$$
{ }_{w} \alpha_{g}=\operatorname{Ad}(w(g)) \circ \alpha_{g}, \quad{ }_{w} a(g, h)=w(g) \alpha_{g}(w(h)) a(g, h) w(g h)^{*} .
$$

Then $\left({ }_{w} \alpha, w a\right)$ is also a cocycle crossed action of $G$, and is called a unitary perturbation of $(\alpha, a)$ by $w(\cdot)$. 
(3) Let $\left(\alpha_{g}, a\right)$ be a cocycle crossed action. If there exists $w \in \operatorname{Map}_{0}(G, \mathcal{U}(\mathcal{M}))$ so that ${ }_{w} a \equiv 1$, then we say that $a(\cdot, \cdot)$ is a coboundary.

(4) Let $(\alpha, 1)$ be a (genuine) action. If a map $w \in \operatorname{Map}_{0}(G, \mathcal{U}(\mathcal{M}))$ satisfies $w(g) \alpha_{g}(w(h))=w(g h)$, then we say that $w$ is a 1-cocycle for $\alpha$. In this case, the perturbation of $(\alpha, 1)$ by $w$ is $(w \alpha, 1)$ and thus it is also a genuine action. Denote by $Z_{\alpha}^{1}(G, \mathcal{U}(\mathcal{M}))$ the set of all 1-cocycles for $\alpha$.

(5) Two cocycle crossed actions $(\alpha, a)$ and $(\beta, b)$ are said to be cocycle conjugate if there exist $w \in \operatorname{Map}_{0}(G, \mathcal{U}(\mathcal{M}))$ and $\theta \in \operatorname{Aut}(\mathcal{M})$ such that

$$
{ }_{w} \alpha_{g}=\theta \circ \beta_{g} \circ \theta^{-1}, \quad{ }_{w} a(g, h)=\theta(b(g, h)), \quad g, h \in G .
$$

If $\theta$ is taken in $\operatorname{Ker}(\mathrm{mod})$, then we say that they are strongly cocycle conjugate.

We will formulate invariants of cocycle crossed actions in the term of $\left(\tilde{\mathcal{M}}, \mathbb{R}, \theta, \tau_{\tilde{\mathcal{M}}}\right)$ as the generalization of invariants of group actions [41]. In the remainder of this paper, we assume that $\mathcal{M}$ is a factor, and $G$ a discrete group.

Let $\left(\alpha_{g}, a\right)$ be a cocycle crossed action of $G$ on $\mathcal{M}$. Then the normal subgroup of $G$ defined by

$$
N=\left\{g \in G \mid \alpha_{g} \in \mathrm{Cnt}_{r}(\mathcal{M})\right\}
$$

is the first invariant.

By restricting $\tilde{\alpha}_{g}$ on $\ell$, we get an action $\bmod \left(\alpha_{g}\right)$ of $G$ on $\ell$, which is the second invariant. Note that $\bmod \left(\alpha_{n}\right)=$ id for $n \in N$. Thus we can regard $\bmod (\alpha)$ as an action of $G / N$ on $\mathcal{C}$. Also note that $\bmod \left(\alpha_{g}\right) \in \operatorname{Aut}_{\theta}(\mathcal{C})$.

The third invariant is a characteristic invariant. Set

$$
d(a)(n, g):=a\left(g, g^{-1} n g\right) a(n, g)^{*}
$$

for a 2-cocycle $a(\cdot, \cdot)$. It is easy to see that

$$
\alpha_{g} \circ \alpha_{g^{-1} n g}=\operatorname{Ad}(d(a)(n, g)) \circ \alpha_{n} \circ \alpha_{g} .
$$

Take $\tilde{u}^{\alpha} \in \operatorname{Map}_{0}(N, \tilde{u}(\mathcal{M}))$ such that $\tilde{\alpha}_{m}=\operatorname{Ad}\left(\tilde{u}^{\alpha}(m)\right), m \in N$. Comparing

$$
\tilde{\alpha}_{g} \circ \tilde{\alpha}_{g^{-1} n g} \circ \tilde{\alpha}_{g}^{-1}=\operatorname{Ad} d(a)(n, g) \circ \tilde{\alpha}_{n} \circ \tilde{\alpha}_{g} \circ \tilde{\alpha}_{g}^{-1}=\operatorname{Ad}\left(d(a)(n, g) \tilde{u}^{\alpha}(n)\right)
$$

with

$$
\tilde{\alpha}_{g} \circ \tilde{\alpha}_{g^{-1} n g} \circ \tilde{\alpha}_{g}^{-1}=\operatorname{Ad}\left(\tilde{\alpha}_{g}\left(\tilde{u}^{\alpha}\left(g^{-1} n g\right)\right)\right),
$$

we get $\lambda \in \operatorname{Map}_{0}(N \times G, U(\mathcal{C}))$ such that

$$
\tilde{\alpha}_{g}\left(\tilde{u}^{\alpha}\left(g^{-1} n g\right)\right)=\lambda(n, g) d(a)(n, g) \tilde{u}^{\alpha}(n) .
$$

In a similar way, from $\tilde{\alpha}_{m} \circ \tilde{\alpha}_{n}=\operatorname{Ad}(a(m, n)) \circ \tilde{\alpha}_{m n}$, we get $\mu \in \operatorname{Map}_{0}(N \times N, \mathcal{U}(\mathcal{C}))$ such that

$$
\tilde{u}^{\alpha}(m) \tilde{u}^{\alpha}(n)=\mu(m, n) a(m, n) \tilde{u}^{\alpha}(m n), \quad m, n \in N .
$$

Since $\theta_{t}$ commutes with $\tilde{\alpha}_{g}$, we get a 1-cocycle $\{c(m, t) \mid t \in \mathbb{R}\} \in Z_{\theta}^{1}(\mathbb{R}, \mathcal{U}(\mathcal{C}))$ by

$$
\theta_{t}\left(\tilde{u}^{\alpha}(m)\right)=c(m, t) \tilde{u}^{\alpha}(m) .
$$


The following relations between $(\lambda, \mu)$ and $c(n, t)$ are easily obtained from $\tilde{\alpha}_{g} \theta_{t}=\theta_{t} \tilde{\alpha}_{g}$ :

$$
\begin{gathered}
\theta_{t}(\lambda(n, g)) c(n, t)=\bmod \left(\alpha_{g}\right)\left(c\left(g^{-1} n g, t\right)\right) \lambda(n, g), \\
c(m, t) c(n, t) \mu(m, n)=c(m n, t) \theta_{t}(\mu(m, n)) .
\end{gathered}
$$

Set $\tilde{G}=G \times \mathbb{R}$, and we extend a cocycle crossed action $(\tilde{\alpha}, a)$ of $G$ to that of $\tilde{G}$ by setting

$$
\tilde{\alpha}_{(g, t)}=\tilde{\alpha}_{g} \theta_{t}, \quad a((g, t),(h, s))=a(g, h) .
$$

Put

$$
\lambda(n,(g, t))=\theta_{t}(\lambda(n, g)) c(n, t), \quad n \in N,(g, t) \in \tilde{G} .
$$

Obviously, $\lambda \in \operatorname{Map}_{0}(N \times \tilde{G}, \mathcal{U}(\mathcal{C}))$. One can verify that $(\lambda, \mu)$ satisfies the following relations, which include the above ones:

$$
\begin{gathered}
\mu(l, m) \mu(l m, n)=\mu(m, n) \mu(l, m n), \quad l, m, n \in N, \\
\lambda(n, g h)=\lambda(n, g) \tilde{\alpha}_{g}\left(\lambda\left(g^{-1} n g, h\right)\right), \quad g, h \in \tilde{G}, n \in N, \\
\lambda(m, g) \lambda(n, g) \lambda(m n, g)^{*}=\tilde{\alpha}_{g}\left(\mu\left(g^{-1} m g, g^{-1} n g\right)\right) \mu(m, n)^{*}, \quad g \in \tilde{G}, m, n \in N, \\
\lambda(n, m)=\mu\left(m, m^{-1} n m\right) \mu(n, m)^{*}, \quad m, n \in N, \\
\lambda(n, g)=\mu(l, m)=1 \text { if any of } g, l, m, n \text { is neutral. }
\end{gathered}
$$

Definition 2.2. (1) We call any $(\lambda, \mu)$ satisfying the above conditions a characteristic cocycle for $\alpha$ and denote the set of all characteristic cocycles by $Z_{\alpha}(\tilde{G}, N, \mathcal{U}(\mathcal{C}))$. Note that $Z_{\alpha}(\tilde{G}, N, \mathcal{U}(\mathcal{C}))$ becomes an abelian group by the natural multiplication.

(2) For $z \in \operatorname{Map}_{0}(N, U(\mathcal{C}))$, define

$$
\partial_{1}(z) \in \operatorname{Map}_{0}(N \times \tilde{G}, \mathcal{U}(\mathcal{C})), \quad \partial_{2}(z) \in \operatorname{Map}_{0}(N \times N, \mathcal{U}(\mathcal{C}))
$$

as

$$
\partial_{1}(z)(n, g)=\tilde{\alpha}_{g}\left(z\left(g^{-1} n g\right)\right) z(n)^{*}, \quad \partial_{2}(z)(m, n)=z(m) z(n) z(m n)^{*} .
$$

Let $B_{\alpha}(\tilde{G}, N, \mathcal{U}(\mathcal{C}))$ be a set of coboundaries defined by

$$
B_{\alpha}(\tilde{G}, N, \mathcal{U}(\mathcal{C}))=\left\{\left(\partial_{1}(z), \partial_{2}(z)\right) \mid z \in \operatorname{Map}_{0}(N, \mathcal{U}(\mathcal{C}))\right\},
$$

which is a normal subgroup of $Z_{\alpha}(\tilde{G}, N, U(\mathcal{C}))$.

(3) Let

$$
\Lambda_{\alpha}(\tilde{G}, N, \mathcal{U}(\mathcal{C}))=Z_{\alpha}(\tilde{G}, N, \mathcal{U}(\mathcal{C})) / B_{\alpha}(\tilde{G}, N, \mathcal{U}(\mathcal{C}))
$$

be a quotient group. The equivalence class $\chi(\alpha)=[\lambda, \nu] \in \Lambda_{\alpha}(\tilde{G}, N, \mathcal{U}(\mathcal{C}))$ is called the characteristic invariant of $\alpha$.

Though $(\lambda, \mu)$ depends on the choice of $\tilde{u}^{\alpha}(n)$, the class $\chi(\alpha)$ does not depend on the choice of $\tilde{u}^{\alpha}(n)$. Thus a true invariant for $\alpha$ is $\chi(\alpha)$ rather than $(\lambda, \mu)$. We denote the triplet $\{N, \bmod (\alpha), \chi(\alpha)\}$ by $\operatorname{Inv}(\alpha)$. 
2.2. Main theorem and its corollaries. We state the main theorem of this paper.

Theorem 2.3. Let $\mathcal{M}$ be a factor, and $G$ a discrete group.

(1) Let $(\alpha, a)$ and $(\beta, b)$ be cocycle crossed actions of $G$ on $\mathcal{M}$. If they are strongly cocycle conjugate, then $\operatorname{Inv}(\alpha)=\operatorname{Inv}(\beta)$.

(2) Assume $\mathcal{M}$ is injective and $G$ is amenable. Let $(\alpha, a)$ and $(\beta, b)$ be cocycle crossed actions of $G$ on $\mathcal{M}$ with $\operatorname{Inv}(\alpha)=\operatorname{Inv}(\beta)$. Then $\alpha$ and $\beta$ are strongly cocycle conjugate. In particular, two genuine actions $\alpha$ and $\beta$ of a discrete amenable group $G$ on an injective factor $\mathcal{M}$ are strongly cocycle conjugate if and only if $\operatorname{Inv}(\alpha)=\operatorname{Inv}(\beta)$.

It is a routine work to show Theorem 2.3 (1), thus we leave the proof to the readers.

The main purpose of this paper is to present a unified proof of Theorem 2.3 (2), which does not depend on the types of factors.

Here we explain the reason why we treat cocycle crossed actions in this article, although the main interest is the classification of (genuine) actions. To obtain a new (genuine) action from a given action $\alpha_{g}$ by perturbing with $w(\cdot)$, we need the 1-cocycle identity $w(g) \alpha_{g}(w(h))=w(g h)$, which can be regard as a "global condition", and it is not so easy to take a 1-cocycle for general actions. On the other hand, a simple unitary perturbation does not require a "global condition" like the 1-cocycle identity, and we can perturb (cocycle crossed) actions "locally". As will be explained in $§ 3.1$, we split actions to "centrally trivial parts" and "centrally free parts" and treat them separately in our argument. Thus local perturbation is more convenient for our purpose.

As in [30], our strategy for the proof is the Evans-Kishimoto type intertwining argument [10]. The proof will be presented in the subsequent sections. Thus, in this subsection, we only state corollaries of the main theorem.

For $\sigma \in \operatorname{Aut}_{\theta}(\mathcal{C})$, we denote

$$
\sigma(\operatorname{Inv}(\alpha))=\left\{N, \sigma \circ \bmod \left(\alpha_{g}\right) \circ \sigma^{-1},[\sigma(\lambda), \sigma(\mu)]\right\} .
$$

Corollary 2.4. Let $\mathcal{M}$ and $G$ be as in Theorem 2.3. Let $\alpha$ and $\beta$ be actions of $G$. Then $\alpha$ and $\beta$ are cocycle conjugate if and only if there exists $\sigma \in \operatorname{Aut}_{\theta}(\mathcal{C})$ such that the equality $\operatorname{Inv}(\alpha)=\sigma(\operatorname{Inv}(\beta))$ holds.

Proof. This follows from Theorem 2.3, $\operatorname{Inv}\left(\gamma \circ \alpha \circ \gamma^{-1}\right)=\bmod (\gamma)(\operatorname{Inv}(\alpha))$, and the surjectivity of the module map $\gamma \in \operatorname{Aut}(\mathcal{M}) \rightarrow \bmod (\gamma) \in \operatorname{Aut}_{\theta}(\mathcal{C})$ (see [42, Theorem 1.1]).

Since there exists a genuine action $\beta$ with $\operatorname{Inv}(\alpha)=\operatorname{Inv}(\beta)$ by [17, Proposition 1.5.8], [41, Theorem 5.14], [23, Proposition 22], the following corollary immediately follows from Theorem 2.3. (Also see $\S 6$ for the construction of model actions.)

Corollary 2.5. The second cohomology of a cocycle crossed action of a discrete amenable group on the injective factor of type $\mathrm{II}_{1}$ vanishes. Namely, 2-cocycles of any cocycle actions are coboundaries.

Remark. So far, it is known that the second cohomology vanishing theorem holds in the following cases (see $[17,33,39])$ : 
(1) arbitrary cocycle crossed actions of free groups on arbitrary von Neumann algebras,

(2) arbitrary cocycle crossed actions of arbitrary locally compact groups on properly infinite von Neumann algebras,

(3) arbitrary cocycle crossed actions of finite groups on type $\mathrm{II}_{1}$ von Neumann algebras,

(4) centrally free cocycle crossed actions of discrete amenable groups on McDuff factors.

In particular, for infinite discrete amenable groups and the injective factor of type $\mathrm{II}_{1}$, the second cohomology vanishing theorem has been known for only free cocycle crossed actions. Hence the above corollary removes the assumption of freeness in this case. If we do not have the condition of amenability of discrete groups in (4), the second cohomology does not necessary vanish [35].

2.3. Cohomological approach to definition of invariants. In this subsection, we give a cohomological explanation of the definition of $\chi(\alpha)$ for a cocycle crossed action $(\alpha, a)$. We consider an extended cocycle crossed action $(\tilde{\alpha}, a)$ of $\tilde{G}$ on $\tilde{\mathcal{M}}$ defined as above.

We define a multiplication of $\tilde{U}(\mathcal{M}) \times \tilde{G}$ by

$$
(u, g)(w, h)=\left(u \tilde{\alpha}_{g}(w) a(g, h), g h\right)
$$

and denote this group by $\tilde{U}(\mathcal{M}) \rtimes_{a} \tilde{G}$. Indeed, the 2-cocycle identity of $a(\cdot, \cdot)$ assures that this multiplication is associative. If an appearing 2-cocycle is clear, then we simply write $\tilde{U}(\mathcal{M}) \rtimes \tilde{G}$. We can easily verify

$$
(1, g)\left(1, g^{-1} n g\right)=(d(a)(n, g), n)(1, g) .
$$

Define a subgroup $\bar{N} \subset \mathcal{U}(\tilde{\mathcal{M}}) \rtimes \tilde{G}$ by

$$
\bar{N}:=\left\{\left(u^{*}, n\right) \mid n \in N \text { and } \operatorname{Ad}(u)=\tilde{\alpha}_{n}\right\} .
$$

Then $\bar{N}$ is a normal subgroup of $\tilde{U}(\mathcal{M}) \rtimes \tilde{G}$. Indeed, since we have

$$
\left(u^{*}, m\right)\left(w^{*}, n\right)=\left(u^{*} \tilde{\alpha}_{n}\left(w^{*}\right) a(m, n), m n\right)=\left(w^{*} u^{*} a(m, n), m n\right)
$$

and

$$
\operatorname{Ad}\left(a(m, n)^{*} u w\right)=\operatorname{Ad}\left(a(m, n)^{*}\right) \circ \tilde{\alpha}_{m} \circ \tilde{\alpha}_{n}=\tilde{\alpha}_{m n},
$$

$\bar{N}$ is a subgroup. To see that $\bar{N}$ is normal, we only have to verify

$$
(w, e)\left(u^{*}, n\right)(w, e)^{-1},(1, g)\left(u^{*}, g^{-1} n g\right)(1, g)^{-1} \in \bar{N} .
$$

In fact, $\tilde{U}(\mathcal{M})$ are in the commutant of $\bar{N}$ as shown below.

$$
(w, e)\left(u^{*}, n\right)(w, e)^{-1}=\left(w u^{*}, n\right)\left(w^{*}, e\right)=\left(w u^{*} \tilde{\alpha}_{n}\left(w^{*}\right), n\right)=\left(u^{*}, n\right) .
$$

Next we have the following:

$$
\begin{aligned}
(1, g)\left(u^{*}, g^{-1} n g\right)(1, g)^{-1} & =\left(\tilde{\alpha}_{g}\left(u^{*}\right), e\right)(1, g)\left(1, g^{-1} n g\right)(1, g)^{-1} \\
& =\left(\tilde{\alpha}_{g}\left(u^{*}\right) d(a)(n, g), n\right),
\end{aligned}
$$


and

$$
\begin{aligned}
\operatorname{Ad}\left(d(a)(n, g)^{*} \tilde{\alpha}_{g}(u)\right) & =\operatorname{Ad}\left(d(a)(n, g)^{*}\right) \circ \tilde{\alpha}_{g} \circ \operatorname{Ad}(u) \circ \tilde{\alpha}_{g}^{-1} \\
& =\operatorname{Ad}\left(d(a)(n, g)^{*}\right) \circ \tilde{\alpha}_{g} \circ \tilde{\alpha}_{g^{-1} n g} \circ \tilde{\alpha}_{g}^{-1}=\tilde{\alpha}_{n}
\end{aligned}
$$

Hence $(1, g)\left(u^{*}, g^{-1} n g\right)(1, g)^{-1} \in \bar{N}$. The above computation shows that

$$
\left(u^{*}, n\right) \rightarrow(1, g)\left(u^{*}, n\right)(1, g)^{-1}
$$

defines an action of $\tilde{G}$ on $\bar{N}$ due to $[\tilde{U}(\mathcal{M}), \bar{N}]=\{(1, e)\}$, although $\{(1, g) \mid g \in G\}$ is not a subgroup of $\tilde{U}(\mathcal{M}) \rtimes_{a} \tilde{G}$.

We have the following $\tilde{G}$-equivariant exact sequence:

$$
0 \longrightarrow U(\bigodot) \stackrel{\iota}{\longrightarrow} \bar{N} \stackrel{\pi}{\longrightarrow} N \longrightarrow 0
$$

where $\iota(z)=\left(z^{*}, e\right) \in \bar{N}$, and $\pi\left(u^{*}, n\right)=n$. The characteristic invariant associated with this exact sequence is nothing but $\chi(\alpha)$. See $[17,41]$ for a cohomological property of $\chi(\alpha)$.

Remark. Let $\tilde{\mathcal{M}} \rtimes_{\tilde{\alpha}, a} \tilde{G}$ be a twisted crossed product, and $\lambda(g)$ the implementing unitary. The group $\tilde{U}(\mathcal{M}) \rtimes \tilde{G}$ is identified with

$$
\{u \lambda(g) \mid u \in \tilde{U}(\mathcal{M}), g \in \tilde{G}\},
$$

and we have

$$
\bar{N}=\left\{u^{*} \lambda(h) \mid h \in N, \operatorname{Ad}(u)=\tilde{\alpha}_{h}\right\}
$$

via this identification.

The above argument can be generalized by using the characteristic square of $\mathcal{M}$ (see [20]). The characteristic square for $\mathcal{M}$ is the following nine-term exact square:

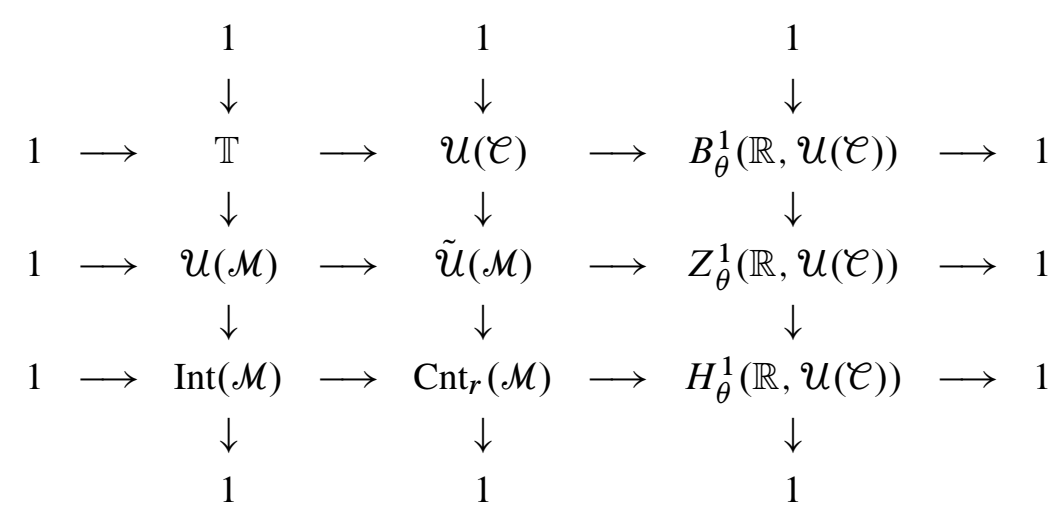

From the characteristic square, we obtain the intrinsic invariant $\Theta(\mathcal{M})$. Assume $\alpha$ is an action, i.e., $a(g, h)=1$, for simplicity. Then $\chi(\alpha)$ is obtained by the pull-back $\chi(\alpha)=\alpha_{*}(\Theta(\mathcal{M}))$. See [20, Section 2] for details. 


\section{Quasi cocycle crossed actions}

3.1. Outline of the proof of the main theorem. In the rest of this paper, $\mathcal{M}$ and $G$ always denote an injective factor and a discrete amenable group, respectively.

Let $(\alpha, a)$ and $(\beta, b)$ be as in Theorem 2.3. In this case, we can choose maps $\tilde{u}^{\alpha}, \tilde{u}^{\beta} \in \operatorname{Map}_{0}(N, \mathcal{U}(\tilde{\mathcal{M}}))$ such that

$$
\begin{aligned}
\tilde{\alpha}_{g}\left(\tilde{u}^{\alpha}\left(g^{-1} n g\right)\right) & =\lambda(n, g) d(a)(n, g) \tilde{u}^{\alpha}(n), \\
\tilde{u}^{\alpha}(m) \tilde{u}^{\alpha}(n) & =\mu(m, n) a(m, n) \tilde{u}^{\alpha}(m n), \\
\theta_{t}\left(\tilde{u}^{\alpha}(m)\right) & =c(m, t) \tilde{u}^{\alpha}(m), \\
\tilde{\beta}_{g}\left(\tilde{u}^{\beta}\left(g^{-1} n g\right)\right) & =\lambda(n, g) d(b)(n, g) \tilde{u}^{\beta}(n), \\
\tilde{u}^{\beta}(m) \tilde{u}^{\beta}(n) & =\mu(m, n) b(m, n) \tilde{u}^{\beta}(m n), \\
\theta_{t}\left(\tilde{u}^{\beta}(m)\right) & =c(m, t) \tilde{u}^{\beta}(m) .
\end{aligned}
$$

To explain our idea of the proof of Theorem 2.3, we consider the following special case. Set $Q=G / N$. Assume that $a(g, h)=b(g, h)=1$, and $G$ is of the form $G=N \rtimes Q$, i.e., $Q$ is a subgroup of $G$. Then $\left.\alpha\right|_{Q}$ and $\left.\beta\right|_{Q}$ are actions of $Q$ on $\mathcal{M}$ with $\alpha_{p} \circ \beta_{p}^{-1} \in \operatorname{Ker}(\bmod )$, and $\alpha_{q}, \beta_{q} \notin \mathrm{Cnt}_{r}(\mathcal{M}), 1 \neq q \in Q$. By [30], $\left.\alpha\right|_{Q}$ and $\left.\beta\right|_{Q}$ are strongly cocycle conjugate, i.e., we have

$$
\operatorname{Ad}(v(p)) \circ \alpha_{p}=\sigma \circ \beta_{p} \circ \sigma^{-1}
$$

for some $\alpha$-cocycle $\{v(p)\}_{p \in Q}$ and $\sigma \in \operatorname{Ker}(\bmod )$. Put $v(n)=\sigma\left(\tilde{u}^{\beta}(n)\right) \tilde{u}^{\alpha}(n)^{*}$ for $n \in N$. By the choice of $\tilde{u}^{\alpha}(n)$ and $\tilde{u}^{\beta}(n)$, we can verify that $v(n) \in \tilde{\mathcal{M}}^{\theta}=\mathcal{M}$, and $\{v(n)\}_{n \in N}$ is a 1-cocycle for $\left.\alpha\right|_{N}$ with

$$
\operatorname{Ad}(v(n)) \circ \alpha_{n}=\sigma \circ \beta_{n} \circ \sigma^{-1} .
$$

(See Lemma 3.1 below.) We then define $v(n p)=v(n) \alpha_{n}(v(p))$ for $n \in N, p \in Q$. We can verify $v(p) \alpha_{p}(v(n))=v(p n)$ for $p \in Q$ and $n \in N$ as follows:

$$
\begin{aligned}
v(p) \alpha_{p}(v(n)) & =v(p) \tilde{\alpha}_{p}\left(\sigma\left(\tilde{u}^{\beta}(n)\right) \tilde{u}^{\alpha}(n)^{*}\right) \\
& =\sigma \circ \tilde{\beta}_{p}\left(\tilde{u}^{\beta}(n)\right) v(p) \tilde{\alpha}_{p}\left(\tilde{u}^{\alpha}(n)^{*}\right) \\
& =\sigma\left(\lambda\left(p n p^{-1}, p\right) \tilde{u}^{\beta}\left(p n p^{-1}\right)\right) v(p) \lambda\left(p n p^{-1}, p\right)^{*} \tilde{u}^{\alpha}\left(p n p^{-1}\right)^{*} \\
& =\sigma\left(\tilde{u}^{\beta}\left(p n p^{-1}\right)\right) v(p) \tilde{u}^{\alpha}\left(p n p^{-1}\right)^{*} \\
& =\sigma\left(\tilde{u}^{\beta}\left(p n p^{-1}\right)\right) \tilde{u}^{\alpha}\left(p n p^{-1}\right)^{*} \alpha_{p n p^{-1}}(v(p)) \\
& =v\left(p n p^{-1}\right) \alpha_{p n p^{-1}}(v(p))=v(p n) .
\end{aligned}
$$

It follows that $\{v(g)\}_{g \in G}$ becomes a 1-cocycle for $\alpha$ and we have

$$
\operatorname{Ad}(v(g)) \circ \alpha_{g}=\sigma \circ \beta_{g} \circ \sigma^{-1}, \quad g \in G .
$$

Thus $\alpha$ and $\beta$ are strongly cocycle conjugate.

If we compare our argument with the model action splitting argument, the role of characteristic invariants in classification becomes clearer. Namely, the information of two invariants $\mu(\cdot, \cdot)$ and $c(\cdot, \cdot)$ assures the cocycle conjugacy of $N$-parts of actions. The information on Connes-Takesaki modules assures the cocycle conjugacy of $Q$-parts of actions. Thus $N$-parts 
and $Q$-parts are separately cocycle conjugate. To combine these parts, we use the information of $\lambda(\cdot, \cdot)$. If we do not have the information of $\lambda(\cdot, \cdot)$, then an obstruction may arise as a difference of $v(p) \alpha_{p}(v(q))$ and $v\left(p n p^{-1}\right) \alpha_{p n p^{-1}}(v(p))$. In fact, such obstruction arises in the classification of groupoid actions in [19], and one must construct the model action which absorb obstructions to complete the classification.

We would like to extend the above argument to general case. The main difficulty is that $G$ is not a semidirect product of $N$ by $Q$, that is, we can not embed $Q$ into $G$ as a subgroup. So $\alpha$ does not give an action of $Q$. To treat such case, we introduce the notion of quasi cocycle crossed cocycle actions of $Q$. We first classify two quasi cocycle actions of $Q$ by the intertwining argument, and combine $N$-parts and $Q$-parts as above. In what follows, we mainly use letters $g, h, k$ for elements of $G$, letters $m, n$ for elements of $N$, and letters $p, q, r$ for elements of $Q$.

3.2. Quasi cocycle crossed actions. We begin with the following lemma, which says that $\alpha$ and $\beta$ are cocycle conjugate as actions of $N$.

Lemma 3.1. Define $u(m)=\tilde{u}^{\alpha}(m) \tilde{u}^{\beta}(m)^{*}$. Then $u(m) \in \mathcal{M}$ and we have

$$
\alpha_{m}=\operatorname{Ad}(u(m)) \circ \beta_{m}, \quad a(m, n)=u(m) \beta_{m}(u(n)) b(m, n) u(m n)^{*} .
$$

Proof. By the choice of $\tilde{u}^{\alpha}(m)$ and $\tilde{u}^{\beta}(m)$, we have $\theta_{t}(u(m))=u(m) \in \tilde{\mathcal{M}}^{\theta}=\mathcal{M}$. It is clear that $\alpha_{m}=\operatorname{Ad}(u(m)) \circ \beta_{m}$, since $\tilde{\alpha}_{m}=\operatorname{Ad}(u(m)) \circ \tilde{\beta}_{m}$. The last equation can be verified as follows:

$$
\begin{aligned}
u(m) & \beta_{m}(u(n)) b(m, n) u(m n)^{*} \\
& =\tilde{u}^{\alpha}(m) \tilde{u}^{\beta}(m)^{*} \tilde{\beta}_{m}\left(\tilde{u}^{\alpha}(n) \tilde{u}^{\beta}(n)^{*}\right) b(m, n) \tilde{u}^{\beta}(m n) \tilde{u}^{\alpha}(m n)^{*} \\
& =\tilde{u}^{\alpha}(m) \tilde{u}^{\alpha}(n) \tilde{u}^{\beta}(n)^{*} \tilde{u}^{\beta}(m)^{*} b(m, n) \tilde{u}^{\beta}(m n) \tilde{u}^{\alpha}(m n)^{*} \\
& =\mu(m, n)^{*} \tilde{u}^{\alpha}(m) \tilde{u}^{\alpha}(n) \tilde{u}^{\alpha}(m n)^{*}=a(m, n) .
\end{aligned}
$$

Remark. Consider a unitary perturbation of $(\alpha, a)$ by $w \in \operatorname{Map}_{0}(G, \mathcal{U}(\mathcal{M}))$. If we choose $\tilde{u}^{w \alpha}(n)$ as $w(n) \tilde{u}^{\alpha}(n)$, then $\left(\alpha, a, \tilde{u}^{\alpha}\right)$ and $\left({ }_{w} \alpha,{ }_{w} a, \tilde{u}^{w}{ }^{\alpha}\right)$ produce the same characteristic cocycle.

By Lemma 3.1, we can assume $\left(\alpha_{n}, a(m, n)\right)=\left(\sigma_{n}, v(m, n)\right)$ for some fixed cocycle crossed action $\sigma$ of $N$ by a suitable unitary perturbation. Moreover the existence of model actions allows us to further assume $a(m, n)=v(m, n)=1$ for all $m, n \in N$. Although our argument is valid without assuming that $a(m, n)=1$ for $m, n \in N$, we assume this for simplicity. In what follows, we fix $\tilde{u} \in \operatorname{Map}_{0}(N, \tilde{u}(\mathcal{M}))$ with $\tilde{\sigma}_{n}=\operatorname{Ad}(\tilde{u}(n))$.

By the above remark, we have

$$
\begin{gathered}
\tilde{u}(m) \tilde{u}(n)=\mu(m, n) \tilde{u}(m n), \quad \tilde{\alpha}_{g}\left(\tilde{u}\left(g^{-1} n g\right)\right)=\lambda(n, g) d(a)(n, g) \tilde{u}(n), \\
\theta_{t}(\tilde{u}(m))=c(m, t) \tilde{u}(m), \quad \tilde{\beta}_{g}\left(\tilde{u}\left(g^{-1} n g\right)\right)=\lambda(n, g) d(b)(n, g) \tilde{u}(n), \\
\theta_{t}(\tilde{u}(m))=c(m, t) \tilde{u}(m) .
\end{gathered}
$$

We have already classified $N$-parts of actions in Lemma 3.1, and to prove our main theorem, we must classify $Q=G / N$-parts of actions. To treat $Q$-parts, we will introduce the notion of quasi cocycle actions of $Q$. 
Fix a section $(p \in Q \rightarrow \tilde{p} \in G) \in \operatorname{Map}_{0}(Q, G)$, and set

$$
m(p, q)=\widetilde{p q}^{-1} \tilde{p} \tilde{q} \in N .
$$

So $\tilde{p} \tilde{q}=\widetilde{p q} \cdot m(p, q)$ holds. From the associativity condition $(\tilde{p} \tilde{q}) \tilde{r}=\tilde{p}(\tilde{q} \tilde{r})$, we obtain

$$
m(p q, r) \tilde{r}^{-1} m(p, q) \tilde{r}=m(p, q r) m(q, r) .
$$

We denote this element by $m(p, q, r)$. Set $c^{a}(p, q)=a(\tilde{p}, \tilde{q}) a(\widetilde{p q}, m(p, q))^{*}$. We can see that

$$
\begin{aligned}
\alpha_{\tilde{p}} \circ \alpha_{\tilde{q}} & =\operatorname{Ad}(a(\tilde{p}, \tilde{q})) \circ \alpha_{\tilde{p} \tilde{q}} \\
& =\operatorname{Ad}(a(\tilde{p}, \tilde{q})) \circ \alpha_{\tilde{p q} \cdot m(p, q)}=\operatorname{Ad}\left(c^{a}(p, q)\right) \circ \alpha_{\widetilde{p q}} \circ \sigma_{m(p, q)} .
\end{aligned}
$$

Definition 3.2. We call $\left(\alpha_{\tilde{p}}, c^{a}\right)$ a quasi cocycle crossed action of $Q$.

The unitary $c^{a}(p, q)$ behaves like a 2-cocycle as follows.

Lemma 3.3. For $p, q, r \in Q$, we have

$$
c^{a}(p, q) \alpha_{\widetilde{p q}}\left(d(a)(m(p, q), \tilde{r})^{*}\right) c^{a}(p q, r)=\alpha_{\tilde{p}}\left(c^{a}(q, r)\right) c^{a}(p, q r) .
$$

Proof. In $\tilde{U}(\mathcal{M}) \rtimes_{a} \tilde{G}$, we compute $((1, \tilde{p})(1, \tilde{q}))(1, \tilde{r})=(1, \tilde{p})((1, \tilde{q})(1, \tilde{r}))$. First note the following relations:

$$
\begin{gathered}
(1, \tilde{p})(1, \tilde{q})=(a(\tilde{p}, \tilde{q}), \tilde{p} \tilde{q})=\left(c^{a}(p, q), \widetilde{p q}\right)(1, m(p, q)), \\
(1, n)(1, g)=\left(d(a)(n, g)^{*}, e\right)(1, g)\left(1, g^{-1} n g\right) .
\end{gathered}
$$

On one hand, we have

$$
\begin{aligned}
((1, \tilde{p}) & (1, \tilde{q}))(1, \tilde{r}) \\
& =\left(c^{a}(p, q), \widetilde{p q}\right)(1, m(p, q))(1, \tilde{r}) \\
& =\left(c^{a}(p, q), \widetilde{p q}\right)\left(d(a)(m(p, q), \tilde{r})^{*}, e\right)(1, \tilde{r})\left(1, \tilde{r}^{-1} m(p, q) \tilde{r}\right) \\
& =\left(c^{a}(p, q), e\right)\left(\alpha_{\widetilde{p q}}\left(d(a)(m(p, q), \tilde{r})^{*}\right), e\right)(1, \widetilde{p q})(1, \tilde{r})\left(1, \tilde{r}^{-1} m(p, q) \tilde{r}\right) \\
& =\left(c^{a}(p, q) \alpha_{\widetilde{p q}}\left(d(a)(m(p, q), \tilde{r})^{*}\right) c^{a}(p q, r), \widetilde{p q r}\right)(1, m(p q, r))\left(1, \tilde{r}^{-1} m(p, q) \tilde{r}\right) \\
& =\left(c^{a}(p, q) \alpha_{\widetilde{p q}}\left(d(a)(m(p, q), \tilde{r})^{*}\right) c^{a}(p q, r), \widetilde{p q r}\right)(1, m(p, q, r)) .
\end{aligned}
$$

On the other hand, we have

$$
\begin{aligned}
(1, \tilde{p})((1, \tilde{q})(1, \tilde{r})) & =(1, \tilde{p})\left(c^{a}(q, r), \widetilde{q r}\right)(1, m(q, r)) \\
& =\left(\alpha_{\tilde{p}}\left(c^{a}(q, r)\right), e\right)(1, \tilde{p})(1, \widetilde{q r})(1, m(q, r)) \\
& =\left(\alpha_{\tilde{p}}\left(c^{a}(q, r)\right) c^{a}(p, q r), e\right)(1, \widetilde{p q r})(1, m(p, q r))(1, m(q, r)) \\
& =\left(\alpha_{\tilde{p}}\left(c^{a}(q, r)\right) c^{a}(p, q r), \widetilde{p q r}\right)(1, m(p, q, r)) .
\end{aligned}
$$

Note that $a(m, n)=1$ for $m, n \in N$. Thus we get the conclusion. 
Remark. If we do not assume $a(m, n)=1$, then we get

$$
\begin{gathered}
c^{a}(p, q) \alpha_{\widetilde{p q}}\left(d(a)(m(p, q), \tilde{r})^{*}\right) c^{a}(p q, r) \alpha_{\widetilde{p q r}}\left(a\left(m(p q, r), \tilde{r}^{-1} m(p, q) \tilde{r}\right)\right) \\
=\alpha_{\tilde{p}}\left(c^{a}(q, r)\right) c^{a}(p, q r) \alpha_{\widetilde{p q r}}(a(m(p, q r), m(q, r))) .
\end{gathered}
$$

Lemma 3.4. Let $(\alpha, a)$ be a cocycle crossed action of $G$, and $(\gamma, v)$ a unitary perturbation of $\alpha$ by $w \in \operatorname{Map}_{0}(G, \mathcal{U}(\mathcal{M}))$ with $w(n)=1, n \in N$. Then a quasi cocycle crossed action associated with $\gamma$ is given by

$$
\left(\gamma_{\tilde{p}}, c^{v}(p, q)\right)=\left(\operatorname{Ad}(w(\tilde{p})) \circ \alpha_{\tilde{p}}, w(\tilde{p}) \alpha_{\tilde{p}}(w(\tilde{q})) c^{a}(p, q) w(\widetilde{p q})^{*}\right) .
$$

Proof. It is an easy computation as follows:

$$
\begin{aligned}
c^{v}(p, q) & =v(\tilde{p}, \tilde{q}) v(\widetilde{p q}, m(p, q))^{*} \\
& =w(\tilde{p}) \alpha_{\tilde{p}}(w(\tilde{q})) a(\tilde{p}, \tilde{q}) w(\tilde{p} \tilde{q})^{*} w(\tilde{p} \tilde{q}) a(\widetilde{p q}, m(p, q))^{*} \alpha_{\widetilde{p q}}(w(m(p, q))) w(\widetilde{p q})^{*} \\
& =w(\tilde{p}) \alpha_{\tilde{p}}(w(\tilde{q})) c^{a}(p, q) w(\widetilde{p q})^{*} .
\end{aligned}
$$

\section{Asymptotic cocycles and coboundaries}

4.1. Preliminaries on ultraproduct von Neumann algebras. In the rest of the paper, we use the following notation. For $\alpha \in \operatorname{Aut}(\mathcal{M}), a \in \mathcal{M}$ and $\psi \in \mathcal{M}_{*}$, the functionals $\alpha(\psi)$, $a \cdot \psi, \psi \cdot a$ and $[a, \psi]$ are defined as follows:

$$
\alpha(\psi)=\psi \circ \alpha^{-1}, \quad a \cdot \psi(x)=\psi(x a), \quad \psi \cdot a(x)=\psi(a x), \quad[a, \psi]=a \cdot \psi-\psi \cdot a .
$$

Recall that $\left\{\alpha_{\nu}\right\}_{v} \subset \operatorname{Aut}(\mathcal{M})$ converges to $\alpha$ in the $u$-topology if

$$
\lim _{\nu}\left\|\alpha_{\nu}(\psi)-\alpha(\psi)\right\|=0
$$

for all $\psi \in \mathcal{M}_{*}$. The closure of $\operatorname{Int}(\mathcal{M}) \subset \operatorname{Aut}(\mathcal{M})$ with respect to the $u$-topology is the set of approximately inner automorphisms and denoted by $\overline{\operatorname{Int}}(\mathcal{M})$.

It is easy to see that a norm bounded sequence $\left\{a_{\nu}\right\}_{\nu} \subset \mathcal{M}$ converges to $a$ in the $\sigma$-strong* topology if and only if

$$
\lim _{v}\left\|\left(a_{v}-a\right) \cdot \psi\right\|=\lim _{v}\left\|\psi \cdot\left(a_{v}-a\right)\right\|=0
$$

for all $\psi \in \mathcal{M}_{*}$, and equivalently

$$
\lim _{v}\left\|\left(a_{v}-a\right) \cdot \varphi\right\|=\lim _{v}\left\|\varphi \cdot\left(a_{v}-a\right)\right\|=0
$$

for some fixed faithful normal state $\varphi$. The advantage of using these norms instead of the usual norms $\left.\|a\|_{\varphi}^{\#}=\varphi\left(\left(x x^{*}+x^{*} x\right) / 2\right)\right)^{\frac{1}{2}}$ defining the $\sigma$-strong* topology is the unitary invariance of $\|\psi\|$, i.e., $\|u \cdot \psi \cdot v\|=\|\psi\|$ for $\psi \in \mathcal{M}_{*}, u, v \in U(\mathcal{M})$.

We recall the definition of an ultraproduct algebra and its property. See $[2,32,33]$ for details. Fix a free ultrafilter $\omega$ over $\mathbb{N}$. Define three $C^{*}$-subalgebras of $\ell^{\infty}(\mathbb{N}, \mathcal{M})$ as follows:

$$
\begin{aligned}
I_{\omega} & :=\left\{\left(x_{\nu}\right) \in \ell^{\infty}(\mathbb{N}, \mathcal{M}) \mid \lim _{\nu \rightarrow \omega} x_{\nu}=0 \text { in the } \sigma \text {-strong* topology }\right\}, \\
C_{\omega} & :=\left\{\left(x_{\nu}\right) \in \ell^{\infty}(\mathbb{N}, \mathcal{M}) \mid \lim _{\nu \rightarrow \omega}\left\|\left[x_{\nu}, \psi\right]\right\|=0 \text { for all } \psi \in \mathcal{M}_{*}\right\},
\end{aligned}
$$

$\mathcal{N}\left(I_{\omega}\right)$ : the normalizer of $I_{\omega}$. 
Then we have $I_{\omega} \subset C_{\omega} \subset \mathcal{N}\left(I_{\omega}\right)$, and $\mathcal{M} \subset \mathcal{N}\left(I_{\omega}\right)$. The quotient $C^{*}$-algebras

$$
\mathcal{M}_{\omega}=C_{\omega} / I_{\omega}, \quad \mathcal{M}^{\omega}=\mathcal{N}\left(I_{\omega}\right) / I_{\omega}
$$

are in fact both von Neumann algebras. They are called central sequence algebra and ultraproduct algebra, respectively. By the canonical embedding $\mathcal{M} \hookrightarrow \mathcal{M}^{\omega}$, we can regard $\mathcal{M}$ as a von Neumann subalgebra of $\mathcal{M}^{\omega}$. If $\mathcal{M}$ is a factor of type $\mathrm{II}_{1}$, then $\mathcal{M}_{\omega}=\mathcal{M}^{\prime} \cap \mathcal{M}^{\omega}$ holds, but in general we only have $\mathcal{M}_{\omega} \subset \mathcal{M}^{\prime} \cap \mathcal{M}^{\omega}$.

If $\mathcal{M}$ is $\mathrm{McDuff}$ (or strongly stable), i.e., $\mathcal{M} \otimes \mathcal{R}_{0} \cong \mathcal{M}$ where $\mathcal{R}_{0}$ is the injective factor of type $\mathrm{II}_{1}$, then $\mathcal{M}_{\omega}$ is a type $\mathrm{II}_{1}$ von Neumann algebra, and

$$
\tau_{\omega}(X)=\lim _{\nu \rightarrow \omega} x_{v}, \quad X=\left(x_{v}\right) \in \mathcal{M}_{\omega},
$$

defines a tracial state, where the limit is taken in the $\sigma$-weak topology. In particular, $\mathcal{M}_{\omega}$ is of type $\mathrm{II}_{1}$ for an injective factor $\mathcal{M}$, thanks to the Connes-Krieger-Haagerup classification of injective factors $[4,7,13,25]$.

Let $\tau^{\omega}: \mathcal{M}^{\omega} \rightarrow \mathcal{M}$ be a map given by

$$
\tau^{\omega}(X)=\lim _{v \rightarrow \omega} x_{v}, \quad X=\left(x_{v}\right) \in \mathcal{M}^{\omega},
$$

where the limit is taken in the $\sigma$-weak topology. We extend $\varphi \in \mathcal{M}_{*}$ to $\left(\mathcal{M}^{\omega}\right)_{*}$ by

$$
\varphi(X)=\varphi \circ \tau^{\omega}(X)=\lim _{\nu \rightarrow \omega} \varphi\left(x_{v}\right), \quad X=\left(x_{v}\right) \in \mathcal{M}^{\omega} .
$$

We have $\left.\varphi \circ \tau^{\omega}\right|_{\mathcal{M}_{\omega}}=\left.\tau^{\omega}\right|_{\mathcal{M}_{\omega}}=\tau_{\omega}$. We denote the $L^{1}$-norm $\tau_{\omega}(|X|)$ on $\mathcal{M}_{\omega}$ by $\|X\|_{1}$.

We can lift $\alpha \in \operatorname{Aut}(\mathcal{M})$ to $\operatorname{Aut}\left(\mathcal{M}^{\omega}\right)$ in the canonical way. Moreover, assume that $\left\{\alpha_{\nu}\right\}_{\nu=1}^{\infty}$ converges to $\alpha$ in the $u$-topology. Then we can define an automorphism $\gamma \in \operatorname{Aut}\left(\mathcal{M}^{\omega}\right)$ by $\gamma\left(\left(x_{v}\right)\right)=\left(\alpha_{v}\left(x_{v}\right)\right)$. Such $\gamma$ is said to be semiliftable, and we say $\left(\alpha_{v}\right)$ represents $\gamma$. Note that $\gamma(x)=\alpha(x)$ holds for $x \in \mathcal{M}$. It is easy to see that a semiliftable automorphism preserves $\mathcal{M}_{\omega}$. A typical example of semiliftable automorphisms comes from approximately inner ones. Assume

$$
\lim _{\nu \rightarrow \infty} \operatorname{Ad}\left(u_{v}\right)=\alpha, \quad u_{v} \in \mathcal{U}(\mathcal{M}) .
$$

Then $U=\left(u_{v}\right) \in \mathcal{M}^{\omega}, \operatorname{Ad}(U) \in \operatorname{Aut}\left(\mathcal{M}^{\omega}\right)$ is semiliftable, and $\operatorname{Ad}(U)=\alpha$ on $\mathcal{M}$.

An automorphism $\alpha \in \operatorname{Aut}(\mathcal{M})$ is said to be centrally trivial if $\alpha=$ id on $\mathcal{M}_{\omega}$. We denote by $\operatorname{Cnt}(\mathcal{M})$ the set of all centrally trivial automorphisms, which is a normal subgroup of $\operatorname{Aut}(\mathcal{M})$ containing $\operatorname{Int}(\mathcal{M})$. The definition of $\operatorname{Cnt}(\mathcal{M})$ does not depend on the choice of a free ultrafilter $\omega$. An important fact is that $\alpha \notin \operatorname{Cnt}(\mathcal{M})$ if and only if $\alpha$ is free on $\mathcal{M}_{\omega}$ (see $[3,33]$ ). Thus a centrally non-trivial automorphism is often called a centrally free automorphism.

By [5] and [23, Theorem 1],

$$
\operatorname{Ker}(\bmod )=\overline{\operatorname{Int}}(\mathcal{M}), \quad \operatorname{Cnt}_{r}(\mathcal{M})=\operatorname{Cnt}(\mathcal{M})
$$

for an injective factor $\mathcal{M}$, and this characterization of $\overline{\operatorname{Int}}(\mathcal{M})$ and $\operatorname{Cnt}(\mathcal{M})$ plays an essential role in our argument. 
4.2. Ultraproduct and quasi cocycle crossed actions. Let $\alpha$ and $\beta$ be cocycle crossed actions of $G$ on $\mathcal{M}$ with $\operatorname{Inv}(\alpha)=\operatorname{Inv}(\beta)$. Then we get two quasi cocycle crossed actions $\left(\alpha_{\tilde{p}}, c^{a}\right)$ and $\left(\beta_{\tilde{p}}, c^{b}\right)$ with $\bmod \left(\alpha_{\tilde{p}}\right)=\bmod \left(\beta_{\tilde{p}}\right)$, and $\alpha_{\tilde{q}}, \beta_{\tilde{q}} \notin \mathrm{Cnt}_{r}(\mathcal{M})$ for $e \neq q \in Q$. By the characterization of $\overline{\operatorname{Int}}(\mathcal{M})$ and $\mathrm{Cnt}_{r}(\mathcal{M})$, there exists a sequence of unitaries $\left\{u^{v}(\tilde{r})\right\}_{\nu} \subset \mathcal{M}, r \in Q$, such that $\alpha_{\tilde{r}}=\lim _{v} u^{\nu}(\tilde{r}) \circ \beta_{\tilde{r}}$, and $\alpha_{\tilde{r}}$ and $\beta_{\tilde{r}}$ induce free actions of $Q$ on $\mathcal{M}_{\omega}$. Thus we can approximate $\alpha$ by unitary perturbation of $\beta$. However we also need to approximate 2-cocycles to apply the intertwining argument in our setting. This subsection is devoted to solve this problem. Our goal in this section is Lemma 4.6.

Let $\left\{u^{v}(\tilde{r})\right\}_{\mathcal{v}} \subset \mathcal{M}$ be as above, and set $U(\tilde{r})=\left(u^{v}(\tilde{r})\right) \in \mathcal{M}^{\omega}$. We have

$$
\alpha_{\tilde{r}}=\operatorname{Ad}(U(\tilde{r})) \circ \beta_{\tilde{r}}
$$

on $\mathcal{M}$. We want to extend $U(\tilde{r})$ for all $g \in G$ so that $\alpha_{g}=\operatorname{Ad}(U(g)) \circ \beta_{g}$ on $\mathcal{M}$. If $g=n \tilde{r}$, then it is easy to see that the two unitaries

$$
a(n, \tilde{r})^{*} \sigma_{n}(U(\tilde{r})) b(n, \tilde{r})
$$

and

$$
a\left(\tilde{r}, \tilde{r}^{-1} n \tilde{r}\right)^{*} U(\tilde{r}) b\left(\tilde{r}, \tilde{r}^{-1} n \tilde{r}\right)
$$

have the desired property. The following lemma says that these two unitaries coincide.

Lemma 4.1. For any $W(g)=\left(w^{v}(g)\right) \in \mathcal{U}\left(\mathcal{M}^{\omega}\right)$ with $\alpha_{g}=\lim _{v \rightarrow \omega} w^{v}(g) \circ \beta_{g}$, we have

$$
a(n, g)^{*} \sigma_{n}(W(g)) b(n, g)=a\left(g, g^{-1} n g\right)^{*} W(g) b\left(g, g^{-1} n g\right)
$$

for $g \in G$ and $n \in N$.

Proof. It suffices to show that $W(g)^{*} d(a)(n, g) \sigma_{n}(W(g))=d(b)(n, g)$. Since the canonical extension is continuous in the $u$-topology, we have $\lim _{v \rightarrow \omega} \operatorname{Ad}\left(w^{v}(g)\right) \circ \tilde{\beta}_{g}=\tilde{\alpha}_{g}$ in $\operatorname{Aut}(\tilde{\mathcal{M}})$. Then it follows that

$$
\begin{aligned}
\lim _{\nu \rightarrow \omega} w^{\nu}(g)^{*} d(a)(n, g) \sigma_{n}\left(w^{\nu}(g)\right) & =\lim _{\nu \rightarrow \omega} w^{\nu}(g)^{*} d(a)(n, g) \tilde{\sigma}_{n}\left(w^{\nu}(g)\right) \\
& =\lim _{\nu \rightarrow \omega} w^{\nu}(g)^{*} d(a)(n, g) \tilde{u}(n) w^{\nu}(g) \tilde{u}(n)^{*} \\
& =\tilde{\beta}_{g} \circ \tilde{\alpha}_{g}^{-1}(d(a)(n, g) \tilde{u}(n)) \tilde{u}(n)^{*} \\
& =\lambda(n, g)^{*} \tilde{\beta}_{g}\left(\tilde{u}\left(g^{-1} n g\right)\right) \tilde{u}(n)^{*}=d(b)(n, g) .
\end{aligned}
$$

Here, note that $\tilde{\beta}_{g} \circ \tilde{\alpha}_{g}^{-1}(\lambda(n, g))=\lambda(n, g)$, since $\bmod \left(\alpha_{g}\right)=\bmod \left(\beta_{g}\right)$.

Define

$$
\begin{aligned}
U(n \tilde{r}) & =a(n, \tilde{r}) \sigma_{n}(U(\tilde{r})) b(n, \tilde{r})^{*}, \\
\gamma_{g} & =\operatorname{Ad}(U(g)) \circ \beta_{g}, \\
V(g, h) & =U(g) \beta_{g}(U(h)) b(g, h) U(g h)^{*} .
\end{aligned}
$$

Then $(\gamma, V)$ is a cocycle crossed action of $G$ on $\mathcal{M}^{\omega}$ with $\left.\gamma_{g}\right|_{\mathcal{M}}=\alpha_{g}$. Note that $V(m, n)=1$, $m, n \in N$, since $U(n)=1$ for $n \in N$. Our first task is to show that $p \in Q \rightarrow \gamma_{\tilde{p}}$ is a free cocycle crossed action of $Q$ on $\mathcal{M}_{\omega}$. 
Lemma 4.2. For $g \in G$ and $n \in N$, we have $V(n, g)=a(n, g)$ and $V(g, n)=a(g, n)$.

Proof. From the definition of $U(n \tilde{r})$, it follows that

$$
\begin{aligned}
V(n, \tilde{r}) & =\sigma_{n}(U(\tilde{r})) b(n, \tilde{r}) U(n \tilde{r})^{*} \\
& =\sigma_{n}(U(\tilde{r})) b(n, \tilde{r}) b(n, \tilde{r})^{*} \sigma_{n}\left(U(\tilde{r})^{*}\right) a(n, \tilde{r})=a(n, \tilde{r}) .
\end{aligned}
$$

By Lemma 4.1, we have $U(\tilde{r} n)=U\left(\tilde{r} n \tilde{r}^{-1} \tilde{r}\right)=a(\tilde{r}, n)^{*} U(\tilde{r}) b(\tilde{r}, n)$. Hence

$$
\begin{aligned}
V(\tilde{r}, n) & =U(\tilde{r}) b(\tilde{r}, n) U(\tilde{r} n)^{*} \\
& =U(\tilde{r}) b(\tilde{r}, n) b(\tilde{r}, n)^{*} U(\tilde{r})^{*} a(\tilde{r}, n)=a(\tilde{r}, n) .
\end{aligned}
$$

If $g=m \tilde{r}$, then, by the cocycle identity,

$$
\begin{aligned}
V(n, g) & =V(n, m \tilde{r}) \\
& =\sigma_{n}\left(V(m, \tilde{r})^{*}\right) V(n, m) V(n m, \tilde{r}) \\
& =\sigma_{n}\left(a(m, \tilde{r})^{*}\right) a(n m, \tilde{r})=a(n, m \tilde{r})=a(n, g) .
\end{aligned}
$$

In a similar way as above, we can verify $V(\tilde{r} m, n)=a(\tilde{r} m, n)$.

We remark that the conclusion of Lemma 4.2 holds for any $\left(u^{\nu}(\tilde{p})\right) \in \mathcal{M}^{\omega}$ which approximates $\alpha_{\tilde{p}} \circ \beta_{\tilde{p}}^{-1}$. However $V(\tilde{p}, \tilde{q})=a(\tilde{p}, \tilde{q}), p, q \in Q$, does not hold in general. To get this equality, we must choose a suitable $\left(u^{v}(\tilde{p})\right)_{v} \in U\left(\mathcal{M}^{\omega}\right)$.

Lemma 4.3. Put $z(p, q)=U(\tilde{p}) \beta_{\tilde{p}}(U(\tilde{q})) c^{b}(p, q) U(\widetilde{p q})^{*} c^{a}(p, q)^{*}, p, q \in Q$. Then $z(p, q) \in \mathcal{M}_{\omega}$.

Proof. For two sequences of functionals $\left\{\psi_{v}\right\}_{\nu},\left\{\varphi_{\nu}\right\}_{\nu} \subset \mathcal{M}_{*}$, we write $\psi_{\nu} \sim \varphi_{\nu}$ if $\lim _{\nu \rightarrow \omega}\left\|\psi_{\nu}-\varphi_{\nu}\right\|=0$. Then

$$
\begin{aligned}
\alpha_{\tilde{p}} \circ \alpha_{\tilde{q}}(\varphi) & \sim \operatorname{Ad}\left(u^{v}(\tilde{p})\right) \beta_{\tilde{p}}\left(u^{v}(\tilde{q})\right) \circ \beta_{\tilde{p}} \circ \beta_{\tilde{q}}(\varphi) \\
& =\operatorname{Ad}\left(u^{v}(\tilde{p}) \beta_{\tilde{p}}\left(u^{v}(\tilde{q})\right) c^{b}(p, q)\right) \circ \beta_{\widetilde{p q}} \circ \sigma_{m(p, q)}(\varphi) \\
& \sim \operatorname{Ad}\left(u^{v}(\tilde{p}) \beta_{\tilde{p}}\left(u^{v}(\tilde{q})\right) c^{b}(p, q) u^{v}(\widetilde{p q})^{*}\right) \circ \alpha_{\widetilde{p q}} \circ \sigma_{m(p, q)}(\varphi) \\
& =\operatorname{Ad}\left(u^{v}(\tilde{p}) \beta_{\tilde{p}}\left(u^{v}(\tilde{q})\right) c^{b}(p, q) u^{v}(\widetilde{p q})^{*} c^{a}(p, q)^{*}\right) \circ \alpha_{\tilde{p}} \circ \alpha_{\tilde{q}}(\varphi) .
\end{aligned}
$$

This implies $U(\tilde{p}) \beta_{\tilde{p}}(U(\tilde{q})) c^{b}(p, q) U(\widetilde{p q})^{*} c^{a}(p, q)^{*} \in \mathcal{M}_{\omega}$.

Lemma 4.4. $\left(\gamma_{\tilde{p}}, z\right)$ is a cocycle crossed action of $Q$ on $\mathcal{M}_{\omega}$.

Proof. Set

$$
z^{\prime}(p, q)=V(\tilde{p}, \tilde{q}) V(\widetilde{p q}, m(p, q))^{*}=U(\tilde{p}) \beta_{\tilde{p}}(U(\tilde{q})) c^{b}(p, q) U(\widetilde{p q})^{*} .
$$

By Lemma 3.4, $\left(\gamma_{\tilde{p}}, z^{\prime}\right)$ is a quasi cocycle crossed action of $Q$, and $z^{\prime}(p, q)=z(p, q) c^{a}(p, q)$.

For $x \in \mathcal{M}_{\omega}$, we have

$$
\gamma_{\tilde{p}} \circ \gamma_{\tilde{q}}(x)=\operatorname{Ad}\left(z^{\prime}(p, q)\right) \circ \gamma_{\tilde{p q}} \circ \sigma_{m(p, q)}(x)=\operatorname{Ad}(z(p, q)) \circ \gamma_{\widetilde{p q}}(x),
$$

since $\operatorname{Ad}\left(c^{a}(p, q)\right), \sigma_{m(p, q)} \in \operatorname{Cnt}(\mathcal{M})$. 
We next show that $z(\cdot, \cdot)$ satisfies the 2-cocycle identity. By Lemma 3.3, we have

$$
z^{\prime}(p, q) \gamma_{\widetilde{p q}}\left(d(V)(m(p, q), \tilde{r})^{*}\right) z^{\prime}(p q, r)=\gamma_{\tilde{p}}\left(z^{\prime}(q, r)\right) z^{\prime}(p, q r) .
$$

By Lemma 4.2 and $\left.\gamma_{g}\right|_{\mathcal{M}}=\alpha_{g}$, the left-hand side is

$$
\begin{aligned}
& z^{\prime}(p, q) \gamma_{\tilde{p q}}\left(d(V)(m(p, q), \tilde{r})^{*}\right) z^{\prime}(p q, r) \\
& =z(p, q) c^{a}(p, q) \alpha \widetilde{p q}\left(d(a)(m(p, q), \tilde{r})^{*}\right) z(p q, r) c^{a}(p q, r) \\
& =z(p, q) z(p q, r) c^{a}(p, q) \alpha_{\widetilde{p q}}\left(d(a)(m(p, q), \tilde{r})^{*}\right) c^{a}(p q, r) .
\end{aligned}
$$

The right-hand side is

$$
\begin{aligned}
\gamma_{\tilde{p}}\left(z^{\prime}(q, r)\right) z^{\prime}(p, q r) & =\gamma_{\tilde{p}}\left(z(q, r) c^{a}(q, r)\right) z(p, q r) c^{a}(p, q r) \\
& =\gamma_{\tilde{p}}(z(q, r)) z(p, q r) \alpha_{\tilde{p}}\left(c^{a}(q, r)\right) c^{a}(p, q r) .
\end{aligned}
$$

Then Lemma 3.3 yields $z(p, q) z(p q, r)=\gamma_{\tilde{p}}(z(q, r)) z(p, q r)$.

The next step is to replace $U(\tilde{p})$ so that $z(p, q)=1$. To this end, we need the second cohomology vanishing theorem for cocycle crossed actions on $\mathcal{M}_{\omega}$.

Proposition 4.5. Let $Q$ be a discrete amenable group, and $(\gamma, u)$ a semiliftable cocycle crossed action of $Q$ on $\mathcal{M}_{\omega}$. Then $u(\cdot, \cdot)$ is a coboundary. Namely, there exists a map $w(\cdot) \in \operatorname{Map}_{0}\left(Q, U\left(\mathcal{M}_{\omega}\right)\right)$ such that $w(g) \gamma_{g}(w(h)) u(g, h) w(g h)^{*}=1$.

Proposition 4.5 was first proved by Ocneanu [33, Proposition 7.4], and later a more simplified proof was presented in [31, Lemma 4.3] with generalization to the discrete amenable Kac algebra case. For the readers' convenience, we present a proof of Proposition 4.5 based on the argument in [31] in the appendix.

By using Proposition 4.5, we can prove the main result of this section.

Lemma 4.6. Let $\alpha$ and $\beta$ be as above. Then there exists a sequence $\left\{u^{v}(p)\right\}_{v} \subset \mathcal{U}(\mathcal{M})$, $p \in Q$, such that

(1) $\alpha_{\tilde{p}}=\lim _{v \rightarrow \omega} \operatorname{Ad}\left(u^{v}(p)\right) \circ \beta_{\tilde{p}}$ in the u-topology,

(2) $c^{a}(p, q)=\lim _{v \rightarrow \omega} u^{v}(p) \beta_{\tilde{p}}\left(u^{v}(q)\right) c^{b}(p, q) u^{v}(p q)^{*}$ in the $\sigma$-strong* topology.

Proof. Let $\left(\gamma_{\tilde{p}}, z(p, q)\right)$ be as in Lemma 4.4. By Proposition 4.5, there exists a unitary $a(p) \in U\left(\mathcal{M}_{\omega}\right)$ such that $a(p) \gamma_{\tilde{p}}(a(q)) z(p, q) a(p q)^{*}=1$. Then we have

$$
\begin{aligned}
1 & =a(p) \gamma_{\tilde{p}}(a(q)) z(p, q) a(p q)^{*} \\
& =a(p) U(\tilde{p}) \beta_{\tilde{p}}(a(q)) U(\tilde{p})^{*} U(\tilde{p}) \beta_{\tilde{p}}(U(\tilde{q})) c^{b}(p, q) U(\widetilde{p q})^{*} c^{a}(p, q)^{*} a(p q)^{*} \\
& =a(p) U(\tilde{p}) \beta_{\tilde{p}}(a(q) U(\tilde{q})) c^{b}(p, q) U(\widetilde{p q})^{*} a(p q)^{*} c^{a}(p, q)^{*} .
\end{aligned}
$$

Thus the representing sequence of $a(p) U(\tilde{p})$ is a desired one.

The following lemma is not needed in the rest of this paper. However we present it since a similar argument appears in the proof of the main theorem in $\$ 5.2$. 
Lemma 4.7. Choose $U(\tilde{p})=\left(u^{v}(p)\right)_{v} \in \mathcal{M}^{\omega}$ as in Lemma 4.6. Then we have $V(g, h)=a(g, h)$ for all $g, h \in G$.

Proof. By Lemma 4.6, we have

$$
\begin{aligned}
a(\tilde{p}, \tilde{q}) a(\widetilde{p q}, m(p, q))^{*} & =c^{a}(p, q) \\
& =U(\tilde{p}) \beta_{\tilde{p}}(U(\tilde{q})) c^{b}(p, q) U(\widetilde{p q})^{*} \\
& =V(\tilde{p}, \tilde{q}) V(\widetilde{p q}, m(p, q))^{*} .
\end{aligned}
$$

Then by Lemma 4.2, we have $V(\tilde{p}, \tilde{q})=a(\tilde{p}, \tilde{q})$. If $g=m \tilde{p}$, then

$$
\begin{aligned}
V(g, \tilde{q}) & =V(m \tilde{p}, \tilde{q}) \\
& =V(m, \tilde{p})^{*} \sigma_{m}(V(\tilde{p}, \tilde{q})) V(m, \tilde{p} \tilde{q}) \\
& =a(m, \tilde{p})^{*} \sigma_{m}(a(\tilde{p}, \tilde{q})) a(m, \tilde{p} \tilde{q})=a(m \tilde{p}, \tilde{q})=a(g, \tilde{q}) .
\end{aligned}
$$

For $h=n \tilde{q}$, we have

$$
\begin{aligned}
V(g, h) & =V(g, n \tilde{q}) \\
& =\gamma_{g}\left(V(n, \tilde{q})^{*}\right) V(g, n) V(g n, \tilde{q}) \\
& =\alpha_{g}\left(a(n, \tilde{q})^{*}\right) a(g, n) a(g n, \tilde{q})=a(g, n \tilde{q})=a(g, h)
\end{aligned}
$$

by Lemma 4.2 and thus the above result.

4.3. Approximate cohomology vanishing. In what follows, we write $A \Subset B$ if $A$ is a finite subset of $B$.

Let $K$ be a discrete amenable group. Let $F \Subset K$ and $\delta>0$. In this paper, we say that $S \Subset K$ is $(F, \delta)$-invariant if

$$
\left|S \cap \bigcap_{g \in F} g^{-1} S\right|>(1-\delta)|S|
$$

We use the following Rohlin type theorem to show approximate cohomology vanishing.

Theorem 4.8. Let $K$ be a discrete amenable group, and $\gamma_{g}$ a semiliftable, strongly free action of $K$ on $\mathcal{M}_{\omega}$. Fix $e \in F \Subset K, \delta>0$, and let $S \Subset K$ be an $(F, \delta)$-invariant finite set. Then there exists a partition of unity $\left\{E_{S}\right\}_{s \in S} \subset \mathcal{M}_{\omega}$ such that

$$
\begin{gathered}
\sum_{s \in g^{-1} S \cap S}\left\|\gamma_{g}\left(E_{S}\right)-E_{g s}\right\|_{1}<4 \delta^{\frac{1}{2}}, \quad g \in F, \\
\sum_{s \in S \backslash g^{-1} S}\left\|E_{S}\right\|_{1}<3 \delta^{\frac{1}{2}}, \quad g \in F, \\
{\left[\gamma_{g}\left(E_{S}\right), E_{S^{\prime}}\right]=0, \quad g \in F, s, s^{\prime} \in S .}
\end{gathered}
$$

Moreover we can take $\left\{E_{S}\right\}_{s \in S}$ in the relative commutant of any countable subset of $\mathcal{M}_{\omega}$. 
Here an action $\gamma$ is said to be strongly free if $\gamma$ is free on any relative commutant of a countable subset of $\mathcal{M}_{\omega}$. In [33, Lemmas 5.6, 5.7], Ocneanu showed that a centrally free action is strongly free. We call $\left\{E_{s}\right\}_{s \in S}$ in the above theorem Rohlin projections for $\gamma$. Here one of the super natural properties of the ultraproduct appears, i.e., such strong freeness never happens in the "separable world", and this fact forces us to treat nonseparable von Neumann algebras to know separable ones.

The proof of Theorem 4.8 is essentially the same as that of [33, Theorem 6.1]. (See the appendix for details of the proof.) Since we do not need a paving structure of a discrete amenable group, our Rohlin type theorem takes a simpler form than Ocneanu's one.

The following lemma is the key for our intertwining argument in the next section.

Lemma 4.9. Let $K$ be a discrete amenable group and let us assume that $F \Subset K$, $\Psi \Subset \mathcal{M}_{*}, \Phi \Subset\left(\mathcal{M}_{*}\right)_{+}$and $0<\varepsilon<1$ are given. Let $S$ be an $(F, \varepsilon)$-invariant finite set. Let $\gamma \in \operatorname{Map}_{0}(K, \operatorname{Aut}(\mathcal{M}))$ such that $\gamma_{g h} \equiv \gamma_{g} \gamma_{h}$, and $\gamma_{g} \not \equiv$ id for $g \neq e, \operatorname{modulo} \operatorname{Cnt}(\mathcal{M})$. Assume that a family of unitaries $\{u(g)\}_{g \in K} \subset \mathcal{U}(\mathcal{M})$ satisfies

$$
\begin{gathered}
\|[\psi, u(s)]\|<(3|S|)^{-1} \varepsilon, \quad s \in S, \psi \in \Psi, \\
\left\|\varphi \cdot\left(u(g) \gamma_{g}(u(s)) u(g s)^{*}-1\right)\right\|<5 \varepsilon, \\
\left\|\left(u(g) \gamma_{g}(u(s)) u(g s)^{*}-1\right) \cdot \varphi\right\|<5 \varepsilon,
\end{gathered} \quad \varphi \in \Phi, g \in F, s \in S .
$$

Let $\left\{E_{s}\right\}_{s \in S} \subset \mathcal{M}_{\omega}$ be Rohlin projections for $\gamma$ as in Theorem 4.8, and set

$$
W=\sum_{s \in S} u(s) E_{s} \in \mathcal{M}^{\omega} .
$$

Choose a representing sequence $W=\left(w^{v}\right)_{v}$ consisting of unitaries. Then for a sufficiently large $v, w=w^{v}$ satisfies

$$
\begin{gathered}
\|[w, \psi]\|<\varepsilon, \quad \psi \in \Psi, \\
\left\|\left(u(g) \gamma_{g}(w) w^{*}-1\right) \cdot \varphi\right\|<7 \sqrt[4]{\varepsilon}, \quad g \in F, \varphi \in \Phi . \\
\left\|\varphi \cdot\left(u(g) \gamma_{g}(w) w^{*}-1\right)\right\|<7 \sqrt[4]{\varepsilon},
\end{gathered}
$$

Proof. At first we have the following inequalities for a normal state $\varphi$ and $x \in \mathcal{M}$ :

$$
\begin{aligned}
\|x \cdot \varphi\| & \leq \sqrt{\|x\| \varphi(|x|)}, & \|\varphi \cdot x\| & \leq \sqrt{\|x\| \varphi\left(\left|x^{*}\right|\right)}, \\
\varphi(|x|) & \leq\|x \cdot \varphi\|, & \varphi\left(\left|x^{*}\right|\right) & \leq\|\varphi \cdot x\| .
\end{aligned}
$$

Since Theorem 4.8 is stated in terms of $L^{1}$-norm and we have $\varphi(|\cdot|)=\|\cdot\|_{1}$ on $\mathcal{M}_{\omega}$, we first give estimation by $\varphi(|\cdot|)$, and then apply the above inequalities. Note that $\varphi(|\cdot|)$ is not a norm of $\mathcal{M}^{\omega}$, i.e., it is not subadditive. We remark that the advantage of use of the $L^{1}$-norm with respect to $\tau_{\omega}$ is that an equality $\|E+F\|_{1}=\|E\|_{1}+\|F\|_{1}$ holds for orthogonal projections $E, F \in \mathcal{M}_{\omega}$.

Note that $\left|\sum_{i} x_{i} p_{i}\right|=\sum_{i}\left|x_{i}\right| p_{i}$ for $x_{k} \in \mathcal{M}$ and a partition of unity $\left\{p_{i}\right\} \subset \mathcal{M}_{\omega}$, and

$$
\varphi(a b)=\varphi(a) \tau_{\omega}(b), \quad a \in \mathcal{M}, b \in \mathcal{M}_{\omega} .
$$


The former is trivial since $\mathcal{M}_{\omega} \subset \mathcal{M}^{\prime} \cap \mathcal{M}^{\omega}$. To verify the latter equality, take a representing sequence $b=\left(b^{v}\right)$. Since $\lim _{v \rightarrow \omega} b^{v}=\tau_{\omega}(b) \in \mathbb{C}$ in the $\sigma$-weak topology,

$$
\varphi(a b)=\lim _{\nu \rightarrow \omega} \varphi\left(a b^{\nu}\right)=\varphi\left(a \tau_{\omega}(b)\right)=\varphi(a) \tau_{\omega}(b) .
$$

Let $W$ be as in the statement of Lemma 4.9. Then we have

$$
\begin{aligned}
\varphi\left(\left|u(g) \gamma_{g}(W) W^{*}-1\right|\right) & =\varphi\left(\left|\sum_{h, k \in S}\left(u(g) \gamma_{g}(u(h)) u(k)^{*}-1\right) \gamma_{g}\left(E_{h}\right) E_{k}\right|\right) \\
& =\sum_{h, k \in S} \varphi\left(\left|u(g) \gamma_{g}(u(h)) u(k)^{*}-1\right| \gamma_{g}\left(E_{h}\right) E_{k}\right) \\
& =\sum_{h, k \in S} \varphi\left(\left|u(g) \gamma_{g}(u(h)) u(k)^{*}-1\right|\right)\left\|\gamma_{g}\left(E_{h}\right) E_{k}\right\|_{1} .
\end{aligned}
$$

We divide $\sum_{h, k \in S}$ into three parts as follows:

$$
\sum_{h, k \in S}=\sum_{\substack{h \in S \cap g^{-1} S \\ k \in S}}+\sum_{\substack{h \in S \backslash g^{-1} S \\ k \in S}}=\sum_{\substack{h \in S \cap g^{-1} S \\ g h=k \in S}}+\sum_{\substack{h \in S \cap g^{-1} S \\ g h \neq k \in S}}+\sum_{\substack{h \in S \backslash g^{-1} S \\ k \in S}} .
$$

Since $\varphi(|x|) \leq\|x \cdot \varphi\|$, the first term is less than $5 \varepsilon$ as seen below:

$$
\begin{aligned}
& \sum_{\substack{h \in S \cap g^{-1} S \\
g h=k \in S}} \varphi\left(\left|u(g) \gamma_{g}(u(h)) u(k)^{*}-1\right|\right)\left\|\gamma_{g}\left(E_{h}\right) E_{k}\right\|_{1} \\
& \quad=\sum_{h \in S \cap g^{-1} S} \varphi\left(\left|u(g) \gamma_{g}(u(h)) u(g h)^{*}-1\right|\right)\left\|\gamma_{g}\left(E_{h}\right) E_{g h}\right\|_{1} \\
& \quad \leq 5 \varepsilon \sum_{h \in S \cap g^{-1} S}\left\|\gamma_{g}\left(E_{h}\right) E_{g h}\right\|_{1} \leq 5 \varepsilon .
\end{aligned}
$$

The second term is estimated as follows:

$$
\begin{aligned}
& \sum_{\substack{h \in S \cap g^{-1} S \\
k \in S, k \neq g h}} \varphi\left(\left|\left(u(g) \gamma_{g}(u(h)) u(k)^{*}-1\right)\right|\right)\left\|\gamma_{g}\left(E_{h}\right) E_{k}\right\|_{1} \\
& \leq 2 \sum_{h \in S \cap g^{-1} S}\left\|\left(1-E_{g h}\right) \gamma_{g}\left(E_{h}\right)\right\|_{1} \\
& \quad=2 \sum_{h \in S \cap g^{-1} S}\left\|\left(1-E_{g h}\right)\left(\gamma_{g}\left(E_{h}\right)-E_{g h}\right)\right\|_{1}<8 \sqrt{\varepsilon} .
\end{aligned}
$$

The third term is estimated as follows:

$$
\sum_{\substack{h \in S \backslash g^{-1} S \\ k \in S}} \varphi\left(\left|u(g) \gamma_{g}(u(h)) u(k)^{*}-1\right|\right)\left\|\gamma_{g}\left(E_{h}\right) E_{k}\right\|_{1} \leq \sum_{h \in S \backslash g^{-1} S} 2\left|\gamma_{g}\left(E_{h}\right)\right|<6 \sqrt{\varepsilon} .
$$

Since $0<\varepsilon<1$, we get

$$
\varphi\left(\left|u(g) \gamma_{g}(W) W^{*}-1\right|\right)<5 \varepsilon+8 \sqrt{\varepsilon}+6 \sqrt{\varepsilon}<19 \sqrt{\varepsilon}
$$


for $g \in F$. In the same way, we have

$$
\varphi\left(\left|\left(u(g) \gamma_{g}(W) W^{*}-1\right)^{*}\right|\right)<19 \sqrt{\varepsilon}, \quad g \in F .
$$

Let $W=\left(w^{v}\right)_{v}$ and $E_{s}=\left(e_{s}^{v}\right)_{\nu}$ be representing sequences consisting of unitaries and projections, respectively. Set $a^{v}=\sum_{s \in S} u(s) e_{s}^{v}$. Note that $a^{v}$ is not a unitary in general. Since $\left(a^{v}\right)=\left(w^{v}\right)$ in $\mathcal{M}^{\omega},\left\{a^{v}-w^{\nu}\right\}_{v}$ converges to 0 in the $\sigma$-strong* topology as $v \rightarrow \omega$.

Fix sufficiently large $v$ such that

$$
\begin{gathered}
\left\|\left[\psi, w^{\nu}-a^{\nu}\right]\right\|<\frac{\varepsilon}{3}, \quad\left\|\left[\psi, e_{s}^{\nu}\right]\right\|<\frac{\varepsilon}{3|S|}, \quad \psi \in \Psi, s \in S, \\
\varphi\left(\left|u(g) \gamma_{g}\left(w^{\nu}\right) w^{\nu *}-1\right|\right)<19 \sqrt{\varepsilon}, \quad g \in F, \varphi \in \Phi . \\
\varphi\left(\left|\left(u(g) \gamma_{g}\left(w^{\nu}\right) w^{\nu *}-1\right)^{*}\right|\right)<19 \sqrt{\varepsilon},
\end{gathered}
$$

It follows that

$$
\begin{aligned}
\left\|\left[\psi, w^{\nu}\right]\right\| & \leq\left\|\left[\psi, w^{\nu}-a^{\nu}\right]\right\|+\left\|\left[\psi, a^{\nu}\right]\right\|<\frac{\varepsilon}{3}+\left\|\left[\psi, \sum_{k \in S} u(k) e_{k}^{\nu}\right]\right\| \\
& \leq \frac{\varepsilon}{3}+\sum_{k \in S}\left(\left\|[\psi, u(k)] e_{k}^{v}\right\|+\left\|u(k)\left[\psi, e_{k}^{\nu}\right]\right\|\right)<\varepsilon .
\end{aligned}
$$

By the inequality presented in the beginning of the proof, $w=w^{v}$ is a desired unitary.

\section{Intertwining argument and the proof of the main theorem}

In this section, we present a proof of the main theorem, Theorem 2.3 (2).

5.1. Classification of quasi cocycle actions. We can assume that $\beta$ is an action, i.e., $b(g, h)=1, g, h \in G$, due to the existence of model actions. Recall that we assumed that $\sigma_{n}=\alpha_{n}=\beta_{n}$ is an action of $N$, and fixed a unitary $\tilde{u}(m) \in \tilde{\mathcal{M}}$ with $\tilde{\sigma}_{m}=\operatorname{Ad}(\tilde{u}(m))$ in $\S 3$.

In this subsection, we will classify two quasi cocycle crossed actions $\left(\alpha, c^{a}\right)$ and $(\beta, 1)$ of $Q$. For simplicity, we write $\alpha_{\tilde{p}}$ as $\alpha_{p}$ until the end of the proof of Theorem 5.1.

Theorem 5.1. Let $\left(\alpha, c^{a}\right)$ and $(\beta, 1)$ be as above. Then there exist $\bar{\theta}_{0}, \bar{\theta}_{1} \in \overline{\operatorname{Int}}(\mathcal{M})$ and $\hat{u}^{0}, \hat{u}^{1} \in \operatorname{Map}_{0}(Q, \mathcal{U}(\mathcal{M}))$ such that

$$
\operatorname{Ad}\left(\hat{u}^{0}(p)\right) \circ \bar{\theta}_{0} \circ \alpha_{p} \circ \bar{\theta}_{0}^{-1}=\operatorname{Ad}\left(\hat{u}^{1}(p)\right) \circ \bar{\theta}_{1} \circ \beta_{p} \circ \bar{\theta}_{1}^{-1},
$$

and

$$
\begin{gathered}
\hat{u}^{0}(p) \bar{\theta}_{0} \circ \alpha_{p} \circ \bar{\theta}_{0}^{-1}\left(\hat{u}^{0}(q)\right) \bar{\theta}_{0}\left(c^{\alpha}(p, q)\right) \bar{\theta}_{0} \circ \alpha_{p q} \circ \bar{\theta}_{0}^{-1}\left(\bar{\theta}_{0}(\tilde{u}(m(p, q))) \tilde{u}(m(p, q))^{*}\right) \hat{u}^{0}(p q)^{*} \\
=\hat{u}^{1}(p) \bar{\theta}_{1} \circ \beta_{p} \circ \bar{\theta}_{1}^{-1}\left(\hat{u}^{1}(q)\right) \bar{\theta}_{1} \circ \beta_{p q} \circ \bar{\theta}_{1}^{-1}\left(\bar{\theta}_{1}(\tilde{u}(m(p, q))) \tilde{u}(m(p, q))^{*}\right) \hat{u}^{1}(p q)^{*}
\end{gathered}
$$

We remark that $\bar{\theta}_{i}(\tilde{u}(m(p, q))) \tilde{u}(m(p, q))^{*}, i=0,1$, are indeed in $\mathcal{M}=\tilde{\mathcal{M}}^{\theta}$. 
We will present the proof of Theorem 5.1 by showing the series of lemmas below. Put $\varepsilon_{n}=4^{-n}, n \in \mathbb{N}$. (Until the end of the proof, we use the letter $n$ to denote elements in $\mathbb{N}$.) Fix $F_{n} \Subset Q$ and an $\left(F_{n}, \varepsilon_{n}\right)$-invariant set $S_{n} \Subset Q$ such that

$$
e \in F_{1}, \quad F_{n} \subset F_{n+1}, \quad \bigcup_{n=1}^{\infty} F_{n}=Q, \quad F_{n} \subset S_{n}, \quad F_{n} S_{n} \subset F_{n+1} .
$$

Fix a faithful normal state $\varphi_{0}$. Let $\left\{\Psi_{n}\right\}_{n}$ be an increasing sequence of finite sets of $\mathcal{M}_{*}$ such that $\bigcup_{n} \Psi_{n}$ is total in $\mathcal{M}_{*}$.

Lemma 5.2. Set

$$
\gamma^{(0)}=\alpha, \quad c^{0}(p, q)=c^{a}(p, q), \quad \gamma^{(-1)}=\beta, \quad c^{-1}(p, q)=1 .
$$

For $n \in \mathbb{N}$, there exist

- a family of quasi cocycle crossed actions $\left(\gamma^{(n)}, c^{n}\right)$,

- $u^{n}(\cdot), a^{n}(\cdot), b^{n}(\cdot) \in \operatorname{Map}_{0}(Q, U(\mathcal{M}))$,

- $w_{n} \in \mathcal{U}(\mathcal{M})$,

- an automorphism $\theta_{n} \in \operatorname{Aut}(\mathcal{M})$,

- finite sets $\Phi_{n} \Subset\left(\mathcal{M}_{*}\right)_{+}, \Psi_{n}^{\prime}, \Phi_{n}^{\prime} \Subset \mathcal{M}_{*}$,

satisfying the following conditions:

$$
\begin{aligned}
& \Phi_{n}=\left\{\operatorname{Ad}\left(b^{n-1}(p)\right)\left(\varphi_{0}\right)\right\}_{p \in F_{n}}, \\
& \Phi_{n}^{\prime}=\bigcup_{\substack{p, q \in F_{n} \\
r \in S_{n}, \varphi \in \Phi_{n}}}\left(\gamma_{p}^{(n-1)}\right)^{-1}\left(\left\{\varphi \cdot c^{n-1}(q, r), c^{n-1}(q, r) \cdot \varphi\right\}\right),
\end{aligned}
$$

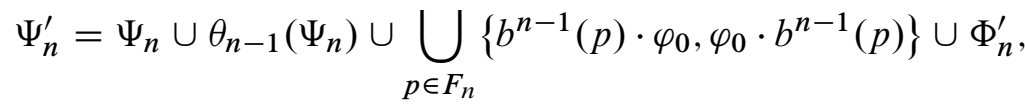

$$
\begin{aligned}
& a^{n}(p)=u^{n}(p) \gamma_{p}^{(n-2)}\left(w_{n}\right) w_{n}^{*}, \quad b^{n}(p)=a^{n}(p) w_{n} b^{n-2}(p) w_{n}^{*}, \\
& \theta_{n}=\operatorname{Ad}\left(w_{n}\right) \circ \theta_{n-2}, \quad n \geq 3,
\end{aligned}
$$

$$
\gamma_{p}^{(n)}=\operatorname{Ad}\left(u^{n}(p)\right) \circ \gamma_{p}^{(n-2)}=\operatorname{Ad}\left(a^{n}(p)\right) \circ \operatorname{Ad}\left(w_{n}\right) \circ \gamma_{p}^{(n-2)} \circ \operatorname{Ad}\left(w_{n}^{*}\right),
$$

(n.5) $c^{n}(p, q)=u^{n}(p) \gamma_{p}^{(n-2)}\left(u^{n}(q)\right) c^{n-2}(p, q) u^{n}(p q)^{*}$,

$$
\left\|\gamma_{p}^{(n)}(\psi)-\gamma_{p}^{(n-1)}(\psi)\right\|<\frac{\varepsilon_{n}}{6\left|S_{n}\right|}, \quad p \in F_{n+1},
$$$$
\psi \in \bigcup_{q \in F_{n+1}}\left(\gamma_{q}^{(n-2)}\right)^{-1}\left(\Psi_{n-1}^{\prime}\right) \cup\left(\gamma_{q}^{(n-1)}\right)^{-1}\left(\Psi_{n}^{\prime}\right),
$$

$$
\left\{\begin{array}{l}
\left\|\varphi \cdot\left(c^{n}(p, q)-c^{n-1}(p, q)\right)\right\|<\frac{\varepsilon_{n}}{2}, \\
\left\|\left(c^{n}(p, q)-c^{n-1}(p, q)\right) \cdot \varphi\right\|<\frac{\varepsilon_{n}}{2},
\end{array} \quad \varphi \in \Phi_{n} \cup \Phi_{n-1}, p \in F_{n}, q \in S_{n},\right.
$$
$\left\{\begin{array}{l}\left\|\varphi \cdot\left(a^{n}(p)-1\right)\right\|<7 \sqrt[4]{\varepsilon_{n-1}}, \\ \left\|\left(a^{n}(p)-1\right) \cdot \varphi\right\|<7 \sqrt[4]{\varepsilon_{n-1}},\end{array} \quad \varphi \in \Phi_{n-1}, p \in F_{n-1}, n \geq 2\right.$,

$$
\left\|\left[w_{n}, \psi\right]\right\|<\varepsilon_{n-1}, \quad \psi \in \Psi_{n-1}^{\prime}, n \geq 2 \text {. }
$$


Proof. We will construct the desired objects by induction.

Step 1. Define $\Phi_{1}, \Phi_{1}^{\prime}$ and $\Psi_{1}^{\prime}$ as in (1.1) and (1.2). (Here we set $b^{0}(p)=1, \theta_{0}=\mathrm{id}$ and $\Psi_{0}=\Phi_{0}=\emptyset$.) By Lemma 4.6, there exists a unitary $u^{1}(p)$ such that

$$
\begin{gathered}
\left\|\operatorname{Ad}\left(u^{1}(p)\right) \circ \gamma_{p}^{(-1)}(\psi)-\gamma_{p}^{(0)}(\psi)\right\|<\frac{\varepsilon_{1}}{6\left|S_{1}\right|}, \\
p \in F_{2}, \psi \in \bigcup_{r \in F_{2}}\left(\gamma_{r}^{(0)}\right)^{-1}\left(\Psi_{1}^{\prime}\right), \\
\left\|\varphi \cdot\left(u^{1}(p) \gamma_{p}^{(-1)}\left(u^{1}(q)\right) u^{1}(p q)^{*}-c^{0}(p, q)\right)\right\|<\frac{\varepsilon_{1}}{2}, \\
\left\|\left(u^{1}(p) \gamma_{p}^{(-1)}\left(u^{1}(q)\right) u^{1}(p q)^{*}-c^{0}(p, q)\right) \cdot \varphi\right\|<\frac{\varepsilon_{1}}{2}, \\
p \in F_{1}, q \in S_{1}, \varphi \in \Phi_{1} .
\end{gathered}
$$

Set

$$
\begin{gathered}
w_{1}=1, \quad u^{1}(p)=a^{1}(p)=u^{1}(p), \quad \theta_{1}=\operatorname{Ad}\left(w_{1}\right), \\
\gamma_{p}^{(1)}=\operatorname{Ad}\left(u^{1}(p)\right) \circ \gamma_{p}^{(-1)}, \quad c^{1}(p, q)=u^{1}(p) \gamma_{p}^{(-1)}(u(q)) u^{1}(p q)^{*} .
\end{gathered}
$$

Then we obtain (1.3), (1.4) and (1.5). The conditions (1.6) and (1.7) follow from (1.a) and (1.b), and the first step is complete. such that

Step 2. Define $\Phi_{2}, \Phi_{2}^{\prime}$ and $\Psi_{2}^{\prime}$ as in (2.1), (2.2). By Lemma 4.6, there exists a unitary $u_{p}^{2}$

$$
\begin{aligned}
& \left\|\operatorname{Ad}\left(u^{2}(p)\right) \circ \gamma_{p}^{(0)}(\psi)-\gamma_{p}^{(1)}(\psi)\right\|<\frac{\varepsilon_{2}}{6\left|S_{2}\right|} \\
& \qquad p \in F_{3}, \psi \in \bigcup_{q \in F_{3}}\left(\gamma_{q}^{(0)}\right)^{-1}\left(\Psi_{1}^{\prime}\right) \cup\left(\gamma_{q}^{(1)}\right)^{-1}\left(\Psi_{2}^{\prime}\right) .
\end{aligned}
$$

$$
\begin{gathered}
\left\|\varphi \cdot\left(u^{2}(p) \gamma_{p}^{(0)}\left(u^{2}(q)\right) c^{0}(p, q) u^{2}(p q)^{*}-c^{1}(p, q)\right)\right\|<\frac{\varepsilon_{2}}{2}, \\
\left\|\left(u^{2}(p) \gamma_{p}^{(0)}\left(u^{2}(q)\right) c^{0}(p, q) u^{2}(p q)^{*}-c^{1}(p, q)\right) \cdot \varphi\right\|<\frac{\varepsilon_{2}}{2}, \\
p \in F_{2}, q \in S_{2}, \varphi \in \Phi_{2} \cup \Phi_{1} .
\end{gathered}
$$

By (2.a) and (1.6), we get

$$
\left\|\operatorname{Ad}\left(u^{2}(p)\right) \circ \gamma_{p}^{(0)}(\psi)-\gamma_{p}^{(0)}(\psi)\right\|<\frac{\varepsilon_{1}}{3\left|S_{1}\right|}, \quad \psi \in \bigcup_{q \in F_{2}}\left(\gamma_{q}^{(0)}\right)^{-1}\left(\Psi_{1}^{\prime}\right), p \in F_{2}
$$

Hence

$$
\left\|\left[u^{2}(p), \psi\right]\right\|<\frac{\varepsilon_{1}}{3\left|S_{1}\right|}, \quad \psi \in \Psi_{1}^{\prime}, p \in F_{2} .
$$

By (2.b) and (1.7), we have

$$
\begin{gathered}
\left\|\varphi \cdot\left(u^{2}(p) \gamma_{p}^{(0)}\left(u^{2}(q)\right) c^{0}(p, q) u^{2}(p q)^{*}-c^{0}(p, q)\right)\right\|<\varepsilon_{1}, \\
\left\|\left(u^{2}(p) \gamma_{p}^{(0)}\left(u^{2}(q)\right) c^{0}(p, q) u^{2}(p q)^{*}-c^{0}(p, q)\right) \cdot \varphi\right\|<\varepsilon_{1}
\end{gathered}
$$

for $p \in F_{1}, q \in S_{1}$ and $\varphi \in \Phi_{1}$. 
Since $\Phi_{1} \cdot c^{0}(p, q) \subset \Psi_{1}^{\prime}, p \in F_{1}, q \in S_{1}$, and $F_{1} S_{1} \subset F_{2}$, we have

$$
\begin{aligned}
\| \varphi \cdot & \left(u^{2}(p) \gamma_{p}^{(0)}\left(u^{2}(q)\right) u^{2}(p q)^{*}-1\right) \| \\
& =\left\|\varphi \cdot u^{2}(p) \gamma_{p}^{(0)}\left(u^{2}(q)\right)-\varphi \cdot u^{2}(p q)\right\| \\
& \leq \varepsilon_{1}+\left\|\varphi \cdot u^{2}(p) \gamma_{p}^{(0)}\left(u^{2}(q)\right)-u^{2}(p q) \cdot \varphi\right\| \\
& =\varepsilon_{1}+\left\|\varphi \cdot u^{2}(p) \gamma_{p}^{(0)}\left(u^{2}(q)\right) c^{0}(p, q)-u^{2}(p q) \cdot \varphi \cdot c^{0}(p, q)\right\| \\
& \leq 2 \varepsilon_{1}+\left\|\varphi \cdot u^{2}(p) \gamma_{p}^{(0)}\left(u^{2}(q)\right) c^{0}(p, q)-\varphi \cdot c^{0}(p, q) u^{2}(p q)\right\| \\
& =2 \varepsilon_{1}+\left\|\varphi \cdot u^{2}(p) \gamma_{p}^{(0)}\left(u^{2}(q)\right) c^{0}(p, q) u^{2}(p q)^{*}-\varphi \cdot c^{0}(p, q)\right\| \\
& <3 \varepsilon_{1}
\end{aligned}
$$

for $\varphi \in \Phi_{1}, p \in F_{1}$ and $q \in S_{1}$.

Since $\left(\gamma_{p}^{(0)}\right)^{-1}\left(c^{0}\left(p^{\prime}, q\right) \cdot \varphi\right) \in \Psi_{1}^{\prime}$ for $p, p^{\prime} \in F_{1}, q \in S_{1}$ and $\varphi \in \Phi_{1}$, it follows that

$$
\left\|\left[\gamma_{p}^{(0)}\left(u^{2}(q)\right), c^{0}\left(p^{\prime}, q^{\prime}\right) \cdot \varphi\right]\right\|=\left\|\left[u^{2}(q),\left(\gamma_{p}^{(0)}\right)^{-1}\left(c^{0}\left(p^{\prime}, q^{\prime}\right) \cdot \varphi\right)\right]\right\|<\varepsilon_{1}
$$

for $p \in F_{1}, q, q^{\prime} \in S_{1}$ and $\varphi \in \Phi_{1}$. Thus

$$
\begin{aligned}
& \left\|\left(u^{2}(p) \gamma_{p}^{(0)}\left(u^{2}(q)\right) u^{2}(p q)^{*}-1\right) \cdot \varphi\right\| \\
& =\left\|\gamma_{p}^{(0)}\left(u^{2}(q)^{*}\right) u^{2}(p)^{*} \cdot \varphi-u^{2}(p q)^{*} \cdot \varphi\right\| \\
& \leq\left\|\left[\gamma_{p}^{(0)}\left(u^{2}(q)^{*}\right) u^{2}(p)^{*}, \varphi\right]\right\|+\left\|\varphi \cdot \gamma_{p}^{(0)}\left(u^{2}(q)^{*}\right) u^{2}(p)^{*}-u^{2}(p q)^{*} \cdot \varphi\right\| \\
& \leq\left\|\gamma_{p}^{(0)}\left(u^{2}(q)^{*}\right)\left[u^{2}(p)^{*}, \varphi\right]\right\|+\left\|\left[\gamma_{p}^{(0)}\left(u^{2}(q)^{*}\right), \varphi\right] u^{2}(p)^{*}\right\| \\
& \quad+\left\|\varphi \cdot \gamma_{p}^{(0)}\left(u^{2}(q)^{*}\right) u^{2}(p)^{*}-u^{2}(p q)^{*} \cdot \varphi\right\| \\
& \leq 2 \varepsilon_{1}+\left\|\varphi \cdot \gamma_{p}^{(0)}\left(u^{2}(q)^{*}\right) u^{2}(p)^{*}-u^{2}(p q)^{*} \cdot \varphi\right\| \\
& =2 \varepsilon_{1}+\left\|c^{0}(p, q) \cdot \varphi \cdot \gamma_{p}^{(0)}\left(u^{2}(q)^{*}\right) u^{2}(p)^{*}-c^{0}(p, q) u^{2}(p q)^{*} \cdot \varphi\right\| \\
& \leq 2 \varepsilon_{1}+\left\|\left[c^{0}(p, q) \cdot \varphi, \gamma_{p}^{(0)}\left(u^{2}(q)^{*}\right) u^{2}(p)^{*}\right]\right\| \\
& \quad+\left\|\gamma_{p}^{(0)}\left(u^{2}(q)^{*}\right) u^{2}(p)^{*} \cdot c^{0}(p, q) \cdot \varphi-c^{0}(p, q) u^{2}(p q)^{*} \cdot \varphi\right\| \\
& \leq 2 \varepsilon_{1}+\left\|\left[c^{0}(p, q) \cdot \varphi, \gamma_{p}^{(0)}\left(u^{2}(q)^{*}\right)\right] u^{2}(p)^{*}\right\| \\
& \quad+\left\|\gamma_{p}^{(0)}\left(u^{2}(q)^{*}\right)\left[c^{0}(p, q) \cdot \varphi, u^{2}(p)^{*}\right]\right\| \\
& \quad+\left\|\gamma_{p}^{(0)}\left(u^{2}(q)^{*}\right) u^{2}(p)^{*} \cdot c^{0}(p, q) \cdot \varphi-c^{0}(p, q) u^{2}(p q)^{*} \cdot \varphi\right\| \\
& \leq 4 \varepsilon_{1}+\left\|\gamma_{p}^{(0)}\left(u^{2}(q)^{*}\right) u^{2}(p)^{*} c^{0}(p, q) \cdot \varphi-c^{0}(p, q) u^{2}(p q)^{*} \cdot \varphi\right\| \\
& =4 \varepsilon_{1}+\left\|\left(u^{2}(p) \gamma_{p}^{(0)}\left(u^{2}(q)\right) c^{0}(p, q) u^{2}(p q)^{*}-c^{0}(p, q)\right) \cdot \varphi\right\| \\
& <5 \varepsilon_{1}
\end{aligned}
$$

holds for $p \in F_{1}, q \in S_{1}$ and $\varphi \in \Phi$.

By Lemma 4.9, there exists a unitary $w_{2}$ such that

$$
\begin{aligned}
\left\|\left(u^{2}(p) \gamma_{p}^{(0)}\left(w_{2}\right) w_{2}^{*}-1\right) \cdot \varphi\right\| & <7 \sqrt[4]{\varepsilon_{1}}, \quad \\
\left\|\varphi \cdot\left(u^{2}(p) \gamma_{p}^{(0)}\left(w_{2}\right) w_{2}^{*}-1\right)\right\| & <7 \sqrt[4]{\varepsilon_{1}}, \\
\left\|\left[w_{2}, \psi\right]\right\| & <\varepsilon_{1}, \quad \psi \in F_{1}, \varphi
\end{aligned}
$$

Put $a^{2}(p)=u^{2}(p) \gamma_{p}^{(0)}\left(w_{2}\right) w_{2}^{*}$, and we obtain (2.8) and (2.9). 
Set

$$
\begin{gathered}
\theta_{2}=\operatorname{Ad}\left(w_{2}\right), \quad c^{2}(p, q)=u^{2}(p) \gamma_{p}^{(0)}\left(u^{2}(q)\right) c^{0}(p, q) u^{2}(p q)^{*}, \\
\gamma_{p}^{(2)}=\operatorname{Ad}\left(u^{2}(p)\right) \circ \gamma_{p}^{(0)}=\operatorname{Ad}\left(a^{2}(p)\right) \circ \theta_{2} \circ \gamma_{p}^{(0)} \circ \theta_{2}^{-1} .
\end{gathered}
$$

Then we obtain (2.3), (2.4) and (2.5). The conditions (2.6) and (2.7) follow from (2.a) and (2.b), and the second step is complete.

Suppose we have constructed the desired objects up to the $(n-1)$-st step.

Step $n$. Define $\Phi_{n}, \Phi_{n}^{\prime}$ and $\Psi_{n}^{\prime}$ as in (n.1) and (n.2). By Lemma 4.6, there exists a unitary $u^{n}(p)$ such that

$$
\begin{aligned}
\| \operatorname{Ad}\left(u^{n}(p)\right) & \circ \gamma_{p}^{(n-2)}(\psi)-\gamma_{p}^{(n-1)}(\psi) \|<\frac{\varepsilon_{n}}{6\left|S_{n}\right|}, \\
& p \in F_{n+1}, \psi \in \bigcup_{q \in F_{n+1}}\left(\gamma_{q}^{(n-2)}\right)^{-1}\left(\Psi_{n-1}^{\prime}\right) \cup\left(\gamma_{q}^{(n-1)}\right)^{-1}\left(\Psi_{n}^{\prime}\right),
\end{aligned}
$$

(n.b)

$$
\begin{gathered}
\left\|\varphi \cdot\left(u^{n}(p) \gamma_{p}^{(n-2)}\left(u^{n}(q)\right) c^{n-2}(p, q) u^{n}(p q)^{*}-c^{n-1}(p, q)\right)\right\|<\frac{\varepsilon_{n}}{2}, \\
\left\|\left(u^{n}(p) \gamma_{p}^{(n-2)}\left(u^{n}(q)\right) c^{n-2}(p, q) u^{n}(p q)^{*}-c^{n-1}(p, q)\right) \cdot \varphi\right\|<\frac{\varepsilon_{n}}{2}, \\
p \in F_{n}, q \in S_{n}, \varphi \in \Phi_{n} \cup \Phi_{n-1} .
\end{gathered}
$$

By (n.a) and $(n-1.6)$, we get

$$
\left\|\operatorname{Ad}\left(u^{n}(p)\right) \circ \gamma_{p}^{(n-2)}(\psi)-\gamma_{p}^{(n-2)}(\psi)\right\|<\frac{\varepsilon_{n-1}}{3\left|S_{n-1}\right|}, \quad \psi \in \bigcup_{q \in F_{n}}\left(\gamma_{q}^{(n-2)}\right)^{-1}\left(\Psi_{n-1}^{\prime}\right), p \in F_{n} .
$$

Hence

$$
\left\|\left[u^{n}(p), \psi\right]\right\|<\frac{\varepsilon_{n-1}}{3\left|S_{n-1}\right|}, \quad \psi \in \Psi_{n-1}^{\prime}, p \in F_{n} .
$$

By $(n . b)$ and $(n-1.7)$, we have

$$
\begin{gathered}
\left\|\varphi \cdot\left(u^{n}(p) \gamma_{p}^{(n-2)}\left(u^{n}(q)\right) c^{n-2}(p, q) u^{n}(p q)^{*}-c^{n-2}(p, q)\right)\right\|<\varepsilon_{n-1}, \\
\left\|\left(u^{n}(p) \gamma_{p}^{(n-2)}\left(u^{n}(q)\right) c^{n-2}(p, q) u^{n}(p q)^{*}-c^{n-2}(p, q)\right) \cdot \varphi\right\|<\varepsilon_{n-1}
\end{gathered}
$$

for $p \in F_{n-1}, q \in S_{n-1}$ and $\varphi \in \Phi_{n-1}$. Since $\Phi_{n-1} \cdot c^{n-2}(p, q) \subset \Psi_{n-1}^{\prime}, p \in F_{n-1}$, $q \in S_{n-1}$, and $F_{n-1} S_{n-1} \subset F_{n}$, we have

$$
\begin{aligned}
\left\|\varphi \cdot\left(u^{n}(p) \gamma_{p}^{(n-2)}\left(u^{n}(q)\right) u^{n}(p q)^{*}-1\right)\right\| \\
\quad=\left\|\varphi \cdot u^{n}(p) \gamma_{p}^{(n-1)}\left(u^{n}(q)\right)-\varphi \cdot u^{n}(p q)\right\| \\
\leq\left\|\left[u^{n}(p q), \varphi\right]\right\|+\left\|\varphi \cdot u^{n}(p) \gamma_{p}^{(n-2)}\left(u^{n}(q)\right)-u^{n}(p q) \cdot \varphi\right\| \\
=\varepsilon_{n-1}+\left\|\varphi \cdot u^{n}(p) \gamma_{p}^{(n-2)}\left(u^{n}(q)\right) c^{n-2}(p, q)-u^{n}(p q) \cdot \varphi \cdot c^{n-2}(p, q)\right\| \\
\leq \varepsilon_{n-1}+\left\|\left[u^{n}(p q), \varphi \cdot c^{n-2}(p, q)\right]\right\| \\
\quad \quad+\left\|\varphi \cdot u^{n}(p) \gamma_{p}^{(n-2)}\left(u^{n}(q)\right) c^{n-2}(p, q)-\varphi \cdot c^{n-2}(p, q) u^{n}(p q)\right\| \\
\leq 2 \varepsilon_{n-1}+\left\|\varphi \cdot u^{n}(p) \gamma_{p}^{(n-2)}\left(u^{n}(q)\right) c^{n-2}(p, q) u^{n}(p q)^{*}-\varphi \cdot c^{n-2}(p, q)\right\|<3 \varepsilon_{n-1}
\end{aligned}
$$

for $\varphi \in \Phi_{n-1}, p \in F_{n-1}$ and $q \in S_{n-1}$. 
we have

Since $\left(\gamma_{p}^{(n-2)}\right)^{-1}\left(c^{n-2}\left(p^{\prime}, q\right) \cdot \varphi\right) \in \Psi_{n-1}^{\prime}$ for $p, p^{\prime} \in F_{n-1}, q \in S_{n-1}$ and $\varphi \in \Phi_{n-1}$,

$$
\left\|\left[\gamma_{p}^{(n-2)}\left(u^{n}(q)\right), c^{n-2}\left(p^{\prime}, q^{\prime}\right) \cdot \varphi\right]\right\|=\left\|\left[u^{n}(q),\left(\gamma_{p}^{(n-2)}\right)^{-1}\left(c^{n-2}\left(p^{\prime}, q^{\prime}\right) \cdot \varphi\right)\right]\right\|<\varepsilon_{n-1}
$$

for $p, p^{\prime} \in F_{n-1}, q, q^{\prime} \in S_{n-1}$ and $\varphi \in \Phi_{n-1}$. Thus

$$
\begin{aligned}
& \left\|\left(u^{n}(p) \gamma_{p}^{(n-2)}\left(u^{n}(q)\right) u^{n}(p q)^{*}-1\right) \cdot \varphi\right\| \\
& =\left\|\gamma_{p}^{(n-2)}\left(u^{n}(q)^{*}\right) u^{n}(p)^{*} \cdot \varphi-u^{n}(p q)^{*} \cdot \varphi\right\| \\
& \leq\left\|\left[\gamma_{p}^{(n-2)}\left(u^{n}(q)^{*}\right) u^{n}(p)^{*}, \varphi\right]\right\|+\left\|\varphi \cdot \gamma_{p}^{(n-2)}\left(u^{n}(q)^{*}\right) u^{n}(p)^{*}-u^{n}(p q)^{*} \cdot \varphi\right\| \\
& \leq\left\|\gamma_{p}^{(n-2)}\left(u^{n}(q)^{*}\right)\left[u^{n}(p)^{*}, \varphi\right]\right\|+\left\|\left[\gamma_{p}^{(n-2)}\left(u^{n}(q)^{*}\right), \varphi\right] u^{n}(p)^{*}\right\| \\
& \quad \quad\left\|\varphi \cdot \gamma_{p}^{(n-2)}\left(u^{n}(q)^{*}\right) u^{n}(p)^{*}-u^{n}(p q)^{*} \cdot \varphi\right\| \\
& \leq 2 \varepsilon_{n-1}+\left\|\varphi \cdot \gamma_{p}^{(n-2)}\left(u^{n}(q)^{*}\right) u^{n}(p)^{*}-u^{n}(p q)^{*} \cdot \varphi\right\| \\
& =2 \varepsilon_{n-1}+\left\|c^{n-2}(p, q) \cdot \varphi \cdot \gamma_{p}^{(n-2)}\left(u^{n}(q)^{*}\right) u^{n}(p)^{*}-c^{n-2}(p, q) u^{n}(p q)^{*} \cdot \varphi\right\| \\
& \leq 2 \varepsilon_{n-1}+\left\|\left[\gamma_{p}^{(n-2)}\left(u^{n}(q)^{*}\right) u^{n}(p)^{*}, c^{n-2}(p, q) \cdot \varphi\right]\right\| \\
& \quad+\left\|\gamma_{p}^{(n-2)}\left(u^{n}(q)^{*}\right) u^{n}(p)^{*} c^{n-2}(p, q) \cdot \varphi-c^{n-2}(p, q) u^{n}(p q)^{*} \cdot \varphi\right\| \\
& \leq 2 \varepsilon_{n-1}+\left\|\gamma_{p}^{(n-2)}\left(u^{n}(q)^{*}\right)\left[u^{n}(p)^{*}, c^{n-2}(p, q) \cdot \varphi\right]\right\| \\
& \quad+\left\|\left[\gamma_{p}^{(n-2)}\left(u^{n}(q)^{*}\right), c^{n-2}(p, q) \cdot \varphi\right] u^{n}(p)^{*}\right\| \\
& \quad+\left\|\gamma_{p}^{(n-2)}\left(u^{n}(q)^{*}\right) u^{n}(p)^{*} c^{n-2}(p, q) \cdot \varphi-c^{n-2}(p, q) u^{n}(p q)^{*} \cdot \varphi\right\| \\
& \leq 4 \varepsilon_{n-1}+\left\|\gamma_{p}^{(n-2)}\left(u^{n}(q)^{*}\right) u^{n}(p)^{*} c^{n-2}(p, q) \cdot \varphi-c^{n-2}(p, q) u^{n}(p q)^{*} \cdot \varphi\right\| \\
& =4 \varepsilon_{n-1}+\left\|\left(u^{n}(p) \gamma_{p}^{(n-2)}\left(u^{n}(q)\right) c^{n-2}(p, q) u^{n}(p q)^{*}-c^{n-2}(p, q)\right) \cdot \varphi\right\| \\
& <5 \varepsilon_{n-1}
\end{aligned}
$$

holds for $p \in F_{n-1}, q \in S_{n-1}$ and $\varphi \in \Phi_{n-1}$.

By Lemma 4.9 , there exists a unitary $w_{n}$ such that

$$
\begin{aligned}
& \left\|\left(u^{n}(p) \gamma_{p}^{(n-2)}\left(w_{n}\right) w_{n}^{*}-1\right) \cdot \varphi\right\|<7 \sqrt[4]{\varepsilon_{n-1}}, \\
& \left\|\varphi \cdot\left(u^{n}(p) \gamma_{p}^{(n-2)}\left(w_{n}\right) w_{n}^{*}-1\right)\right\|<7 \sqrt[4]{\varepsilon_{n-1}}
\end{aligned}
$$

and

$$
\left\|\left[w_{n}, \psi\right]\right\|<\varepsilon_{n-1}
$$

for $\varphi \in \Phi_{n-1}, p \in F_{n-1}$ and $\psi \in \Psi_{n-1}^{\prime}$. Put $a^{n}(p)=u^{n}(p) \gamma_{p}^{(n-2)}\left(w_{n}\right) w_{n}^{*}$. We then obtain (n.8) and (n.9).

Set

$$
\begin{gathered}
b^{n}(p)=a^{n}(p) w_{n} b^{n-2}(p) w_{n}^{*}, \quad \theta_{n}=\operatorname{Ad}\left(w_{n}\right) \circ \theta_{n-2}, \\
c^{n}(p, q)=u^{n}(p) \gamma_{p}^{(n-2)}\left(u^{n}(q)\right) c^{n-2}(p, q) u^{n}(p q)^{*}, \\
\gamma_{p}^{(n)}=\operatorname{Ad}\left(u^{n}(p)\right) \circ \gamma_{p}^{(n-2)}=\operatorname{Ad}\left(a^{n}(p)\right) \circ \operatorname{Ad}\left(w_{n}\right) \circ \gamma_{p}^{(n-2)} \circ \operatorname{Ad}\left(w_{n}^{*}\right) .
\end{gathered}
$$

Then we obtain (n.3), (n.4) and (n.5). From (n.a) and (n.b), (n.6) and (n.7) follow. Thus the $n$-th step is complete, and we finished the induction. 
Lemma 5.3. The sequences of automorphisms $\left\{\theta_{2 n}\right\}_{n \in \mathbb{N}}$ and $\left\{\theta_{2 n+1}\right\}_{n \in \mathbb{N}}$ converge to some automorphisms.

Proof. Take $\psi \in \Psi_{n_{0}}$ for fixed $n_{0} \in \mathbb{N}$. If $n \geq n_{0}+1$, then $\psi, \theta_{n}(\psi) \in \Psi_{n+1}^{\prime}$. By $(n+2.9)$, we have

$$
\begin{aligned}
\left\|\theta_{n}(\psi)-\theta_{n+2}(\psi)\right\| & =\left\|\left[w_{n+2}, \theta_{n}(\psi)\right]\right\|<\varepsilon_{n+1}, \\
\left\|\theta_{n}^{-1}(\psi)-\theta_{n+2}^{-1}(\psi)\right\| & =\left\|\left[w_{n+2}, \psi\right]\right\|<\varepsilon_{n+1} .
\end{aligned}
$$

Since $\bigcup_{n} \Psi_{n}$ is total in $\mathcal{M}_{*},\left\{\theta_{2 n}^{ \pm 1}(\psi)\right\}_{n}$ and $\left\{\theta_{2 n+1}^{ \pm 1}(\psi)\right\}_{n}$ are Cauchy sequences for all $\psi \in \mathcal{M}_{*}$. Hence the limits $\bar{\theta}_{0}=\lim _{n} \theta_{2 n}$ and $\bar{\theta}_{1}=\lim _{n} \theta_{2 n+1}$ exist in the $u$-topology.

Lemma 5.4. The sequences of unitaries $\left\{b^{2 n}(p)\right\}_{n \in \mathbb{N}}$ and $\left\{b^{2 n+1}(p)\right\}_{n \in \mathbb{N}}$ converge to some unitaries in the $\sigma$-strong* topology.

Proof. Fix $p \in F_{n_{0}+1}$. Note that we have $\varphi_{0} \in \Phi_{m} \subset \Psi_{m}^{\prime}$ for all $m$, and $\varphi_{0} \cdot b^{n}(p)$, $b^{n}(p) \cdot \varphi_{0} \in \Psi_{n+1}^{\prime}, \operatorname{Ad}\left(b^{n}(p)\right)\left(\varphi_{0}\right) \in \Phi_{n+1}$ for $n \geq n_{0}$. By $(n+2.8)$ and $(n+2.9)$, we have

$$
\begin{aligned}
\| \varphi_{0} & \cdot\left(b^{n+2}(p)-b^{n}(p)\right) \| \\
& =\left\|\varphi_{0} \cdot\left(a^{n+2}(p) w_{n+2} b^{n}(p) w_{n+2}^{*}-b^{n}(p)\right)\right\| \\
& =\left\|\varphi_{0} \cdot\left(a^{n+2}(p)-b^{n}(p) w_{n+2} b^{n}(p)^{*} w_{n+2}^{*}\right)\right\| \\
& \leq\left\|\varphi_{0} \cdot\left(a^{n+2}(p)-1\right)\right\|+\left\|\varphi_{0} \cdot\left(1-b^{n}(p) w_{n+2} b^{n}(p)^{*} w_{n+2}^{*}\right)\right\| \\
& \leq 7 \sqrt[4]{\varepsilon_{n+1}}+\left\|\varphi_{0} \cdot\left(w_{n+2} b^{n}(p)-b^{n}(p) w_{n+2}\right)\right\| \\
& \leq 7 \sqrt[4]{\varepsilon_{n+1}}+\left\|\varphi_{0} \cdot w_{n+2} b^{n}(p)-w_{n+2} \cdot \varphi_{0} \cdot b^{n}(p)\right\|+\left\|\left[w_{n+2}, \varphi_{0} \cdot b^{n}(p)\right]\right\| \\
& \leq 7 \sqrt[4]{\varepsilon_{n+1}}+2 \varepsilon_{n+1} .
\end{aligned}
$$

We next estimate $\left\|\left(b^{n+2}(p)-b^{n}(p)\right) \cdot \varphi_{0}\right\|$. By $(n+2.8)$, we have

$$
\begin{aligned}
& \left\|\left(b^{n+2}(p)-b^{n}(p)\right) \cdot \varphi_{0}\right\| \\
& \quad=\left\|\left(a^{n+2}(p) w_{n+2} b^{n}(p) w_{n}^{*}-b^{n}(p)\right) \cdot \varphi_{0}\right\| \\
& \quad \leq\left\|a^{n+2}(p)\left(w_{n+2} b^{n}(p) w_{n+2}^{*}-b^{n}(p)\right) \cdot \varphi_{0}\right\|+\left\|\left(a^{n+2}(p)-1\right) b^{n}(p) \cdot \varphi_{0}\right\| \\
& \quad<\left\|\left(w_{n+2} b^{n}(p) w_{n+2}^{*}-b^{n}(p)\right) \cdot \varphi_{0}\right\|+7 \sqrt[4]{\varepsilon_{n+1}} .
\end{aligned}
$$

By $(n+2.9)$, the first term is estimated as follows:

$$
\begin{aligned}
& \left\|\left(w_{n+2} b^{n}(p) w_{n+2}^{*}-b^{n}(p)\right) \cdot \varphi_{0}\right\| \\
& \quad=\left\|\left(b^{n}(p) w_{n+2}^{*}-w_{n+2}^{*} b^{n}(p)\right) \cdot \varphi_{0}\right\| \\
& \quad \leq\left\|b^{n}(p) w_{n+2}^{*} \cdot \varphi_{0}-b^{n}(p) \cdot \varphi_{0} \cdot w_{n+2}^{*}\right\|+\left\|\left[w_{n+2}^{*}, b^{n}(p) \cdot \varphi_{0}\right]\right\|<2 \varepsilon_{n+1} .
\end{aligned}
$$

Hence we obtain

$$
\left\|\left(b^{n+2}(p)-b^{n}(p)\right) \cdot \varphi_{0}\right\|<7 \sqrt[4]{\varepsilon_{n+1}}+2 \varepsilon_{n+1} .
$$

The above estimation yields that $\hat{u}^{0}(p)=\lim _{n} b^{2 n}(p)$ and $\hat{u}^{1}(p)=\lim _{n} b^{2 n+1}(p)$ exist in the $\sigma$-strong* topology. 
The end of the proof of Theorem 5.1. By construction we have

$$
\gamma_{p}^{(2 n)}=\operatorname{Ad}\left(b^{2 n}(p)\right) \circ \theta_{2 n} \circ \alpha_{p} \circ \theta_{2 n}^{-1}
$$

and

$$
\gamma_{p}^{(2 n+1)}=\operatorname{Ad}\left(b^{2 n+1}(p)\right) \circ \theta_{2 n+1} \circ \beta_{p} \circ \theta_{2 n+1}^{-1} .
$$

Letting $n \rightarrow \infty$, we obtain

$$
\operatorname{Ad}\left(\hat{u}^{0}(p)\right) \circ \bar{\theta}_{0} \circ \alpha_{p} \circ \bar{\theta}_{0}^{-1}=\operatorname{Ad}\left(\hat{u}^{1}(p)\right) \circ \bar{\theta}_{1} \circ \beta_{p} \circ \bar{\theta}_{1}^{-1}
$$

by (n.6).

We will show the convergence of $\left\{c^{2 n}(p, q)\right\}_{n \in \mathbb{N}}$ and $\left\{c^{2 n+1}(p, q)\right\}_{n \in \mathbb{N}}$. Put

$$
\bar{w}_{2 n}=w_{2 n} w_{2 n-2} \cdots w_{2} \text {. }
$$

We can easily see $\theta_{2 n}=\operatorname{Ad}\left(\bar{w}_{2 n}\right)$ and $u^{2 n}(p) u^{2 n-2}(p) \cdots u^{2}(p)=b^{2 n}(p) \bar{w}_{2 n} \alpha_{p}\left(\bar{w}_{2 n}^{*}\right)$. Then it follows that

$$
\begin{aligned}
c^{2 n}(p, q)= & u^{2 n}(p) \gamma_{p}^{(2 n-2)}\left(u^{2 n}(q)\right) c^{2 n-2}(p, q) u^{2 n}(p q)^{*} \\
= & u^{2 n}(p) \gamma_{p}^{(2 n-2)}\left(u^{2 n}(q)\right) u^{2 n-2}(p) \gamma_{p}^{(2 n-4)}\left(u^{2 n-2}(q)\right) c^{2 n-4}(p, q) \\
& \times u^{2 n-2}(p q)^{*} u^{2 n}(p q)^{*} \\
= & u^{2 n}(p) u^{2 n-2}(p) \gamma_{p}^{(2 n-4)}\left(u^{2 n}(q) u^{2 n-2}(q)\right) c^{2 n-4}(p, q) u^{2 n-2}(p q)^{*} u^{2 n}(p q)^{*} .
\end{aligned}
$$

We repeat the above computation and obtain the following:

$$
\begin{aligned}
& c^{2 n}(p, q)= u^{2 n}(p) \cdots u^{2}(p) \alpha_{p}\left(u^{2 n}(q) \cdots u^{2}(q)\right) c^{a}(p, q) u^{2}(p q)^{*} \cdots u^{2 n}(p q)^{*} \\
&= b^{2 n}(p) \bar{w}_{2 n} \alpha_{p}\left(\bar{w}_{2 n}^{*}\right) \alpha_{p}\left(b^{2 n}(q) \bar{w}_{2 n} \alpha_{q}\left(\bar{w}_{2 n}^{*}\right)\right) c^{a}(p, q) \alpha_{p q}\left(\bar{w}_{2 n}\right) \bar{w}_{2 n}^{*} b^{2 n}(p q)^{*} \\
&= b^{2 n}(p) \bar{w}_{2 n} \alpha_{p}\left(\bar{w}_{2 n}^{*} b^{2 n}(q) \bar{w}_{2 n}\right) \alpha_{p} \alpha_{q}\left(\bar{w}_{2 n}^{*}\right) c^{a}(p, q) \alpha_{p q}\left(\bar{w}_{2 n}\right) \bar{w}_{2 n}^{*} b^{2 n}(p q)^{*} \\
&= b^{2 n}(p) \theta_{2 n} \circ \alpha_{p} \circ \theta_{2 n}^{-1}\left(b^{2 n}(q)\right) \bar{w}_{2 n} c^{a}(p, q) \alpha_{p q} \\
& \circ \sigma_{m(p, q)}\left(\bar{w}_{2 n}^{*}\right) \alpha_{p q}\left(\bar{w}_{2 n}\right) \bar{w}_{2 n}^{*} b^{2 n}(p q)^{*} \\
&= b^{2 n}(p) \theta_{2 n} \circ \alpha_{p} \circ \theta_{2 n}^{-1}\left(b^{2 n}(q)\right) \theta_{2 n}\left(c^{a}(p, q)\right) \bar{w}_{2 n} \\
& \quad \times \alpha_{p q}\left(\sigma_{m(p, q)}\left(\bar{w}_{2 n}^{*}\right) \bar{w}_{2 n}\right) \bar{w}_{2 n}^{*} b^{2 n}(p q)^{*} \\
&=b^{2 n}(p) \theta_{2 n} \circ \alpha_{p} \circ \theta_{2 n}^{-1}\left(b^{2 n}(q)\right) \theta_{2 n}\left(c^{a}(p, q)\right) \theta_{2 n} \circ \alpha_{p q} \\
& \quad \circ \theta_{2 n}^{-1}\left(\bar{w}_{2 n} \sigma_{m(p, q)}\left(\bar{w}_{2 n}^{*}\right)\right) b^{2 n}(p q)^{*} \\
&=b^{2 n}(p) \theta_{2 n} \circ \alpha_{p} \circ \theta_{2 n}^{-1}\left(b^{2 n}(q)\right) \theta_{2 n}\left(c^{a}(p, q)\right) \\
& \quad \times \theta_{2 n} \circ \alpha_{p q} \circ \theta_{2 n}^{-1}\left(\theta_{2 n}(\tilde{u}(m(p, q))) \tilde{u}(m(p, q))^{*}\right) b^{2 n}(p q)^{*} .
\end{aligned}
$$

Since the canonical extension is continuous in the $u$-topology, $\theta_{2 n}(\tilde{u}(m(p, q))) \tilde{u}(m(p, q))^{*}$ converges to $\bar{\theta}_{0}(\tilde{u}(m(p, q))) \tilde{u}(m(p, q))^{*}$. Similar results hold for $c^{2 n-1}(p, q)$. Then by $(n .7)$, we have

$$
\begin{gathered}
\hat{u}^{0}(p) \bar{\theta}_{0} \circ \alpha_{p} \circ \bar{\theta}_{0}^{-1}\left(\hat{u}^{0}(q)\right) \bar{\theta}_{0}\left(c^{\alpha}(p, q)\right) \bar{\theta}_{0} \circ \alpha_{p q} \circ \bar{\theta}_{0}^{-1}\left(\bar{\theta}_{0}(\tilde{u}(m(p, q))) \tilde{u}(m(p, q))^{*}\right) \hat{u}^{0}(p q)^{*} \\
=\hat{u}^{1}(p) \bar{\theta}_{1} \circ \beta_{p} \circ \bar{\theta}_{1}^{-1}\left(\hat{u}^{1}(q)\right) \bar{\theta}_{1} \circ \beta_{p q} \circ \bar{\theta}_{1}^{-1}\left(\bar{\theta}_{1}(\tilde{u}(m(p, q))) \tilde{u}(m(p, q))^{*}\right) \hat{u}^{1}(p q)^{*}
\end{gathered}
$$

and finished the proof of Theorem 5.1. 
5.2. Proof of the main theorem. In this subsection, we present a proof of our main result, Theorem 2.3, by using Theorem 5.1.

Proof of Theorem 2.3 (2). We use the same notation as in the previous subsection. Set

$$
\begin{gathered}
\bar{\theta}_{0}(\tilde{u}(n))=\tilde{u}^{\alpha}(n), \quad \bar{\theta}_{1}(\tilde{u}(n))=\tilde{u}^{\beta}(n), \quad n \in N, \\
\hat{u}(\tilde{p})=\hat{u}^{1}(p)^{*} \hat{u}^{0}(p), \quad \hat{u}_{n}=\tilde{u}^{\beta}(n) \tilde{u}^{\alpha}(n)^{*}, \quad p \in Q .
\end{gathered}
$$

We replace $\left(\alpha_{g}, a(g, h)\right)$ and $\left(\beta_{g}, 1\right)$ with $\left(\bar{\theta}_{0} \circ \alpha_{g} \circ \bar{\theta}_{0}^{-1}, \bar{\theta}_{0}(a(g, h))\right)$ and $\left(\bar{\theta}_{1} \circ \beta_{g} \circ \bar{\theta}_{1}^{-1}, 1\right)$ respectively. Note that we do not have $\alpha_{n}=\beta_{n}, n \in N$, after this replacement, and in fact we have

$$
\begin{aligned}
& \tilde{\alpha}_{n}=\bar{\theta}_{0} \circ \tilde{\sigma}_{n} \circ \bar{\theta}_{0}^{-1}=\bar{\theta}_{0} \circ \operatorname{Ad}(\tilde{u}(n)) \circ \bar{\theta}_{0}^{-1}=\operatorname{Ad}\left(\tilde{u}^{\alpha}(n)\right), \\
& \tilde{\beta}_{n}=\bar{\theta}_{1} \circ \tilde{\sigma}_{n} \circ \bar{\theta}_{1}^{-1}=\bar{\theta}_{1} \circ \operatorname{Ad}(\tilde{u}(n)) \circ \bar{\theta}_{1}^{-1}=\operatorname{Ad}\left(\tilde{u}^{\beta}(n)\right),
\end{aligned}
$$

and

$$
\beta_{n}=\operatorname{Ad}(\hat{u}(n)) \circ \alpha_{n}
$$

Claim. We have the following:

$$
\beta_{\tilde{p}}=\operatorname{Ad}(\hat{u}(\tilde{p})) \circ \alpha_{\tilde{p}}, \quad \hat{u}(\tilde{p}) \alpha_{\tilde{p}}(\hat{u}(\tilde{q})) c^{a}(p, q) \alpha_{\tilde{p q}}\left(\hat{u}_{m(p, q)}^{*}\right) \hat{u}(\widetilde{p q})^{*}=1, \quad p, q \in Q .
$$
we have

Indeed, $\beta_{\tilde{p}}=\operatorname{Ad}(\hat{u}(\tilde{p})) \circ \alpha_{\tilde{p}}$ is obvious. By the above replacement and Theorem 5.1,

$$
\begin{array}{r}
\hat{u}^{0}(\tilde{p}) \alpha_{\tilde{p}}\left(\hat{u}^{0}(\tilde{q})\right) c^{a}(p, q) \alpha_{\widetilde{p q}}\left(\tilde{u}^{\alpha}(m(p, q)) \tilde{u}(m(p, q))^{*}\right) \hat{u}^{0}(\widetilde{p q})^{*} \\
=\hat{u}^{1}(\tilde{p}) \beta_{\tilde{p}}\left(\hat{u}^{1}(\tilde{q})\right) \beta_{\widetilde{p q}}\left(\tilde{u}^{\beta}(m(p, q)) \tilde{u}(m(p, q))^{*}\right) \hat{u}^{1}(\widetilde{p q})^{*}
\end{array}
$$

Thus the following equalities hold:

$$
\begin{aligned}
1= & \beta_{\tilde{p}}\left(\hat{u}^{1}(\tilde{q})\right)^{*} \hat{u}^{1}(\tilde{p})^{*} \times \hat{u}^{0}(\tilde{p}) \alpha_{\tilde{p}}\left(\hat{u}^{0}(\tilde{q})\right) c^{a}(p, q) \alpha \widetilde{p q}\left(\tilde{u}^{\alpha}(m(p, q)) \tilde{u}(m(p, q))^{*}\right) \hat{u}^{0}(\widetilde{p q})^{*} \\
& \times \hat{u}^{1}(\widetilde{p q}) \beta \widetilde{p q}\left(\tilde{u}(m(p, q)) \tilde{u}^{\beta}(m(p, q))^{*}\right) \\
= & \beta_{\tilde{p}}\left(\hat{u}^{1}(\tilde{q})\right)^{*} \hat{u}(\tilde{p}) \alpha_{\tilde{p}}\left(\hat{u}^{0}(\tilde{q})\right) c^{a}(p, q) \alpha \widetilde{p q}\left(\tilde{u}^{\alpha}(m(p, q)) \tilde{u}(m(p, q))^{*}\right) \hat{u}(\widetilde{p q})^{*} \\
& \times \beta \widetilde{p q}\left(\tilde{u}(m(p, q)) \tilde{u}^{\beta}(m(p, q))^{*}\right) \\
= & \hat{u}(\tilde{p}) \alpha_{\tilde{p}}\left(\hat{u}^{1}(\tilde{q})\right)^{*} \alpha_{\tilde{p}}\left(\hat{u}^{0}(\tilde{q})\right) c^{a}(p, q) \alpha_{\widetilde{p q}}\left(\tilde{u}^{\alpha}(m(p, q)) \tilde{u}(m(p, q))^{*}\right) \\
& \quad \times \alpha_{\widetilde{p q}}\left(\tilde{u}(m(p, q)) \tilde{u}^{\beta}(m(p, q))^{*}\right) \hat{u}(\widetilde{p q})^{*} \\
= & \hat{u}(\tilde{p}) \alpha_{\tilde{p}}(\hat{u}(\tilde{q})) c^{a}(p, q) \alpha_{\widetilde{p q}}\left(\hat{u}(m(p, q))^{*}\right) \hat{u}(\widetilde{p q})^{*} .
\end{aligned}
$$

We will combine these results. We extend $\hat{u}(\cdot)$ to $\operatorname{Map}_{0}(G, \mathcal{U}(\mathcal{M}))$ by

$$
\hat{u}(n \tilde{r})=\hat{u}(n) \alpha_{n}(\hat{u}(\tilde{r})) a(n, \tilde{r}), \quad r \in Q, n \in N .
$$

Then $\beta_{g}=\operatorname{Ad}\left(\hat{u}_{g}\right) \circ \alpha_{g}$ holds for every $g \in G$. Set $z(g, h):=\hat{u}(g) \alpha_{g}(\hat{u}(h)) a(g, h) \hat{u}(g h)^{*}$, which is a 2-cocycle for $\beta$. To complete the proof, we only have to show $z(g, h)=1$. 
Since

$$
\begin{aligned}
\hat{u}(n) \alpha_{n} & (\hat{u}(\tilde{r})) d(a)(n, \tilde{r})^{*} \alpha_{\tilde{r}}\left(\hat{u}\left(\tilde{r}^{-1} n \tilde{r}\right)^{*}\right) \hat{u}(\tilde{r})^{*} \\
& =\hat{u}(n) \tilde{u}^{\alpha}(n) \hat{u}(\tilde{r}) \tilde{u}^{\alpha}(n)^{*} d(a)(n, \tilde{r})^{*} \alpha_{\tilde{r}}\left(\hat{u}\left(\tilde{r}^{-1} n \tilde{r}\right)^{*}\right) \hat{u}(\tilde{r})^{*} \\
& =\hat{u}_{n} \tilde{u}^{\alpha}(n) \tilde{\beta}_{\tilde{r}} \tilde{\alpha}_{\tilde{r}}^{-1}\left(\tilde{u}^{\alpha}(n)^{*} d(a)(n, \tilde{r})^{*} \alpha_{\tilde{r}}\left(\hat{u}\left(\tilde{r}^{-1} n \tilde{r}\right)^{*}\right)\right) \\
& =\lambda(n, \tilde{r}) \hat{u}(n) \tilde{u}^{\alpha}(n) \tilde{\beta}_{\tilde{r}}\left(\tilde{u}^{\alpha}\left(\tilde{r}^{-1} n \tilde{r}\right)^{*} \hat{u}\left(\tilde{r}^{-1} n \tilde{r}\right)^{*}\right) \\
& =\lambda(n, \tilde{r}) \tilde{u}^{\beta}(n) \tilde{\beta}_{\tilde{r}}\left(\tilde{u}^{\beta}\left(\tilde{r}^{-1} n \tilde{r}\right)^{*}\right)=1,
\end{aligned}
$$

we have $\hat{u}(n) \alpha_{n}(\hat{u}(\tilde{r})) a(n, \tilde{r})=\hat{u}(\tilde{r}) \alpha_{\tilde{r}}\left(\hat{u}\left(\tilde{r}^{-1} n \tilde{r}\right)\right) a\left(\tilde{r}, \tilde{r}^{-1} n \tilde{r}\right)$. Compare this result with Lemma 4.1.

Then in a similar way as in the proofs of Lemma 4.2 and Lemma 4.7, we can show $z(g, h)=1$ for $g, h \in G$. For the sake of completeness, we repeat the arguments presented in the proofs of Lemma 4.2 and Lemma 4.7.

By the definition of $u(n \tilde{p})$, we have

$$
z(n, \tilde{q})=\hat{u}(n) \alpha_{n}(\hat{u}(\tilde{q})) a(n, \tilde{q}) \hat{u}(n \tilde{q})^{*}=1 .
$$

Since

$$
\hat{u}(\tilde{p} m)=\hat{u}\left(\tilde{p} m \tilde{p}^{-1} \tilde{p}\right)=\hat{u}(\tilde{p}) \alpha_{\tilde{p}}(\hat{u}(m)) a(\tilde{p}, m)
$$

by the above result,

$$
z(\tilde{p}, m)=\hat{u}(\tilde{p}) \alpha_{\tilde{p}}(\hat{u}(m)) a(\tilde{p}, m) \hat{u}(\tilde{p} m)^{*}=1 .
$$

By using the 2-cocycle identity, we get

$$
z(m, n \tilde{q})=\beta_{m}\left(z(n, \tilde{q})^{*}\right) z(m, n) z(m n, \tilde{q})=1 .
$$

In a similar way, $z(\tilde{p} m, n)=1$ can be shown. Hence $z(g, h)=1$ if either of $g$ or $h$ is in $N$.

From

$$
\begin{aligned}
1 & =\hat{u}(\tilde{p}) \alpha_{\tilde{p}}(\hat{u}(\tilde{q})) c^{a}(p, q) \alpha_{\widetilde{p q}}\left(\hat{u}(m(p, q))^{*}\right) \hat{u}(\widetilde{p q})^{*} \\
& =z(\tilde{p}, \tilde{q}) z(\widetilde{p q}, m(p, q))^{*}=z(\tilde{p}, \tilde{q}),
\end{aligned}
$$

we have $z(\tilde{p}, \tilde{q})=1$ for $p, q \in Q$. Again the 2-cocycle identity yields

$$
z(m \tilde{p}, \tilde{q})=z(m, \tilde{p})^{*} \beta_{m}(z(\tilde{p}, \tilde{q})) z(m, \tilde{p} \tilde{q})=1 .
$$

In a similar way, $z(g, \tilde{q} n)=1$ can be shown, and $z(g, h)=1$ holds for all $g, h \in G$. Thus $(\alpha, a)$ and $(\beta, 1)$ are strongly cocycle conjugate.

\section{Model actions}

The construction of model actions with given invariant is presented in $[20,40]$ by using groupoid theory. However the essential point of using groupoid theory is to construct a right inverse for the Connes-Takesaki module map. Thus it may be possible to construct model actions without groupoid theory once one admits the existence of a right inverse of the module map [42]. In this section, we present the construction of model actions along this observation. 
6.1. Model action associated with a dominant weight. Let $\mathcal{M}$ be an injective factor of type III, $\varphi$ a dominant weight on $\mathcal{M}$, and $\mathcal{M}=\mathcal{M}_{\varphi} \rtimes_{\theta} \mathbb{R}$ the continuous decomposition of $\mathcal{M}$, and $u(s)$ the implementing unitary for $\theta$. Let $G$ be a discrete amenable group, and $\alpha$ an action of $G$ on $\mathcal{M}$. We quickly review how to describe $\operatorname{Inv}(\alpha)$ in terms of $\mathcal{M}_{\varphi}$.

We may assume that $\varphi \circ \alpha=\varphi$, and $\alpha_{g}(u(s))=u(s)$ by cocycle perturbation [41]. In this case, $\mathcal{M}_{\varphi}$ is invariant under $\alpha_{g}$, and we denote by $\alpha_{g}^{\prime}$ the restriction on $\mathcal{M}_{\varphi}$. Then $\operatorname{Inv}(\alpha)$ is obtained as follows. A Connes-Takesaki module is given by $\bmod \left(\alpha_{g}\right)=\left.\alpha_{g}^{\prime}\right|_{Z\left(\mathcal{M}_{\varphi}\right)}$. Let $N:=\left\{g \in G \mid \alpha_{g}^{\prime} \in \operatorname{Int}\left(\mathcal{M}_{\varphi}\right)\right\}$, which is a centrally trivial part of $\alpha$. Fix $v_{n} \in \mathcal{U}\left(\mathcal{M}_{\varphi}\right)$ with $\alpha_{n}^{\prime}=\operatorname{Ad}\left(v_{n}\right)$. Then we get a characteristic cocycle $(\lambda, \mu) \in Z\left(G, N, U\left(Z\left(\mathcal{M}_{\varphi}\right)\right)\right)$ and $c(n, t) \in Z^{1}\left(\mathbb{R}, \mathcal{U}\left(Z\left(\mathcal{M}_{\varphi}\right)\right)\right)$ as follows:

$$
\alpha_{g}^{\prime}\left(v_{g^{-1} n g}\right)=\lambda(n, g) v_{n}, \quad v_{m} v_{n}=\mu(m, n) v_{m n}, \quad \theta_{t}\left(v_{n}\right)=c(n, t) v_{n} .
$$

In this case, $\alpha_{n}=\operatorname{Ad}\left(v_{n}\right) \circ \sigma_{c(n)}^{\varphi}$ holds, where $\sigma_{c(n)}^{\varphi}$ is an extended modular automorphism [8]. Indeed, $\operatorname{Ad}\left(v_{n}^{*}\right) \circ \alpha_{n}=\mathrm{id}=\sigma_{c}^{\varphi}$ on $\mathcal{M}_{\varphi}$ by the choice of $v_{n}$, and

$$
\operatorname{Ad}\left(v_{n}^{*}\right) \circ \alpha_{n}(u(s))=v_{n}^{*} u(s) v_{n}=v_{n}^{*} \theta_{s}\left(v_{n}\right) u(s)=c(n, s) u(s)=\sigma_{c(n)}^{\varphi}(u(s))
$$

holds.

Conversely, for a normal subgroup $N \subset G$, a homomorphism

$$
\beta: g \in G / N \rightarrow \beta_{g} \in \operatorname{Aut}_{\theta}\left(Z\left(\mathcal{M}_{\varphi}\right)\right),
$$

$(\lambda, \mu)$, and $c(n, t)$, we will construct a model action $\gamma_{g}$ with $\operatorname{Inv}(\gamma)=\left(N, \beta_{g},[\lambda, \mu, c]\right)$.

By [42, Corollary 1.3], the exact sequence

$$
1 \longrightarrow \overline{\operatorname{Int}}(\mathcal{M}) \longrightarrow \operatorname{Aut}(\mathcal{M}) \stackrel{\bmod }{\longrightarrow} \operatorname{Aut}_{\theta}\left(Z\left(\mathcal{M}_{\varphi}\right)\right) \longrightarrow 1
$$

is split. By regarding $\beta$ as a faithful homomorphism from $G / \operatorname{Ker}(\beta)$ into $\operatorname{Aut}_{\theta}\left(Z\left(\mathcal{M}_{\varphi}\right)\right)$, we lift $\beta$ as an action of $G$ on $\mathcal{M}$ by the above splitting exact sequence. (Note $N \subset \operatorname{Ker}(\beta)$ ). We may assume $\varphi \circ \beta_{g}=\varphi, \beta_{g}(u(s))=u(s)$.

Let $\partial_{\theta}(u)(t):=u^{*} \theta_{t}(u) \in Z^{1}\left(\mathbb{R}, \mathcal{U}\left(Z\left(\mathcal{M}_{\varphi}\right)\right)\right)$ for $u \in U\left(Z\left(\mathcal{M}_{\varphi}\right)\right)$. Since

$$
c(m, t) c(n, t)=c(m n, t) \partial_{\theta}(\mu(m, n))(t), \quad \beta_{g}\left(c\left(g^{-1} n g, t\right)\right)=\partial_{\theta}(\lambda(n, g))(t) c(n, t),
$$

we have

$$
\sigma_{c(m)^{*}}^{\varphi} \circ \sigma_{c(n)^{*}}^{\varphi}=\sigma_{c(m)^{*} c(n)^{*}}^{\varphi}=\sigma_{\partial_{\theta}\left(\mu(m, n)^{*}\right) c(m n)^{*}}^{\varphi}=\operatorname{Ad}(\mu(m, n)) \circ \sigma_{c(m n)^{*}}^{\varphi},
$$

and

$$
\beta_{g} \circ \sigma_{c\left(g^{-1} n g\right)^{*}}^{\varphi} \circ \beta_{g}^{-1}=\sigma_{\beta_{g}\left(c\left(g^{-1} n g\right)^{*}\right)}^{\varphi}=\sigma_{\partial_{\theta}\left(\lambda(n, g)^{*}\right) c(n)^{*}}^{\varphi}=\operatorname{Ad}(\lambda(n, g)) \circ \sigma_{c(n)^{*}}^{\varphi} .
$$

In particular, $\left(\sigma_{c(n)^{*}}^{\varphi}, \mu(\cdot, \cdot)\right)$ is a cocycle crossed action of $N$.

Let $\mathcal{R}_{0}$ be the injective factor of type $\mathrm{II}_{1}$ with a tracial state $\tau$, and $\alpha_{g}^{(0)}$ a free action of $G$ on $\mathcal{R}_{0}$. Let $\alpha_{m}:=\sigma_{c(m)^{*}}^{\varphi} \otimes \alpha_{m}^{(0)}$, and take a twisted crossed product

$$
\mathcal{N}=\left(\mathcal{M} \otimes \mathcal{R}_{0}\right) \rtimes_{\alpha, \mu \otimes 1} N
$$


Let $v_{n}$ be the implementing unitary for $\alpha$. Since $\operatorname{Inv}(\alpha)$ is trivial, $\mathcal{N}$ and $\mathcal{M}$ have a common flow of weights by $[24,38]$. Hence $\mathcal{N} \cong \mathcal{M}$ by the classification theorem of injective factors. Let $\psi:=(\varphi \otimes \tau) \circ E$, where $E$ is the canonical conditional expectation on $\mathcal{M}$. Then $\psi$ is dominant, and $\mathcal{N}_{\psi}=\left(\mathcal{M}_{\varphi} \otimes \mathcal{R}_{0}\right) \otimes_{\alpha, \mu \otimes 1} N$.

Let $\gamma_{g}=\beta_{g} \otimes \alpha_{g}^{(0)}$. Since

$$
\begin{gathered}
\gamma_{g} \circ \alpha_{g^{-1} n g} \circ \gamma_{g}^{-1}=\operatorname{Ad}(\lambda(n, g) \otimes 1) \circ \alpha_{n}, \\
\lambda(m, g) \lambda(n, g) \mu(m, n) \lambda(m n, g)^{*}=\beta_{g}\left(\mu\left(g^{-1} m g, g^{-1} n g\right)\right), \\
\lambda(n, g h)=\beta_{g}\left(\lambda\left(g^{-1} n g, h\right)\right) \lambda(n, g),
\end{gathered}
$$

we can extend $\gamma$ to an action on $\mathcal{N}$ by $\gamma_{g}\left(v_{g^{-1} n g}\right)=(\lambda(n, g) \otimes 1) v_{n}$ by Theorem B.1 and Theorem B.2.

By the definition of $\gamma_{g}$, it is trivial that $\bmod \left(\gamma_{g}\right)=\beta_{g}$. If $m \in N$, then $\gamma_{m}^{\prime}=\left.\gamma_{m}\right|_{\mathcal{N}_{\psi}}$ is given by $\operatorname{Ad}\left(v_{m}\right)$. Indeed if $x \in \mathcal{M}_{\varphi} \otimes \mathcal{R}_{0}$, then

$$
\gamma_{m}^{\prime}(x)=\mathrm{id} \otimes \alpha_{m}^{(0)}(x)=\sigma_{c(m)^{*}}^{\varphi} \otimes \alpha_{m}^{(0)}(x)=\operatorname{Ad}\left(v_{m}\right)(x) .
$$

Note $\beta_{m}=$ id and $\left.\sigma_{c(m)^{*}}^{\varphi}\right|_{\mathcal{M}_{\varphi}}=$ id for $m \in N$. By

$$
\lambda(n, m)=\mu\left(m, m^{-1} n m\right) \mu(n, m)^{*}, \quad m, n \in N,
$$

we have

$$
\gamma_{m}^{\prime}\left(v_{m^{-1} n m}\right)=\lambda(n, m) v_{n}=\mu\left(m, m^{-1} n m\right) \mu(n, m)^{*} v_{n}=v_{m} v_{m^{-1} n m} v_{m}^{*} .
$$

Due to the freeness of $\alpha_{g}^{(0)}$, we have $\gamma_{g}^{\prime} \in \operatorname{Int}\left(\mathcal{N}_{\psi}\right)$ if and only if $g \in N$. By the definition of $N$ and $\gamma_{g}$, we have $\gamma_{g}\left(v_{g-1}{ }^{-1}\right)=\lambda(n, g) v_{n}$ and $v_{m} v_{n}=\mu(m, n) v_{m n}$. We can verify $\theta_{t} \otimes \mathrm{id}\left(v_{n}\right)=(c(n, t) \otimes 1) v_{n}$ as follows:

$$
\begin{aligned}
\theta_{t} \otimes \operatorname{id}\left(v_{n}\right) & =(u(t) \otimes 1) v_{n}(u(t) \otimes 1)^{*} \\
& =(u(t) \otimes 1) \alpha_{n}(u(-t) \otimes 1) v_{n} \\
& =\left(u(t) \sigma_{c(n)^{*}}^{\varphi}(u(-t)) \otimes 1\right) v_{n} \\
& =\left(u(t) c(n,-t)^{*} u(t) \otimes 1\right) v_{n} \\
& =\left(\theta_{t}\left(c(n,-t)^{*}\right) \otimes 1\right) v_{n}=(c(n, t) \otimes 1) v_{n} .
\end{aligned}
$$

The above argument shows that $\gamma$ realizes the given invariant.

Here we treat only type III factors, however this construction is valid for the type II case. If we use the results from [11], we can generalize the above construction for arbitrary faithful normal semifinite weights, which is the subject of the next subsection.

6.2. Generalization to arbitrary weight. In this subsection, we generalize the construction presented in the previous subsection by using [11].

Let $\mathfrak{W}(\mathcal{M})$ be the set of all faithful normal semifinite weights on $\mathcal{M}$. Let

$$
\partial_{\theta}: u \in \tilde{U}(\mathcal{M}) \rightarrow \partial_{\theta}(u)(t)=u^{*} \theta_{t}(u) \in Z_{\theta}^{1}(\mathbb{R}, \mathcal{U}(\mathcal{C}))
$$

be a coboundary map. By [11], for any faithful normal semifinite weight $\varphi \in \mathfrak{W}(\mathcal{M})$, there exists a homomorphism $b_{\varphi}: Z_{\theta}^{1}(\mathbb{R}, \mathcal{U}(\mathcal{C})) \rightarrow \tilde{U}(\mathcal{M})$ such that 
(1) $\partial_{\theta}\left(b_{\varphi}(c)^{*}\right)(t)=b_{\varphi}(c) \theta_{t}\left(b_{\varphi}(c)^{*}\right)=c(t)$,

(2) if $c(s)=e^{-i t s}$ for some $t \in \mathbb{R}$, then $b_{\varphi}(c)=\lambda^{\varphi}(t)^{*}\left(\right.$ hence $\left.\widetilde{\operatorname{Ad}}\left(b_{\varphi}(c)^{*}\right)=\sigma_{t}^{\varphi}\right)$,

(3) $b_{\varphi \circ \alpha}=\tilde{\alpha} \circ b_{\varphi} \circ \tilde{\alpha}^{-1}$,

(4) if $\varphi$ is dominant, then $\sigma_{c}^{\varphi}=\widetilde{\operatorname{Ad}}\left(b_{\varphi}(c)^{*}\right)$.

Namely $b_{\varphi}$ is a right section of the exact sequence

$$
1 \longrightarrow U(\mathcal{M}) \longrightarrow \tilde{U}(\mathcal{M}) \longrightarrow Z_{\theta}^{1}(\mathcal{U}(\mathcal{C})) \stackrel{\partial_{\theta}}{\longrightarrow} 1
$$

(The above exact sequence is a middle row of the characteristic square of $\mathcal{M}$.)

Thus we can define an extended modular automorphism $\sigma_{c}^{\varphi} \in \operatorname{Aut}(\mathcal{M})$ for arbitrary $\varphi \in \mathfrak{W}(\mathcal{M})$ by $\sigma_{c}^{\varphi}=\widetilde{\operatorname{Ad}}\left(b_{\varphi}(c)^{*}\right)$. (The condition (4) above justifies this definition.) Define a generalized Connes-cocycle by $[D \varphi: D \psi]_{c}:=b_{\varphi}(c)^{*} b_{\psi}(c)$. Indeed, we have

$$
[D \varphi: D \psi]_{c} \in \tilde{\mathcal{M}}^{\theta}=\mathcal{M}, \quad \operatorname{Ad}\left([D \varphi: D \psi]_{c}\right) \circ \sigma_{c}^{\psi}=\sigma_{c}^{\varphi}
$$

by the property of $b_{\varphi}(c)$ and the definition.

For $z \in U(\mathcal{C})$, set $\hat{z}:=b_{\varphi}\left(\partial_{\theta}(z)\right) z$. It is easy to see that $\hat{z} \in \mathcal{M}, \hat{z}^{*}=\widehat{z^{*}}, \widehat{z w}=\hat{z} \hat{w}$ and $\sigma_{\partial_{\theta}(z)}^{\varphi}=\operatorname{Ad}\left(\hat{z}^{*}\right)$.

As a natural generalization of the previous subsection, we show the following theorem.

Theorem 6.1. Let a normal subgroup $N$, a homomorphism $\beta: G / N \rightarrow \operatorname{Aut}_{\theta}(\mathcal{C})$, and a characteristic invariant $[\lambda, \mu] \in \Lambda_{\beta}(\tilde{G}, N, \mathcal{U}(\mathcal{C}))$ be given. Then for each fixed $\varphi \in \mathfrak{W}(\mathcal{M})$, there exists an action $\gamma$ of $G$ with $\operatorname{Inv}(\gamma)=(N, \beta,[\lambda, \nu])$.

Proof. Suppose a normal subgroup $N \subset G$, an action $\beta_{g} \in \operatorname{Aut}_{\theta}(\mathcal{C})$ of $G / N$, and a characteristic cocycle $(\lambda, \mu) \in Z_{\beta}(\tilde{G}, N, \mathcal{U}(\mathcal{C}))$ are given. As in the previous subsection, we denote $c(n, t):=\lambda(n,(e, t))$, and $\lambda(n, g)=\lambda(n,(g, 0))$ for $g \in G, n \in N$ and $t \in \mathbb{R}$.

Set $\hat{\lambda}(n, g):=\widehat{\lambda(n, g)}$ and $\hat{\mu}(m, n):=\widehat{\mu(m, n)}$. From the relation between $\mu$ and $c$, it follows that

$$
\sigma_{c(m)^{*}}^{\varphi} \circ \sigma_{c(n)^{*}}^{\varphi}=\sigma_{c(m)^{*} c(n)^{*}}^{\varphi}=\sigma_{\partial_{\theta}\left(\mu(m, n)^{*}\right) c(m n)^{*}}^{\varphi}=\operatorname{Ad}(\hat{\mu}(m, n)) \circ \sigma_{c(m n)^{*}}^{\varphi} .
$$

Namely $\left(\sigma_{c(n)^{*}}^{\varphi}, \hat{\mu}(m, n)\right)$ is a cocycle crossed action of $N$ on $\mathcal{M}$.

Once we obtain $\left(\sigma_{c(n)^{*}}^{\varphi}, \hat{\mu}(m, n)\right)$, we can construct a model action $\gamma_{g}$ as in $\S 6.1$. We lift $\beta_{g} \in \operatorname{Aut}_{\theta}(\mathcal{C})$ to $\operatorname{Aut}(\mathcal{M})$ by [42] as before and regard it as an action of $G$.

Set $a(n, g)=\left[D \varphi \circ \beta_{g}^{-1}: D \varphi\right]_{\beta_{g}\left(c\left(g^{-1} n g\right)^{*}\right)}$. Then

$$
\begin{aligned}
\beta_{g} \circ \sigma_{c\left(g^{-1} n g\right)^{*}}^{\varphi} \circ \beta_{g}^{-1} & =\sigma_{\beta_{g}\left(c\left(g^{-1} n g\right)^{*}\right)}^{\varphi \circ \beta_{-1}^{-1}} \\
& =\operatorname{Ad}(a(n, g)) \circ \sigma_{\beta_{g}\left(c\left(g^{-1} n g\right)^{*}\right)}^{\varphi} \\
& =\operatorname{Ad}(a(n, g)) \circ \sigma_{\partial_{\theta}\left(\lambda(n, g)^{*}\right) c(n)^{*}}^{\varphi} \\
& =\operatorname{Ad}(a(n, g) \hat{\lambda}(n, g)) \circ \sigma_{c(n)^{*}}^{\varphi}
\end{aligned}
$$

Set

$$
\mathcal{N}(\varphi, \lambda, \mu):=\left(\mathcal{M} \otimes R_{0}\right) \rtimes_{\sigma_{c^{*}}^{\varphi} \otimes \alpha^{(0)}, \hat{\mu}} N
$$


By Theorem B.1, we can define an automorphism $\gamma_{g}^{\varphi, \lambda, \mu} \in \operatorname{Aut}(\mathcal{N}(\varphi, \lambda, \mu))$ as follows:

$$
\begin{aligned}
\gamma_{g}^{\varphi, \lambda, \mu}(x) & =\beta_{g} \otimes \alpha_{g}^{(0)}(x), \quad x \in \mathcal{M} \otimes \mathcal{R}_{0}, \\
\gamma_{g}^{\varphi, \lambda, \mu}\left(v_{g^{-1} n g}\right) & =(a(n, g) \hat{\lambda}(n, g) \otimes 1) v_{n}, \quad n \in N .
\end{aligned}
$$

We show that $\gamma^{\varphi, \lambda, \mu}$ realizes the given invariants. For simplicity we denote $\gamma^{\varphi, \lambda, \mu}$ and $\mathcal{N}(\varphi, \lambda, \mu)$ by $\gamma$ and $\mathcal{N}$, respectively. We identify $\tilde{\mathcal{N}}$ and $\left(\tilde{\mathcal{M}} \otimes \mathcal{R}_{0}\right) \rtimes N$ in the standard way. As in the previous subsection, $\mathcal{N}$ is isomorphic to $\mathcal{M}$, and it is trivial that $\bmod \left(\gamma_{g}\right)=\beta_{g}$ holds.

We next show $N_{\gamma}=N$. Assume that $g \in G \backslash N$ and $a \in \tilde{\mathcal{N}}$ satisfy $\tilde{\gamma}_{g}(x) a=a x$ for all $x \in \tilde{\mathcal{N}}$. Let $a=\sum_{n \in N} a_{n} v_{n}$. Then for $x \in \tilde{\mathcal{M}}$, we have $\tilde{\gamma}_{g}(x) a_{n}=a_{n} \tilde{\gamma}_{n}(x)$, and hence $\tilde{\gamma}_{g n^{-1}}(x) a_{n}=a_{n} x$. Since $g n^{-1} \neq e$, we get $a_{n}=0$ for every $n \in N$. Thus $\gamma_{g} \notin \operatorname{Cnt}_{r}(\mathcal{N})$.

For $n \in N$, we will see that the canonical extension is given by

$$
\tilde{\gamma}_{n}=\operatorname{Ad}\left(b_{\varphi}\left(c(n)^{*}\right) \otimes 1\right) v_{n} .
$$

For simplicity, we omit $\otimes 1$ and denote $b_{\varphi}(c(n)) \otimes 1$ as $b_{\varphi}(c(n))$. For $a \in \tilde{\mathcal{M}} \otimes R_{0}$,

$$
\begin{aligned}
\operatorname{Ad}\left(b_{\varphi}(c(n))^{*} v_{n}\right)(a) & =\operatorname{Ad}\left(b_{\varphi}\left(c(n)^{*}\right)\right)\left(\sigma_{c(n)^{*}}^{\varphi} \otimes \alpha_{n}^{(0)}(a)\right) \\
& =\operatorname{id} \otimes \alpha_{n}^{(0)}(a)=\gamma_{n}(a) .
\end{aligned}
$$

By the definition of $\sigma_{c}^{\varphi}$, the relation $\sigma_{c}^{\varphi}\left(b_{\varphi}(d)\right)=b_{\varphi}(d)$ holds for $d \in Z_{\theta}^{1}(\mathbb{R}, \mathcal{U}(\mathcal{C}))$. Thus we have

$$
v_{m} b_{\varphi}\left(c(n)^{*}\right) v_{m}^{*}=\sigma_{c(m)^{*}}^{\varphi}\left(b_{\varphi}(c(n))^{*}\right)=b_{\varphi}\left(c(n)^{*}\right)
$$

and

$$
\begin{aligned}
\operatorname{Ad}\left(b_{\varphi}(c(n))^{*} v_{n}\right)\left(v_{n^{-1} m n}\right) & =\operatorname{Ad}\left(b_{\varphi}(c(n))^{*}\right)\left(v_{n} v_{n^{-1} m n} v_{n}^{*}\right) \\
& =\hat{\mu}\left(n, n^{-1} m n\right) \operatorname{Ad}\left(b_{\varphi}(c(n))^{*}\right)\left(v_{m n} v_{n}^{*}\right) \\
& =\hat{\mu}\left(n, n^{-1} m n\right) \hat{\mu}(m, n)^{*} \operatorname{Ad}\left(b_{\varphi}(c(n))^{*}\right)\left(v_{m}\right) \\
& =\hat{\lambda}(m, n) v_{m}=\gamma_{n}\left(v_{n^{-1} m n}\right) .
\end{aligned}
$$

Note that $a(m, n)=1$ for $m, n \in N$. Thus the canonical extension is given by the above formula.

The following computation shows $\chi(\gamma)=[\lambda, \mu]$. For $m, n \in N$, we have

$$
\begin{aligned}
b_{\varphi}(c(m))^{*} v_{m} b_{\varphi}(c(n))^{*} v_{n} & =b_{\varphi}(c(m))^{*} b_{\varphi}(c(n))^{*} v_{m} v_{n} \\
& =b_{\varphi}\left(c(m)^{*} c(n)^{*}\right) v_{m} v_{n} \\
& =b_{\varphi}\left(\partial_{\theta}\left(\mu(m, n)^{*}\right) c(m n)^{*}\right) \hat{\mu}(m, n) v_{m n} \\
& =b_{\varphi}\left(c(m n)^{*}\right) b_{\varphi}\left(\partial_{\theta}\left(\mu(m, n)^{*}\right)\right) \hat{\mu}(m, n) v_{m n} \\
& =\mu(m, n) b_{\varphi}(c(m n))^{*} v_{m n} .
\end{aligned}
$$

For $g \in G$ and $n \in N$, we have

$$
\begin{aligned}
\tilde{\gamma}_{g}\left(b_{\varphi}\left(c\left(g^{-1} n g\right)\right)^{*} v_{g^{-1} n g}\right) & =\beta_{g} \circ b_{\varphi}\left(c\left(g^{-1} n g\right)\right)^{*} \gamma_{g}\left(v_{g-1} n g\right) \\
& =b_{\varphi \circ \beta_{g}^{-1}}\left(\beta_{g}\left(c\left(g^{-1} n g\right)\right)\right) a(n, g) \hat{\lambda}(n, g) v_{n}
\end{aligned}
$$




$$
\begin{aligned}
& =b_{\varphi}\left(\beta_{g}\left(c\left(g^{-1} n g\right)\right)\right)^{*} \hat{\lambda}(n, g) v_{n} \\
& =b_{\varphi}\left(c(n)^{*} \partial_{\theta}\left(\lambda(n, g)^{*}\right)\right) \hat{\lambda}(n, g) v_{n} \\
& =\lambda(n, g) b_{\varphi}\left(c(n)^{*}\right) v_{n} .
\end{aligned}
$$

For $t \in \mathbb{R}$ and $n \in N$, it is easy to see that $\theta_{t}\left(b_{\varphi}(c(n))^{*} v_{m}\right)=c(n, t) b_{\varphi}(c(n))^{*} v_{m}$. Thus we have $\chi(\gamma)=[\lambda, \mu]$, and hence $\operatorname{Inv}(\gamma)=(N, \beta,[\lambda, \mu])$.

Although $\gamma^{\varphi, \lambda, \mu}$ realizes the given characteristic invariant $[\lambda, \mu]$, the above construction depends on the choice of a representative of $[\lambda, \mu]$. If we change $(\lambda, \mu)$ within its equivalence class in the above construction, we have the following result.

Let $\left(\lambda^{\prime}, \mu^{\prime}\right) \in Z(\tilde{G}, N, U(\mathcal{C}))$ be a characteristic cocycle which is equivalent to $(\lambda, \mu)$. Thus there exists $z \in \operatorname{Map}_{0}(N, \mathcal{U}(\mathcal{C}))$ such that

$$
\left(\lambda^{\prime}, \mu^{\prime}\right)=\left(\lambda \partial^{1}(z), \mu \partial^{2}(z)\right) .
$$

By Theorem B.2, we have the following result.

Theorem 6.2. Two model actions $\gamma^{\varphi, \lambda, \mu}$ and $\gamma^{\varphi, \lambda^{\prime}, \mu^{\prime}}$ are conjugate.

Proof. Set $c^{\prime}(n, t):=\lambda(n,(e, t))$. Thus we have $c^{\prime}(n, t)=z(n)^{*} c(n, t) \theta_{t}(z(n))$. Then

$$
\sigma_{c^{\prime}(n)^{*}}^{\varphi}=\sigma_{c(n)^{*} \partial_{\theta}\left(z(n)^{*}\right)}^{\varphi}=\operatorname{Ad}(\hat{z}(n)) \circ \sigma_{c(n)^{*}}^{\varphi}
$$

and

$$
\hat{\mu}^{\prime}(m, n)=\hat{z}(m) \hat{z}(n) \hat{\mu}(m, n) \hat{z}(m n)^{*},
$$

that is, $\left(\sigma_{c^{\prime}(\cdot)^{*}}^{\varphi}, \hat{\mu^{\prime}}\right)$ is a unitary perturbation of $\left(\sigma_{c^{*}}^{\varphi}, \hat{\mu}\right)$ by $\hat{z}$. From the relations $\lambda^{\prime}=\lambda \partial^{1}(z)$ and $\bmod \left(\gamma_{g}\right)=\beta_{g}, g \in G$, one obtains

$$
\hat{\lambda}^{\prime}(n, g)=\gamma_{g}^{\varphi, \lambda, \mu}\left(\hat{z}\left(g^{-1} n g\right)\right) \lambda(n, g) \hat{z}(n)^{*} .
$$

Hence $\gamma^{\varphi, \lambda^{\prime}, \mu^{\prime}}$ and $\gamma^{\varphi, \lambda, \mu}$ are conjugate by Theorem B.2.

We close this section by discussing the relation between model actions associated with different weights. Take $\varphi, \psi \in \mathfrak{W}(\mathcal{M})$. To clarify the dependence of $\varphi$, we write $\hat{\mu}(m, n)$ as $\hat{\mu}_{\varphi}(m, n)$ and so on. It is easy to see that $\left(\sigma_{c(\cdot)^{*}}^{\psi}, \hat{\mu}_{\psi}\right)$ is a unitary perturbation of $\left(\sigma_{c(\cdot)^{*}}^{\varphi}, \hat{\mu}_{\varphi}\right)$

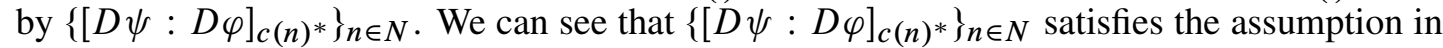
Theorem B.2. Thus the following holds.

Theorem 6.3. For any faithful normal semifinite weights $\varphi, \psi \in \mathfrak{W}(\mathcal{M})$, there exists an isomorphism $\theta_{\psi, \varphi}: \mathcal{N}(\varphi, \lambda, \mu) \rightarrow \mathcal{N}(\psi, \lambda, \mu)$ such that

$$
\theta_{\psi, \varphi} \circ \gamma_{g}^{\varphi, \lambda, \mu}=\gamma_{g}^{\psi, \lambda, \mu} \circ \theta_{\psi, \varphi}, \quad \theta_{\rho, \psi} \circ \theta_{\psi, \varphi}=\theta_{\rho, \varphi} .
$$

Proof. An isomorphism $\theta_{\psi, \varphi}$ is given as in Theorem B.2. The latter relation follows from the chain rule of generalized Connes-cocycles. 


\section{Group actions on subfactors}

In this section, we show that we can apply our previous argument for group actions on subfactors. We briefly recall basic notations for group actions of subfactors. See [9] for the basics of subfactor theory.

Let $\mathcal{N} \subset \mathcal{M}$ be a strongly amenable subfactor of type $\mathrm{II}_{1}$ in the sense of [34], and

$$
\mathcal{N} \subset \mathcal{M} \subset \mathcal{M}_{1}=\left\langle\mathcal{M}, e_{1}\right\rangle \subset \mathcal{M}_{2}=\left\langle\mathcal{M}_{1}, e_{2}\right\rangle \subset \cdots
$$

the Jones tower, where $e_{n}$ is the Jones projection for $\mathcal{M}_{n-1} \supset \mathcal{M}_{n-2}$. For $\alpha \in \operatorname{Aut}(\mathcal{M}, \mathcal{N})$, we extend $\alpha \in \operatorname{Aut}\left(\mathcal{M}_{n}\right)$ by $\alpha\left(e_{n}\right)=e_{n}$, and

$$
\Phi(\alpha)=\left\{\left.\alpha\right|_{\mathcal{M}^{\prime} \cap \mathcal{M}_{k}}\right\}_{k=0}^{\infty}
$$

denotes the Loi invariant [26]. An automorphism $\alpha$ is said to be strongly outer (see [1]) if $a \in \bigcup_{n} \mathcal{M}_{n}$ satisfying $\alpha(x) a=a x$ for all $x \in \mathcal{M}$ implies $a=0$.

Let $\mathrm{Cnt}_{r}(\mathcal{M}, \mathcal{N})$ be a set of all non-strongly outer automorphisms, and

$$
\chi_{a}(\mathcal{M}, \mathcal{N})=\left(\operatorname{Ker}(\Phi) \cap \operatorname{Cnt}_{r}(\mathcal{M}, \mathcal{N})\right) / \operatorname{Int}(\mathcal{M}, \mathcal{N})
$$

the algebraic $\chi$-group [12].

An automorphism $\alpha \in \operatorname{Aut}(\mathcal{M}, \mathcal{N})$ is approximately inner if $\alpha=\lim _{n} \operatorname{Ad}\left(u_{n}\right)$ in $\operatorname{Aut}(\mathcal{M})$ for $u_{n} \in U(\mathcal{N})$. An automorphism $\alpha$ is said to be centrally trivial if $\alpha=$ id on $\mathcal{M}^{\prime} \cap \mathcal{N}^{\omega}$. Here $\mathcal{M}^{\prime} \cap \mathcal{N}^{\omega}$ is a subfactor-version of a central sequence algebra. If $\mathcal{N} \subset \mathcal{M}$ is strongly amenable, then $\mathcal{M}^{\prime} \cap \mathcal{N}^{\omega}$ is of type $\mathrm{II}_{1}$. Denote the set of all approximately inner automorphisms and that of centrally trivial automorphisms by $\overline{\operatorname{Int}}(\mathcal{M}, \mathcal{N})$ and $\operatorname{Cnt}(\mathcal{M}, \mathcal{N})$, respectively.

To classify actions, we need the characterization of approximately inner automorphisms and centrally trivial automorphisms. Indeed for a strongly amenable subfactor $\mathcal{N} \subset \mathcal{M}$ of type $\mathrm{II}_{1}$, we have

$$
\operatorname{Ker}(\Phi)=\overline{\operatorname{Int}}(\mathcal{M}, \mathcal{N}), \quad \operatorname{Cnt}(\mathcal{M}, \mathcal{N})=\operatorname{Cnt}_{r}(\mathcal{M}, \mathcal{N}) .
$$

(See $[26,28,36]$ for a proof.)

Take $\alpha \in \operatorname{Ker}(\Phi)$ and $\sigma \in \operatorname{Cnt}_{r}(\mathcal{M}, \mathcal{N})$. Let $0 \neq a \in \mathcal{M}_{k}$ be an element such that $\sigma(x) a=a x$ holds for all $x \in \mathcal{M}$. Then there exists a unitary $u(\alpha, \sigma) \in \mathcal{N}$ such that $\alpha(a)=u(\alpha, \sigma) a$ (see [29]), which does neither depend on $a$ nor on $\mathcal{M}_{k}$. This $u(\alpha, \sigma)$ satisfies $\alpha \circ \sigma \circ \alpha^{-1}=\operatorname{Ad}(u(\alpha, \sigma)) \circ \sigma$. See [29] for more properties of $u(\alpha, \sigma)$.

We assume the following:

(1) The normalizer groups for $\mathcal{N} \subset \mathcal{M}$ and $\mathcal{M} \subset \mathcal{M}_{1}$ are trivial.

(2) There exists a lifting $\sigma: \chi_{a}(\mathcal{M}, \mathcal{N}) \rightarrow \operatorname{Aut}(\mathcal{M}, \mathcal{N})$.

Typical examples of such subfactors are the Jones subfactors with principal graph $A_{2 k+1}$, $k \geq 2$ (see [18]).

Let $(\alpha, a)$ be a cocycle crossed action of $G$ on $\mathcal{N} \subset \mathcal{M}$ with trivial Loi invariant. (This condition corresponds to the triviality of Connes-Takesaki modules in the previous sections.) Let

$$
N=\left\{h \in G \mid \alpha_{h} \in \operatorname{Cnt}(\mathcal{M}, \mathcal{N})\right\} .
$$


The $v$-invariant $v(n)$ is given by

$$
v(n)=\left[\alpha_{n}\right] \in \chi_{a}(\mathcal{M}, \mathcal{N}), \quad n \in N .
$$

Note that $v\left(g n g^{-1}\right)=v(n)$ holds by $\Phi\left(\alpha_{g}\right)=$ id. Let us fix a unitary $u_{n}^{\alpha} \in \mathcal{N}$ satisfying $\alpha_{n}=\operatorname{Ad}\left(u_{n}^{\alpha}\right) \circ \sigma_{v(n)}$. Then the characteristic invariant $[\lambda, \mu]$ for $\alpha$ is given as follows:

$$
\begin{gathered}
\alpha_{g}\left(u_{g^{-1} n g}^{\alpha}\right) u\left(\alpha_{g}, \sigma_{v(h)}\right)=\lambda(n, g) \delta a(g, n) u_{n}^{\alpha}, \\
u_{m}^{\alpha} \sigma_{\nu(m)}\left(u_{n}^{\alpha}\right)=\mu(m, n) a(m, n) u_{m n}^{\alpha} .
\end{gathered}
$$

We remark that $[\lambda, \mu]$ may be different from the usual characteristic invariant. Let

$$
\kappa(k, l):=u\left(\sigma_{l}, \sigma_{k}\right)^{*}
$$

be the $\kappa$-invariant for $\mathcal{N} \subset \mathcal{M}$, which becomes a bicharacter of $\chi_{a}(\mathcal{M}, \mathcal{N})$. (This notion comes from [16].) The only difference is the following relation:

$$
\lambda(n, m)=\mu\left(m, m^{-1} n m\right) \overline{\mu(n, m) \kappa(v(n), v(m))}, \quad m, n \in N .
$$

In [27, 29], we show that $\operatorname{Inv}(\alpha)=(N,[\lambda, \mu], v)$ is a complete cocycle conjugacy invariant for approximately inner actions of discrete amenable groups on subfactors under some restrictions, e.g., the triviality of the $\kappa$-invariant. However if we modify the argument in the previous sections in a suitable way, we can get rid of these restrictions.

Let $(\beta, b)$ be another cocycle crossed action of $G$ with $\operatorname{Inv}(\beta)=\operatorname{Inv}(\alpha)$. We choose $u_{m}^{\beta}$ which satisfies the same relation for $(\lambda, \mu)$. If we put $w_{n}:=u_{m}^{a} u_{m}^{\beta *}$ for $m \in N$, then

$$
\alpha_{n}=\operatorname{Ad}\left(w_{n}\right) \circ \beta_{n}, \quad w_{m} \alpha_{m}\left(w_{n}\right) a(m, n) w_{m n}^{*}=b(m, n)
$$

hold. Hence the same conclusion as in Lemma 3.1 holds. As in the single factor case, we may assume that $\alpha_{n}=\beta_{n}$ is a genuine action of $N$.

If $0 \neq b \in \mathcal{M}_{k}$ satisfies $\sigma_{\nu(n)}(x) b=b x$ for all $x \in \mathcal{M}$, then $b_{n}:=u_{n}^{\alpha} b$ satisfies $\alpha_{n}(x) b_{n}=b_{n} x$. Moreover, by the definition of $\lambda(n, g)$ and $\alpha_{g}\left(b_{n}\right)=u\left(\alpha_{g}, \sigma_{v(n)}\right) b_{n}$, we have

$$
\alpha_{g}\left(b_{g^{-1} n g}\right)=\lambda(n, g) d(a)(n, g) b_{n} .
$$

By using these facts, we can show a result similar to Lemma 4.1 as follows.

Lemma 7.1. For any $U_{g}=\left(u^{v}(g)\right) \in \mathcal{U}\left(\mathcal{N}^{\omega}\right)$ with $\lim _{v \rightarrow \omega} \alpha_{g}=\operatorname{Ad}\left(v_{g}^{v}\right) \circ \beta_{g}$, we have $U_{g}^{*} d(a)(n, g) \sigma_{n}\left(U_{g}\right)=d(b)(n, g)$.

Proof. Let $0 \neq b_{n} \in \mathcal{M}_{k}$ as above. Then

$$
\begin{aligned}
\lim _{\nu \rightarrow \omega} u^{\nu}(g)^{*} d(a)(n, g) \alpha_{n}\left(u^{\nu}(g)\right) b_{n} & =\lim _{\nu \rightarrow \omega} u^{\nu}(g)^{*} d(a)(n, g) b_{n} u^{\nu}(g) \\
& =\beta_{g} \alpha_{g}^{-1}\left(d(a)(n, g) b_{n}\right) \\
& =\lambda(n, g)^{*} \beta_{g}\left(b_{g^{-1} n g}\right) \\
& =d(b)(n, g) b_{n} .
\end{aligned}
$$

Let $E$ be the minimal conditional expectation from $\mathcal{M}_{k}$ to $\mathcal{M}$. Since $0 \neq b_{n} b_{n}^{*} \in \mathcal{M}^{\prime} \cap \mathcal{M}_{k}$, $0 \neq E\left(b_{n} b_{n}^{*}\right) \in \mathbb{C}$ follows, and hence we get the conclusion. 
Thus we can repeat the same argument as in the previous sections, and classify approximately inner actions of discrete amenable groups on $\mathcal{N} \subset \mathcal{M}$. In particular, the higher obstruction introduced in [22] and the $v$-invariant are complete outer conjugacy invariants for automorphisms on a Jones subfactor with principal graph $A_{2 k+1}, k \geq 2$. Note that all automorphisms of a Jones subfactor with principal graph $A_{n}, n \geq 4$, are approximately inner.

We close this section by explaining the construction of model actions.

Let $\mathcal{N} \subset \mathcal{M}$ be as above, and $\mathcal{R}_{0}$ the injective factor of type $\mathrm{II}_{1}$. Let $\alpha_{g}^{(0)}$ be a free action of $G$ on $\mathcal{R}_{0}$. Let

$$
\alpha_{n}:=\sigma_{v(n)^{-1}} \otimes \alpha_{n}^{(0)}, \quad \tilde{\mu}(m, n):=\mu(m, n) \kappa(v(m), v(n)) .
$$

Take a twisted crossed product

$$
\mathcal{A} \subset \mathcal{B}:=\left(\mathcal{N} \otimes \mathcal{R}_{0} \subset \mathcal{M} \otimes \mathcal{R}_{0}\right) \rtimes_{\alpha, \tilde{\mu}} N
$$

Since $\operatorname{Inv}(\alpha)$ is trivial, the standard invariant of $\mathcal{A} \subset \mathscr{B}$ and that of $\mathcal{N} \subset \mathcal{M}$ coincide, thus they are isomorphic by Popa's classification theorem [34]. Let $v_{n}$ be the implementing unitary, and define $\gamma_{g} \in \operatorname{Aut}(\mathscr{B}, \mathcal{A})$ as follows:

$$
\gamma_{g}(x)=\mathrm{id} \otimes \alpha_{g}^{(0)}(x), \quad x \in \mathcal{M} \otimes \mathcal{R}_{0}, \quad \gamma_{g}\left(v_{g^{-1} n g}\right)=\lambda(n, g) v_{n} .
$$

We extend $\sigma_{k} \otimes$ id to $\tilde{\sigma}_{k} \in \operatorname{Aut}(\mathscr{B}, \mathcal{A})$ by $\tilde{\sigma}_{k}\left(v_{n}\right)=\overline{\kappa(k, v(n))} v_{n}$. We claim that $\tilde{\sigma}_{k}$ is non-strongly outer. Take $0 \neq a \in \mathcal{M}_{k}$ such that $\sigma_{k}(x) a=a x, x \in \mathcal{M}$. It is clear that

$$
\tilde{\sigma}_{k}(x)(a \otimes 1)=(a \otimes 1) x
$$

for $x \in \mathcal{M} \otimes \mathcal{R}_{0}$. By the definition of the $\kappa$-invariant,

$$
\tilde{\sigma}_{k}\left(v_{n}\right)(a \otimes 1)=\overline{\kappa(k, v(n))} v_{n}(a \otimes 1)=\left(u\left(\sigma_{v(n)}, k\right) \sigma_{v(n)^{-1}}(a) \otimes 1\right) v_{n}=(a \otimes 1) v_{n} .
$$

Thus $\tilde{\sigma}_{k}$ is non-strongly outer.

We next show $\operatorname{Ad}\left(v_{n}\right) \circ \tilde{\sigma}_{n}=\gamma_{n}, n \in N$. For $x \in \mathcal{M} \otimes \mathcal{R}_{0}$, we have

$$
\operatorname{Ad}\left(v_{n}\right) \circ \tilde{\sigma}_{\nu(n)}=\operatorname{Ad}\left(v_{n}\right) \circ \sigma_{\nu(n)} \otimes \operatorname{id}(x)=\operatorname{id} \otimes \alpha_{n}^{0}(x)=\gamma_{n}(x) .
$$

If we use $\lambda(n, m)=\mu\left(m, m^{-1} n m\right) \overline{\mu(n, m) \kappa(\nu(n), v(m))}$, then

$$
\begin{aligned}
\operatorname{Ad}\left(v_{m}\right) \circ \tilde{\sigma}_{v(m)}\left(v_{m^{-1} n m}\right) & =\overline{\kappa(v(m), \mu(n))} v_{m} v_{m^{-1} n m} v_{m}^{*} \\
& =\overline{\kappa\left(v(m), \mu\left(m^{-1} n m\right)\right)} \tilde{\mu}\left(m, m^{-1} n m\right) \overline{\tilde{\mu}(n, m)} v_{n} \\
& =\mu\left(m, m^{-1} n m\right) \overline{\mu(n, m) \kappa(v(n), v(m))} v_{n} \\
& =\lambda(n, m) v_{n},
\end{aligned}
$$

and hence we have $\operatorname{Ad}\left(v_{n}\right) \tilde{\sigma}_{v(n)}=\gamma_{n}$. We can easily see that $\gamma_{g}$ is strongly outer for $g \in G \backslash N$. Since $\gamma_{g}(a \otimes 1)=a \otimes 1$, we have $u\left(\gamma_{g}, \tilde{\sigma}_{k}\right)=1$. By the definition of $\gamma$, it is trivial that $[\lambda, \mu]$ is a characteristic invariant for $\gamma$. 


\section{A. Proofs of Proposition 4.5 and Theorem 4.8}

In this appendix, we present the proofs of Proposition 4.5 and Theorem 4.8.

In [33, Proposition 7.4], Ocneanu proved the second cohomology vanishing theorem for semiliftable and strongly free actions of a discrete amenable group on $\mathcal{M}_{\omega}$ by means of the Rohlin theorem. However if we use the ultraproduct technique, we can present a simple proof of the second cohomology vanishing theorem for semiliftable cocycle crossed actions on a central sequence algebra without use of the Rohlin type theorem.

Lemma A.1. Let $R$ be a (not necessary separable) von Neumann algebra of type $\mathrm{II}_{1}$ with a tracial state $\tau, Q$ a discrete amenable group, and $(\alpha, u)$ a cocycle crossed action of $Q$ on $R$. Then for any finite subset $F \subset Q$ and $\varepsilon>0$, there exist unitaries $\{w(g)\}_{g \in Q} \subset R$ such that

$$
\left\|w(g) \alpha_{g}(w(h)) u(g, h) w(g h)^{*}-1\right\|_{1}<\varepsilon
$$

for $g, h \in F$.

Proof. Let $S$ be an $\left(F \cup F^{2}, \varepsilon / 2\right)$-invariant finite subset of $Q$. For $g \in Q$, we define a bijection $\ell(g)$ on $S$ so that $\ell(g) h=g h$ if $g h \in S$. Fix a system of matrix units $\left\{e_{g, h}\right\}_{g, h \in S} \subset R$ and set $N=\left\{e_{g, h}\right\}^{\prime} \cap R$. We identify $R$ with $N \otimes\left\{e_{g, h}\right\}^{\prime \prime}$. Fix a unitary $u(g)$ such that $\operatorname{Ad}(u(g)) \circ \alpha_{g}\left(e_{h, k}\right)=e_{h, k}$. By replacing $\{\alpha, u(g, h)\}$ with

$$
\left\{\operatorname{Ad}(u(g)) \circ \alpha_{g}, u(g) \alpha_{g}(u(h)) u(g, h) u(g h)^{*}\right\},
$$

we may assume $(\alpha, u(g, h))$ is of the form $\left(\alpha_{g} \otimes \mathrm{id}, u(g, h) \otimes 1\right)$ on $N \otimes\left\{e_{g, h}\right\}^{\prime \prime}$. Set

$$
w_{g}=\sum_{h \in S} u(g, h)^{*} \otimes e_{\ell}(g) h, h .
$$

For $g, h \in Q$, set $S_{g, h}=S \cap h^{-1} S \cap(g h)^{-1} S$. By the choice of $S$, we have $\left|S \backslash S_{g, h}\right| \leq \varepsilon|S| / 2, g, h \in F$.

Then we have

$$
\begin{aligned}
& w(g) \alpha_{g}(w(h))(u(g, h) \otimes 1) w(g h)^{*} \\
& =\left(\sum_{k \in S} u(g, k)^{*} \otimes e_{\ell(g) k, k}\right)\left(\sum_{l \in S} \alpha_{g}\left(u(h, l)^{*}\right) \otimes e_{\ell(h) l, l}\right) \\
& \quad \times(u(g, h) \otimes 1)\left(\sum_{m \in S} u(g h, m) \otimes e_{m, \ell(g h) m}\right) \\
& =\sum_{l \in S} u(g, \ell(h) l)^{*} \alpha_{g}\left(u(h, l)^{*}\right) u(g, h) u(g h, l) \otimes e_{\ell(g) \ell(h) l, \ell(g h) l} \\
& =\sum_{l \in S_{g, h}} 1 \otimes e_{g h l, g h l} \\
& \quad+\sum_{l \in S \backslash S_{g, h}} u(g, \ell(h) l)^{*} \alpha_{g}\left(u(h, l)^{*}\right) u(g, h) u(g h, l) \otimes e_{\ell(g) \ell(h) l, \ell(g h) l .} .
\end{aligned}
$$


Hence

$$
\begin{aligned}
& \left\|w(g) \alpha_{g}(w(h)) u(g, h) w(g h)^{*}-1\right\|_{1} \\
& \quad \leq \sum_{l \in S \backslash S_{g, h}}\left\|1 \otimes e_{l, l}\right\|_{1}+\sum_{l \in S \backslash S_{g, h}}\left\|1 \otimes e_{\ell}(g) \ell(h) l, \ell(g h) l\right\|_{1} \leq \varepsilon
\end{aligned}
$$

for $g, h \in F$.

Proof of Proposition 4.5. Let $F_{n}$ be a finite subset of $Q$ satisfying $F_{n} \subset F_{n+1}$ and $\bigcup_{n} F_{n}=Q$. Fix a faithful normal state $\phi_{0} \in \mathcal{M}_{*}$. Let $\left\{\Psi_{n}\right\}_{n=1}^{\omega}$ be an increasing sequence of finite sets such that $\bigcup_{n} \Psi_{n}$ is total in $\mathcal{M}_{*}$. Take representing sequences $\alpha_{g}=\left(\alpha_{g}^{v}\right) \in \operatorname{Aut}\left(\mathcal{M}_{\omega}\right)$ and $u(g, h)=\left(u(g, h)^{v}\right)_{v} \in \mathcal{U}\left(\mathcal{M}_{\omega}\right)$.

By Lemma A.1, for each $n$, there exist unitaries $\left\{w_{n}(g)\right\}_{g \in G} \subset \mathcal{U}\left(\mathcal{M}_{\omega}\right)$ such that

$$
\left\|w_{n}(g) \alpha_{g}\left(w_{n}(h)\right) u(g, h) w_{n}(g h)^{*}-1\right\|_{1}<\frac{1}{n}, \quad g, h \in F_{n} .
$$

Fix a representing sequence $w_{n}(g)=\left(w_{n}(g)^{v}\right)_{\nu}$. Take a neighborhood $W_{n}(\omega) \subset \mathbb{N}$ of $\omega$ such that

$$
\begin{aligned}
\phi_{0}\left(\left|w_{n}(g)^{v} \alpha_{g}^{v}\left(w_{n}(h)^{v}\right) u(g, h)^{v} w_{n}(g h)^{v *}-1\right|\right) & <\frac{1}{n}, \quad g, h \in F_{n}, \\
\phi_{0}\left(\left|w_{n}(g)^{v} \alpha_{g}^{v}\left(w_{n}(h)^{v}\right) u(g, h)^{v} w_{n}(g h)^{v *}-1\right|^{*}\right) & <\frac{1}{n}, \\
\left\|\left[w_{n}(g)^{v}, \psi\right]\right\| & <\frac{1}{n}, \quad \psi \in \Psi_{n}
\end{aligned}
$$

for $v \in W_{n}(\omega)$. We may assume $W_{n}(\omega) \supset W_{n+1}(\omega)$ and $\bigcap_{n} W_{n}(\omega)=\emptyset$.

Set $p(v)=n$ if $v \in W_{n}(\omega) \backslash W_{n+1}(\omega)$. Then $\{p(v)\}_{v}$ is an increasing sequence with $\lim _{v} p(v)=\infty$, and we have

$$
\begin{gathered}
\phi_{0}\left(\left|w_{p(v)}(g)^{v} \alpha_{g}^{v}\left(w_{p(v)}(h)^{v}\right) u(g, h)^{v} w_{p(v)}(g h)^{v *}-1\right|\right)<\frac{1}{p(v)}, \quad g, h \in F_{p(v)}, \\
\phi_{0}\left(\left|w_{p(v)}(g)^{v} \alpha_{g}^{v}\left(w_{p(v)}(h)^{v}\right) u(g, h)^{v} w_{p(v)}(g h)^{v *}-1\right|^{*}\right)<\frac{1}{p(v)}, \\
\left\|\left[w_{p(v)}(g)^{v}, \psi\right]\right\|<\frac{1}{p(v)}, \quad \psi \in \Psi_{p(v) .} .
\end{gathered}
$$

By the last inequality, $\left(w_{p(v)}(g)^{v}\right)_{\nu}$ is an $\omega$-centralizing sequence.

Set $w_{g}=\left(w_{p(v)}(g)^{v}\right)_{v} \in \mathcal{M}_{\omega}$. Then

$$
\left\|w(g) \alpha_{g}(w(h)) u(g, h) w(g h)^{*}-1\right\|_{1}=0
$$

holds, and hence $u(g, h)$ is a coboundary.

Next we present the proof of Theorem 4.8, which is a simplification of that of [33, Theorem 6.1]. Suppose that a countable subset $N \subset \mathcal{M}_{\omega}$ is given. We may assume $N$ is invariant under $\gamma$. Then $\gamma$ becomes a free proper action of $K$ on $N^{\prime} \cap \mathcal{M}_{\omega}$.

The following lemma due to Ocneanu [33, Lemma 6.3] is our starting point. 
Lemma A.2. Let $\varepsilon>0$ and $T \Subset K$ be such that $e \notin T$. Then there exists a partition of unity $\left\{e_{j}\right\}_{j=0}^{m} \subset N^{\prime} \cap \mathcal{M}_{\omega}$ such that

(1) $\left\|e_{0}\right\|_{1} \leq \varepsilon$,

(2) $e_{i} \gamma_{g}\left(e_{i}\right)=0,1 \leq i \leq m, g \in T$.

Take $F, S, \delta$ be as in Theorem 4.8. Let $E=\left\{E_{h}\right\}_{h \in S} \subset N^{\prime} \cap \mathcal{M}_{\omega}$ be a set of mutually orthogonal projections. For $g \in F$ we set

$$
\begin{gathered}
a_{g, E}=\sum_{h \in S \cap g^{-1} S}\left\|\gamma_{g}\left(E_{h}\right)-E_{g h}\right\|_{1}, \quad b_{E}=\sum_{h \in S}\left\|E_{h}\right\|_{1}, \\
c_{g, E}=\sum_{h \in S \backslash g^{-1} S}\left\|E_{h}\right\|_{1} .
\end{gathered}
$$

Lemma A.3. Let $E=\left\{E_{h}\right\}_{h \in S} \subset N^{\prime} \cap \mathcal{M}_{\omega}$ be a set of mutually orthogonal projections with $\left[\gamma_{g}\left(E_{h}\right), E_{k}\right]=0, g \in F, h, k \in S$. If $b_{E}<1-\delta^{\frac{1}{2}}$, then there exists a set of mutually orthogonal projections $E^{\prime}=\left\{E_{h}^{\prime}\right\}_{h \in S} \subset N^{\prime} \cap \mathcal{M}_{\omega}$ such that

(1) $0<2^{-1} \delta^{\frac{1}{2}} \sum_{h \in S}\left\|E_{h}^{\prime}-E_{h}\right\|_{1} \leq b_{E^{\prime}}-b_{E}$,

(2) $a_{g, E^{\prime}}-a_{g, E} \leq 2 \delta^{\frac{1}{2}}\left(b_{E^{\prime}}-b_{E}\right), g \in F$,

(3) $c_{g, E^{\prime}}-c_{g, E} \leq 2 \delta^{\frac{1}{2}}\left(b_{E^{\prime}}-b_{E}\right), g \in F$,

(4) $\left[\gamma_{g}\left(E_{h}^{\prime}\right), E_{k}^{\prime}\right]=0, g \in F, h, k \in S$.

Proof. Let us fix $\varepsilon>0$ such that $b_{E}<\left(1-\delta^{\frac{1}{2}}\right)(1-\varepsilon)$. By applying Lemma A.2 for $(F S)^{-1} F S \backslash\{e\}$, we get a partition of unity

$$
\left\{e_{j}\right\}_{j=0}^{m} \subset N^{\prime} \cap\left\{\gamma_{g}\left(E_{h}\right)\right\}_{g \in K, h \in S}^{\prime} \cap \mathcal{M}_{\omega}
$$

such that

$$
\left\|e_{0}\right\|_{1}<\varepsilon, \quad \gamma_{g}\left(e_{j}\right) \gamma_{h}\left(e_{j}\right)=0,
$$

for $1 \leq j \leq m, g, h \in F S, g \neq h$. Set

$$
x=|S|^{-1} \sum_{g, h \in S} \gamma_{g}^{-1}\left(E_{h}\right) .
$$

Then $\|x\|_{1}=\tau_{\omega}(x)=b_{E}$. Here we assume that

$$
\left\|e_{j} x\right\|_{1} \geq\left(1-\delta^{\frac{1}{2}}\right)\left\|e_{j}\right\|_{1}
$$

for all $1 \leq j \leq m$. Then

$$
\begin{aligned}
b_{E} & =\|x\|_{1} \geq\left\|\left(1-e_{0}\right) x\right\|_{1} \\
& =\sum_{j=1}^{m}\left\|e_{j} x\right\|_{1} \geq \sum_{j=1}^{m}\left(1-\delta^{\frac{1}{2}}\right)\left\|e_{j}\right\|_{1} \\
& =\left(1-\delta^{\frac{1}{2}}\right)\left\|1-e_{0}\right\|_{1} \geq\left(1-\delta^{\frac{1}{2}}\right)(1-\varepsilon)>b_{E}
\end{aligned}
$$


holds by the choice of $\varepsilon$. Hence we have $\left|e_{j} x\right|_{1}<\left(1-\delta^{\frac{1}{2}}\right)\left\|e_{j}\right\|_{1}$ for some $j$. Set $f=e_{j}$, $\tilde{f}=\sum_{g \in S} \gamma_{g}(f)$, and $\rho=\|\tilde{f}\|_{1}=|S|\|f\|_{1}$. Since $\|f x\|_{1}<\left(1-\delta^{\frac{1}{2}}\right)\|f\|_{1}$, we have

$$
\begin{aligned}
\left\|\tilde{f} \sum_{h \in S} E_{h}\right\|_{1} & =\sum_{g \in S}\left\|\gamma_{g}(f) \sum_{h \in S} E_{h}\right\|_{1} \\
& =\sum_{g \in S}\left\|f \sum_{h \in S} \gamma_{g}^{-1}\left(E_{h}\right)\right\|_{1} \\
& =|S|\|f x\|_{1}<|S|\left(1-\delta^{\frac{1}{2}}\right)\|f\|_{1} \leq\left(1-\delta^{\frac{1}{2}}\right) \rho .
\end{aligned}
$$

Set $f_{h}=\gamma_{h}(f)$, and $E_{h}^{\prime}=E_{h}(1-\tilde{f})+\gamma_{h}(f)$. We show $E_{h}^{\prime} \neq E_{h}$. If we assume $E_{h}^{\prime}=E_{h}$, then we get $\gamma_{h}(f)=E_{h} \tilde{f}$. However, this is impossible because we have

$$
\left(1-\delta^{\frac{1}{2}}\right) \rho>\sum_{h \in S}\left\|\tilde{f} E_{h}\right\|_{1}=\sum_{h \in S}\left\|\gamma_{h}(f)\right\|_{1}=\rho .
$$

Since $\left\{\gamma_{g}\left(f_{h}\right)\right\}$ are orthogonal projections for $g \in F, h \in S$, commuting with $\gamma_{g}\left(E_{h}\right)$ and $\left[\gamma_{g}\left(E_{h}\right), E_{k}\right]=0$, it follows that $\left[\gamma_{g}\left(E_{h}^{\prime}\right), E_{k}^{\prime}\right]=0$ for $g \in F, h, k \in S$. Thus the condition (4) follows.

We verify the condition (1). Since

$$
\sum_{h \in S}\left\|E_{h}^{\prime}-E_{h}\right\|_{1}=\sum_{h \in S}\left\|E_{h} \tilde{f}-f_{h}\right\|_{1} \leq\|\tilde{f}\|_{1}+\sum_{h \in S}\left\|f_{h}\right\|_{1} \leq 2 \rho
$$

and

$$
\begin{aligned}
b_{E^{\prime}} & =\sum_{h \in S}\left\|E_{h}^{\prime}\right\|_{1} \\
& =\sum_{h \in S}\left\|E_{h}(1-\tilde{f})+f_{h}\right\|_{1} \\
& =\sum_{h \in S}\left\|E_{h}\right\|_{1}+\sum_{h \in S}\left\|f_{h}\right\|_{1}-\sum_{h \in S}\left\|E_{h} \tilde{f}\right\|_{1} \\
& \geq b_{E}+\rho-\left(1-\delta^{\frac{1}{2}}\right) \rho=b_{E}+\delta^{\frac{1}{2}} \rho,
\end{aligned}
$$

we have

$$
b_{E}^{\prime}-b_{E} \geq \delta^{\frac{1}{2}} \rho \geq \frac{\delta^{\frac{1}{2}}}{2} \sum_{h \in S}\left\|E_{h}^{\prime}-E_{h}\right\|_{1},
$$

and we get condition (1).

We next verify condition (2). For $h \in S \cap g^{-1} S$,

$$
\begin{aligned}
\left\|\gamma_{g}\left(E_{h}^{\prime}\right)-E_{g h}^{\prime}\right\|_{1} & =\left\|\gamma_{g}\left(E_{h}(1-\tilde{f})+f_{h}\right)-E_{g h}(1-\tilde{f})-f_{g h}\right\|_{1} \\
& =\left\|\gamma_{g}\left(E_{h}(1-\tilde{f})\right)-E_{g h}(1-\tilde{f})\right\|_{1} \\
& \leq\left\|\gamma_{g}\left(E_{h}\right)(1-\tilde{f})-E_{g h}(1-\tilde{f})\right\|_{1}+\left\|\gamma_{g}\left(E_{h}\right)\left(\tilde{f}-\gamma_{g}(\tilde{f})\right)\right\|_{1} \\
& \leq\left\|\gamma_{g}\left(E_{h}\right)-E_{g h}\right\|_{1}+\left\|\gamma_{g}\left(E_{h}\right)\left(\tilde{f}-\gamma_{g}(\tilde{f})\right)\right\|_{1}
\end{aligned}
$$


holds. Hence we have

$$
\begin{aligned}
a_{g, E^{\prime}} & =\sum_{h \in S \cap g^{-1} S}\left\|\gamma_{g}\left(E_{h}^{\prime}\right)-E_{g h}^{\prime}\right\|_{1} \\
& \leq \sum_{h \in S \cap g^{-1} S}\left\|\gamma_{g}\left(E_{h}\right)-E_{g h}\right\|_{1}+\left\|\gamma_{g}\left(E_{h}\right)\left(\tilde{f}-\gamma_{g}(\tilde{f})\right)\right\|_{1} \\
& \leq a_{g, E}+\left\|\tilde{f}-\gamma_{g}(\tilde{f})\right\|_{1} .
\end{aligned}
$$

Here

$$
\begin{aligned}
\left\|\tilde{f}-\gamma_{g}(\tilde{f})\right\|_{1} & =\left\|\sum_{h \in S} \gamma_{h}(f)-\sum_{h \in S} \gamma_{g} \gamma_{h}(f)\right\|_{1} \\
& \leq \sum_{h \in S \triangle g S}\left\|\gamma_{h}(f)\right\|_{1} \leq 2 \delta|S|\|f\|_{1}=2 \delta \rho
\end{aligned}
$$

holds. These inequalities yield

$$
a_{g, E^{\prime}}-a_{g, E} \leq 2 \delta \rho \leq 2 \delta^{\frac{1}{2}}\left(b_{E^{\prime}}-b_{E}\right),
$$

and condition (2) holds.

We finally verify condition (3). Since we have

$$
\begin{aligned}
c_{g, E^{\prime}} & =\sum_{h \in S \backslash g^{-1} S}\left\|E_{h}^{\prime}\right\|_{1} \\
& =\sum_{h \in k \backslash g^{-1} S}\left\|E_{h}(1-\tilde{f})\right\|_{1}+\sum_{h \in S \backslash g^{-1} S}\left\|\gamma_{h}(f)\right\|_{1} \\
& \leq c_{g, E}+\left|S \backslash g^{-1} S\right|\|f\|_{1} \\
& \leq c_{g, E}+2 \delta|S|\|f\|_{1} \\
& \leq c_{g, E}+2 \delta \rho \\
& \leq c_{g, E}+2 \delta^{\frac{1}{2}}\left(b_{E^{\prime}}-b_{E}\right),
\end{aligned}
$$

we get condition (3).

Proof of Theorem 4.8. Let 8 be a set of all families of orthogonal projections $E=\left\{E_{h}\right\}_{h \in S}$ in $N^{\prime} \cap \mathcal{M}_{\omega}$ satisfying the following conditions.

(1) $a_{g, E} \leq 2 \delta^{\frac{1}{2}} b_{E}, g \in F$,

(2) $c_{g, E} \leq 2 \delta^{\frac{1}{2}} b_{E}, g \in F$,

(3) $\left[\gamma_{g}\left(E_{h}\right), E_{k}\right]=0, g \in F, h, k \in S$.

Obviously 8 is not empty. We define an order $E \leq E^{\prime}$ on 8 if $E=E^{\prime}$ or $E$ and $E^{\prime}$ satisfy the first three conditions in Lemma A.3. In the same way as in [33], it is shown that 8 is an inductive ordered set. Let $\bar{E}=\left\{\bar{E}_{h}\right\}$ be a maximal element, and assume $b_{\bar{E}}<1-\delta^{\frac{1}{2}}$. By Lemma A.3, there exists a partition of unity $\left\{E_{h}^{\prime}\right\}$ satisfying the conditions in Lemma A.3. 
Then it is easy to see that $\left\{E_{h}^{\prime}\right\}$ is in $\mathcal{S}$, and $\left\{E_{h}^{\prime}\right\}$ is strictly larger than $\bar{E}$. This contradicts the maximality of $\bar{E}$.

Hence $b_{\bar{E}} \geq 1-\delta^{\frac{1}{2}}$ holds. Let $E_{0}=1-\sum_{h \in S} \bar{E}_{h}$. Then $\left\|E_{0}\right\|_{1} \leq \delta^{\frac{1}{2}}$. Set

$$
E_{e}=\bar{E}_{e}+E_{0}
$$

and $E_{h}=\bar{E}_{h}$ for $h \neq e$. It is obvious that $\left[\gamma_{g}\left(E_{h}\right), E_{k}\right]=0, g \in F, h, k \in S$. It is also easy to see that $a_{g, E} \leq 4 \delta^{\frac{1}{2}}$ and $c_{g, E} \leq 3 \delta^{\frac{1}{2}}$.

\section{B. Extension of automorphisms to a twisted crossed product algebra}

Let $\mathcal{M}$ be a von Neumann algebra, and $L^{2}(\mathcal{M})$ the standard Hilbert space. Let $(\alpha, v)$ be a cocycle crossed action of a discrete group $G$. Define $\pi_{\alpha}(x), \lambda_{\alpha}(g) \in B\left(L^{2}(\mathcal{M}) \otimes \ell^{2}(G)\right)$, $x \in \mathcal{M}, g \in G$, as follows (do not confuse $\alpha_{g^{-1}}$ and $\alpha_{g}^{-1}$ ):

$$
\left(\pi_{\alpha}(x) \xi\right)(g)=\alpha_{g^{-1}}(x) \xi(g), \quad\left(\lambda_{\alpha}(g) \xi\right)(h)=v\left(h^{-1}, g\right) \xi\left(g^{-1} h\right) .
$$

Then one can easily verify that

$$
\lambda_{\alpha}(g) \pi_{\alpha}(x) \lambda_{\alpha}(g)^{*}=\pi_{\alpha}\left(\alpha_{g}(x)\right)
$$

and

$$
\lambda_{\alpha}(g) \lambda_{\alpha}(h)=\pi_{\alpha}(v(g, h)) \lambda_{\alpha}(g h)
$$

hold. By definition, a twisted crossed product von Neumann algebra is

$$
\mathcal{M} \rtimes_{\alpha, v} G=\pi_{\alpha}(\mathcal{M}) \vee\left\{\lambda_{\alpha}(g)\right\}_{g \in G} .
$$

Although the following result may be well known, we present a proof for the readers' convenience.

Theorem B.1. Let $(\alpha, v)$ be a cocycle crossed action of $G$ on $\mathcal{M}$.

(1) Assume that $\theta \in \operatorname{Aut}(\mathcal{M})$ induces an automorphism on $G$ (denoted by the same symbol $\theta$ ) such that

$$
\begin{gathered}
\theta \circ \alpha_{\theta^{-1}(g)} \circ \theta^{-1}=\operatorname{Ad}\left(u^{\theta}(g)\right) \circ \alpha_{g}(x), \\
u^{\theta}(g) \alpha_{g}\left(u^{\theta}(h)\right) v(g, h) u^{\theta}(g h)^{*}=\theta\left(v\left(\theta^{-1}(g), \theta^{-1}(h)\right)\right)
\end{gathered}
$$

for some unitary $u^{\theta}(g) \in \mathcal{M}$. Then there exists a unique extension $\tilde{\theta} \in \operatorname{Aut}\left(M \rtimes_{\alpha, v} G\right)$ of $\theta$ such that $\tilde{\theta}\left(\lambda\left(\theta^{-1}(g)\right)\right)=\pi\left(u^{\theta}(g)\right) \lambda(g)$.

(2) If we have $u^{\theta \sigma}(g)=\theta\left(u^{\sigma}\left(\theta^{-1}(g)\right)\right) u^{\theta}(g)$ for $\theta, \sigma \in \operatorname{Aut}(\mathcal{M})$, then $\tilde{\theta} \circ \tilde{\sigma}=\widetilde{\theta \circ \sigma}$ holds.

Proof. (1) For simplicity denote $\pi_{\alpha}, \lambda_{\alpha}(g)$, and $u^{\theta}(g)$ by $\pi, \lambda(g)$ and $u(g)$, respectively. Let $U_{\theta} \in B\left(L^{2}(\mathcal{M})\right)$ be the standard implementing unitary of $\theta$.

Define a unitary $W_{\theta} \in B\left(L^{2}(M) \otimes \ell^{2}(G)\right)$ by

$$
\left(W_{\theta} \xi\right)(g)=u\left(g^{-1}\right)^{*} U_{\theta} \xi\left(\theta^{-1}(g)\right), \quad \xi(g) \in L^{2}(M) \otimes \ell^{2}(G) .
$$


Then $W_{\theta}^{*}$ is given by

$$
\left(W_{\theta}^{*} \xi\right)\left(\theta^{-1}(g)\right)=U_{\theta}^{*} u\left(g^{-1}\right) \xi(g) .
$$

We show that $\operatorname{Ad} W_{\theta}$ gives a desired action. At first, we verify $\operatorname{Ad} W_{\theta}(\pi(a))=\pi(\theta(a))$ as follows:

$$
\begin{aligned}
\left(W_{\theta} \pi(a) W_{\theta}^{*} \xi\right)(g) & =u\left(g^{-1}\right)^{*} U_{\theta}\left(\pi(a) W_{\theta}^{*} \xi\right)\left(\theta^{-1}(g)\right) \\
& =u\left(g^{-1}\right)^{*} U_{\theta} \alpha_{\theta^{-1}\left(g^{-1}\right)}(a)\left(W_{\theta}^{*} \xi\right)\left(\theta^{-1}(g)\right) \\
& =u\left(g^{-1}\right)^{*} U_{\theta} \alpha_{\theta^{-1}\left(g^{-1}\right)}(a) U_{\theta}^{*} u\left(g^{-1}\right) \xi(g) \\
& =u\left(g^{-1}\right)^{*} \theta \alpha_{\theta^{-1}\left(g^{-1}\right)}(a) u\left(g^{-1}\right) \xi(g) \\
& =\alpha_{g^{-1}}(\theta(a)) \xi(g) \\
& =(\pi(\theta(a)) \xi)(g)
\end{aligned}
$$

Next we show that $\operatorname{Ad} W_{\theta}\left(\lambda\left(\theta^{-1}(g)\right)\right)=\pi(u(g)) \lambda(g)$ :

$$
\begin{aligned}
& \left(W_{\theta} \lambda\left(\theta^{-1}(g)\right) W_{\theta}^{*} \xi\right)(h) \\
& \quad=u\left(h^{-1}\right)^{*} U_{\theta}\left(\lambda(g) W_{\theta}^{*} \xi\right)\left(\theta^{-1}(h)\right) \\
& \quad=u\left(h^{-1}\right)^{*} U_{\theta} \alpha_{\theta^{-1}\left(h^{-1}\right)}\left(v\left(\theta^{-1}\left(h^{-1}\right), \theta^{-1}(g)\right)\right)\left(W_{\theta}^{*} \xi\right)\left(\theta^{-1}\left(g^{-1} h\right)\right) \\
& \quad=u\left(h^{-1}\right)^{*} U_{\theta} \alpha_{\theta^{-1}\left(h^{-1}\right)}\left(v\left(\theta^{-1}\left(h^{-1}\right), \theta^{-1}(g)\right)\right) U_{\theta}^{*} u\left(h^{-1} g\right) \xi\left(g^{-1} h\right) \\
& \quad=u\left(h^{-1}\right)^{*} \theta \alpha_{\theta^{-1}\left(h^{-1}\right)}\left(v\left(\theta^{-1}\left(h^{-1}\right), \theta^{-1}(g)\right)\right) u\left(h^{-1} g\right) \xi\left(g^{-1} h\right) \\
& \quad=u\left(h^{-1}\right)^{*} u\left(h^{-1}\right) \alpha_{h^{-1}}(u(g)) v\left(h^{-1}, g\right) \xi\left(g^{-1} h\right) \\
& \quad=\alpha_{h^{-1}}(u(g))(\lambda(g) \xi)(h) \\
& =(\pi(u(g)) \lambda(g) \xi)(h) .
\end{aligned}
$$

This shows that $\tilde{\theta}=\operatorname{Ad} W_{\theta}$ preserves $M \rtimes_{\alpha, v} G$, and is a desired extension. The uniqueness of $\tilde{\theta}$ is obvious.

(2) By the assumption, it is clear that $W_{\theta} W_{\sigma}=W_{\theta \sigma}$ holds, and hence $\widetilde{\theta \sigma}=\tilde{\theta} \tilde{\sigma}$ follows.

Let $(\beta, w)$ be a cocycle perturbation of $(\alpha, v)$ by $c \in \operatorname{Map}_{0}(G, \mathcal{U}(\mathcal{M}))$. It is well known that the crossed product algebras $M \rtimes_{\alpha, v} G$ and $M \rtimes_{\beta, w} G$ are isomorphic. In fact, we can construct a desired isomorphism as follows. Define $V \in L^{2}(\mathcal{M}) \otimes \ell^{2}(G)$ by

$$
(V \xi)(g)=c\left(g^{-1}\right) \xi(g) .
$$

Then $V$ is a unitary, and we have

$$
\operatorname{Ad}(V)\left(\pi_{\alpha}(a)\right)=\pi_{\beta}(a), \quad \operatorname{Ad}(V)\left(\lambda_{\alpha}(g)\right)=\pi_{\beta}\left(c(g)^{*}\right) \lambda_{\beta}(g) .
$$

Thus $\operatorname{Ad}(V)$ gives an isomorphism between $M \rtimes_{\alpha, v} G$ and $M \rtimes_{\beta, w} G$.

Assume there exists the same relation between $(\beta, w)$ and $\theta$ as in Theorem B.1. To clarify $\alpha$ and $\beta$, we denote by $u_{\alpha}(g)$ and $u_{\beta}(g)$ corresponding unitaries.

Theorem B.2. Let $\tilde{\theta}^{\alpha}$ and $\tilde{\theta}^{\beta}$ be the extension of $\theta$ to $M \rtimes_{\alpha, v} G$ and $M \rtimes_{\beta, w} G$ given in Theorem B.1, respectively. Assume that $u_{\beta}(g)=\theta\left(c\left(\theta^{-1}(g)\right)\right) u_{\alpha}(g) c(g)^{*}$. Then

$$
\operatorname{Ad}\left(V^{*}\right) \circ \tilde{\theta}^{\beta} \circ \operatorname{Ad}(V)=\tilde{\theta}^{\alpha}
$$

holds, and hence $\tilde{\theta}^{\alpha}$ and $\tilde{\theta}^{\beta}$ are conjugate. 
Proof. Let $W_{\theta}^{\alpha}$ and $W_{\theta}^{\beta}$ be unitaries constructed in the proof of Theorem B.1. If we show $V^{*} W_{\theta}^{\beta} V=W_{\theta}^{\alpha}$, then $\operatorname{Ad}\left(V^{*}\right) \circ \tilde{\theta}^{\beta} \circ \operatorname{Ad}(V)=\tilde{\theta}^{\alpha}$ follows. This is verified as follows:

$$
\begin{aligned}
\left(V^{*} W_{\theta}^{\beta} V \xi\right)(g) & =c\left(g^{-1}\right)^{*}\left(W_{\theta}^{\beta} V \xi\right)(g) \\
& =c\left(g^{-1}\right)^{*} u_{\beta}\left(g^{-1}\right)^{*} U_{\theta}(V \xi)\left(\theta^{-1}(g)\right) \\
& =c\left(g^{-1}\right)^{*} u_{\beta}\left(g^{-1}\right)^{*} U_{\theta} c\left(\theta^{-1}\left(g^{-1}\right)\right) \xi(g) \\
& =c\left(g^{-1}\right)^{*} u_{\beta}\left(g^{-1}\right)^{*} \theta\left(c\left(\theta^{-1}\left(g^{-1}\right)\right)\right) U_{\theta} \xi(g) \\
& =u_{\alpha}\left(g^{-1}\right)^{*} U_{\theta} \xi(g)=\left(W_{\theta}^{\alpha} \xi\right)(g) .
\end{aligned}
$$

\section{References}

[1] M. Choda and H. Kosaki, Strongly outer actions for an inclusion of factors, J. Funct. Anal. 122 (1994), 315332.

[2] A. Connes, Almost periodic states and factors of type $\mathrm{III}_{1}$, J. Funct. Anal. 16 (1974), 415-445.

[3] A. Connes, Outer conjugacy classes of automorphisms of factors, Ann. Sci. Eco. Norm. Sup. 8 (1975), 383419.

[4] A. Connes, Classification of injective factors, Ann. Math. 104 (1976), 73-115.

[5] A. Connes, On the classification of von Neumann algebras and their automorphisms, Symp. Math. XX (1976), 435-478.

[6] A. Connes, Periodic automorphisms of the hyperfinite factor of type $\mathrm{II}_{1}$, Acta Sci. Math. 39 (1977), 39-66.

[7] A. Connes, Factors of type $\mathrm{III}_{1}$, property $L_{\lambda}^{\prime}$ and closure of inner automorphisms, J. Operator Theory 14 (1985), 189-211.

[8] A. Connes and M. Takesaki, The flow of weights on factors of type III, Tohoku J. Math. 29 (1977), 473-555.

[9] D. E. Evans and Y. Kawahigashi, Quantum symmetries on operator algebras, Oxford Press, 1998.

[10] D. E. Evans and A. Kishimoto, Trace scaling automorphisms of certain stable AF algebras, Hokkaido Math. J. 26 (1997), 211-224.

[11] T. Falcone and M. Takesaki, The non-commutative flow of weights on a von Neumann algebra, J. Funct. Anal. 182 (2001), 170-206.

[12] S. Goto, Commutativity of automorphisms of subfactors modulo inner automorphisms, Proc. Amer. Math. Soc. 124 (1996), 3391-3398.

[13] U. Haagerup, Connes' bicentralizer problem and uniqueness of the injective factor of type III $_{1}$, Acta Math. 158 (1987), 95-148.

[14] U. Haagerup and E. Størmer, Equivalence of normal states on von Neumann algebras and the flow of weights, Adv. Math. 83 (1990), 180-262.

[15] J. Huebschmann, Group extensions, crossed pairs and an eight term exact sequence, J. reine angew. Math. 321 (1981), 150-172.

[16] V. F. R. Jones, A factor anti-isomorphic to itself but without involutory anti-automorphisms, Math. Scan. 46 (1980), 103-117.

[17] V. F. R. Jones, Actions of finite groups on the hyperfinite type $\mathrm{II}_{1}$ factor, Mem. Am. Math. Soc. 237 (1980).

[18] V. F. R. Jones, Index for subfactors, Invent. Math. 72 (1983), 1-25.

[19] V. F. R. Jones and M. Takesaki, Actions of compact abelian groups on semifinite injective factors, Acta Math. 153 (1984), 213-258.

[20] Y. Katayama, C. E. Sutherland and M. Takesaki, The characteristic square of a factor and the cocycle conjugacy of discrete group actions on factors, Invent. Math. 132 (1998), 331-380.

[21] Y. Katayama and M. Takesaki, Outer actions of a discrete amenable group on approximately finite dimensional factors, I, General theory, in: Operator algebras, quantization, and noncommutative geometry, Contemp. Math. 365, American Mathematical Society, Providence (2004), 181-237.

[22] Y. Kawahigashi, Classification of approximately inner automorphisms of subfactors, Math. Ann. 138 (1997), 425-438.

[23] Y. Kawahigashi, C. E. Sutherland and M. Takesaki, The structure of the automorphism group of an injective factor and the cocycle conjugacy of discrete abelian group actions, Acta Math. 169 (1992), 105-130.

[24] Y. Kawahigashi and M. Takesaki, Compact abelian group actions on injective factors, J. Funct. Anal. 105 (1992), 112-128. 
[25] W. Krieger, On ergodic flows and the isomorphism of factors, Math. Annal. 223 (1976), 19-70.

[26] P. Loi, On automorphisms of subfactors, J. Funct. Anal. 141 (1996), 275-293.

[27] T. Masuda, Notes on group actions on subfactors, J. Math. Soc. Japan 55 (2003), 1-11.

[28] T. Masuda, An analogue of Connes-Haagerup approach to classification of subfactors of type $\mathrm{III}_{1}$, J. Math. Soc. Japan 57 (2005), 959-1003.

[29] T. Masuda, Classification of approximately inner actions of discrete amenable groups on strongly amenable subfactors, Internat. J. Math. 16 (2005), 1193-1206.

[30] T. Masuda, Evans-Kishimoto type argument for actions of discrete amenable groups on McDuff factors, Math. Scand. 101 (2007), 48-64.

[31] T. Masuda and R. Tomatsu, Classification of minimal actions of a compact Kac algebra with amenable dual, Comm. Math. Phys. 274 (2007), 487-551.

[32] D. McDuff, Central sequences and the hyperfinite factor, Proc. London. Math. Soc. 21 (1970), 443-461.

[33] A. Ocneanu, Action of discrete amenable groups on von Neumann algebras, Lecture Notes in Math. 1138, Springer, Berlin 1985.

[34] S. Popa, Classification of amenable subfactor of type II, Acta Math. 172 (1994), 163-255.

[35] S. Popa, Some rigidity results for non-commutative Bernoulli shifts, J. Funct. Anal. 230 (2006), 273-328.

[36] S. Popa, Classification of actions of discrete amenable groups on amenable subfactors of type II, Internat. J. Math. 21 (2010), 1663-1695.

[37] J. G. Ratcliffe, Crossed extensions, Trans. Amer. Math. Soc. 257 (1980), 73-89.

[38] Y. Sekine, Flow of weights of the crossed products of type III factors by discrete groups, Publ. Res. Inst. Math. Sci. 26 (1990), 655-666.

[39] C. E. Sutherland, Extensions of von Neumann algebras, II, Publ. Res. Inst. Math. Sci. 16 (1980), $135-174$.

[40] C. E. Sutherland and M. Takesaki, Actions of discrete amenable groups and groupoids on von Neumann algebras, Publ. Res. Inst. Math. Sci. 21 (1985), 1087-1120.

[41] C. E. Sutherland and M. Takesaki, Actions of discrete amenable groups on injective factors of type III $\lambda$, $\lambda \neq 1$, Pacific. J. Math. 137 (1989), 405-444.

[42] C. E. Sutherland and M. Takesaki, Right inverse of the module of approximately finite-dimensional factors of type III and approximately finite ergodic principal measured groupoids, in: Operator algebras and their applications, II (Waterloo 1994/1995), Fields Inst. Commun. 20, American Mathematical Society, Providence (1998), 149-159.

[43] M. Takesaki, Theory of operator algebras, I, II, III, Springer-Verlag, Berlin 2002.

Toshihiko Masuda, Graduate School of Mathematics, Kyushu University, 744, Motooka, Nishi-ku, Fukuoka, 819-0395, Japan

e-mail:masuda@math.kyushu-u.ac.jp

Eingegangen 27. Juli 2010, in revidierter Fassung 15. November 2011 